\title{
Aerodynamic Shape Optimization of a Box-Wing Regional Aircraft Based on the Reynolds-Averaged Navier-Stokes Equations
}

by

Timothy Chau

A thesis submitted in conformity with the requirements

for the degree of Master of Applied Science

Graduate Department of Aerospace Science and Engineering University of Toronto

(c) Copyright 2017 by Timothy Chau 


\author{
Abstract \\ Aerodynamic Shape Optimization of a Box-Wing Regional Aircraft Based on the \\ Reynolds-Averaged Navier-Stokes Equations \\ Timothy Chau \\ Master of Applied Science \\ Graduate Department of Aerospace Science and Engineering \\ University of Toronto
}

2017

The box wing is an unconventional aircraft configuration that has the potential to provide major savings in fuel consumption relative to the conventional cantilever wing. In order to further develop and evaluate this potential, high-fidelity aerodynamic shape optimization is applied to the aerodynamic design of a box wing and a cantilever wing, based on the Embraer E190 regional jet, with the latter serving as a performance baseline. The optimization framework consists of B-spline parameterization, free-form and axial deformation geometry control, an integrated mesh-movement scheme based on the theory of linear elasticity, a Newton-Krylov-Schur flow solver for the Reynolds-averaged Navier-Stokes equations, a gradient-based optimizer, and the discrete-adjoint method for gradient evaluation. Results indicate that a box-wing with a heightto-span ratio of 0.26 burns $7.61 \%$ less fuel at cruise than a conventional baseline of the same span and lift. Aerodynamic trends and trade-offs are investigated, and a weight sensitivity study is performed. 


\section{Acknowledgements}

First and foremost, I would like to thank my supervisor, Professor David Zingg. I cannot thank him enough for the opportunity to work on such a state-of-the-art problem, which has proven to be challenging, but incredibly rewarding. I also have him to thank for the invaluable knowledge and experience that I have gained during these past few years. His passion for computational aerodynamics and environmentally-friendly aircraft design is something to be envied, and will always serve as a pillar of inspiration.

Furthermore, I would like to thank my Research Assessment Committee, Professor Craig Steeves and Professor Prasanth Nair, for the guidance they have provided me during the early stages of my work. On that note, I must also give thanks to Professor Masayuki Yano for sharing his insight during the final stages of my thesis.

I would also like to thank the UTIAS community for creating a welcoming and fun work environment. To the computational aerodynamics group, thank you for all of the helpful feedback you have given me over the years, and for making my late nights in the lab palatable. In particular, I would like to thank Dr. Shahriar Khosravi for the many interesting discussions on aerodynamics and structures, and Dr. Thomas Reist for his invaluable technical guidance and discussions on aircraft design.

I would also like to thank my friends and family for their unwavering support. To my parents, thank you for always believing in me, and for all of the sacrifices you have made to allow me the opportunity to pursuit my dreams. To my brother, thank you for being a constant source of strength, and for motivating me to aspire to greater heights.

To Gregg Streuber and Michael Piotrowski, thank you for making this journey an enjoyable one. Cheers to another few years together. To my friend Kim Vuong, thank you for all of the support you have given me over the years.

Lastly, I would like to thank the University of Toronto, the Government of Ontario, and the Natural Sciences and Engineering Research Council of Canada for their financial support. 


\section{Contents}

List of Tables $\quad$ v

List of Figures $\quad$ vi

List of Symbols and Abbreviations viii

1 Introduction $\quad 1$

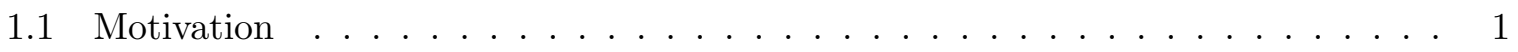

1.2 Aerodynamic Shape Optimization . . . . . . . . . . . . . . 5

1.3 The Box Wing . . . . . . . . . . . . . . . . . . 7

1.4 Thesis Objectives . . . . . . . . . . . . . . . . . . . . . . . 12

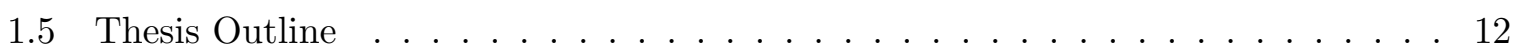

2 Methodology 13

2.1 Geometry Parameterization, Control, and Mesh Movement . . . . . . . . . . 13

2.1.1 B-Spline Parameterization . . . . . . . . . . . . . . . . 13

2.1.2 Grid Fitting and Grid Refinement . . . . . . . . . . . . . . 15

2.1.3 Free-Form and Axial Deformation Geometry Control . . . . . . . . . . . 16

2.1.4 Mesh Movement Scheme . . . . . . . . . . . . . . . . . . . . 19

2.2 Flow Solver . . . . . . . . . . . . . . . . . . 21

2.3 Gradient Evaluation and Optimization Algorithm . . . . . . . . . . . . . . . 22

2.3.1 The Optimization Problem . . . . . . . . . . . . . . . 22

2.3.2 Gradient Evaluation . . . . . . . . . . . . . . . 23

$2.3 .3 \mathrm{SNOPT} \ldots \ldots \ldots \ldots \ldots \ldots \ldots$

2.4 Geometry Definition . . . . . . . . . . . . . . . 25

2.5 Design Mission . . . . . . . . . . . . . . . . . 25

2.6 Weight and Balance . . . . . . . . . . . . . . . 26

3 Results $\quad 29$

3.1 The Cantilever-Wing Baseline . . . . . . . . . . . . . . . . . . . . . 29

3.1 .1 Problem Setup . . . . . . . . . . . . . . . . 29

3.1.2 Drag Minimization with Lift and Trim Constraints . . . . . . . . . . 33 
3.2 Exploratory Aerodynamic Shape Optimization of the Box Wing . . . . . . . . . 37

3.2 .1 Problem Setup . . . . . . . . . . . . . . . 37

3.2 .2 Lift-to-Drag Ratio Maximization . . . . . . . . . . . . . . . . . 42

3.2.3 Lift-to-Drag Ratio Maximization with a Trim Constraint . . . . . . . . . 44

3.3 Aerodynamic Design Optimization of the Box Wing . . . . . . . . . . . 50

3.3 .1 Problem Setup . . . . . . . . . . . . . . . . 50 50

3.3.2 Drag Minimization with Lift and Trim Constraints . . . . . . . . . . . 52

3.3.3 Weight Sensitivity Studies . . . . . . . . . . . . . . . 58

4 Euler- and RANS-Based Aerodynamic Shape Optimization Discussion 61

4.1 Problem Setup Comparison with Gagnon and Zingg . . . . . . . . . . . . 61

4.2 Results Comparison with Gagnon and Zingg . . . . . . . . . . . . . . . . . 62

5 Conclusions and Recommendations $\quad \mathbf{6 5}$

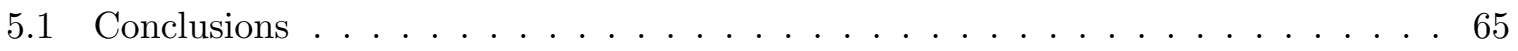

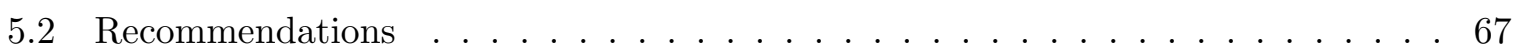

$\begin{array}{lr}\text { References } & 69\end{array}$ 


\section{List of Tables}

3.1 CTW100: Design summary. . . . . . . . . . . . . . . . 30

3.2 CTW100: Grid information. . . . . . . . . . . . . . 31

3.3 CTW100: Design variables and geometric constraints. . . . . . . . . . . . 33

3.4 CTW100: Grid convergence study. . . . . . . . . . . . . . . 37

3.5 CTW100: Optimization results. . . . . . . . . . . . . . . 37

3.6 BWE-1: Grid information. . . . . . . . . . . . . . . . . 40

3.7 BWE-1: Effective design variables and geometric constraints. . . . . . . . . . 43

3.8 BWE-2: Effective design variables and geometric constraints. . . . . . . . . . . 46

3.9 BW100: Design summary. . . . . . . . . . . . . . . . . 51

3.10 BW100: Grid information. . . . . . . . . . . . . . . . . . . 52

3.11 BW100: Design variables and geometric constraints. . . . . . . . . . . 53

3.12 BW100: Grid convergence study. . . . . . . . . . . . . . 58

3.13 BW100: Optimization results. . . . . . . . . . . . . . . . 58

3.14 Weight Sensitivity: Optimization results. . . . . . . . . . . . . . 60 


\section{List of Figures}

1.1 Circulation of a box wing based on linear aerodynamic theory, with the lift distribution over the fore and aft wings shown in blue and green, respectively, and the side-force distribution over the vertical tip fins shown in red. . . . . . 8

1.2 Relationship between induced drag and height-to-span ratio based on linear aerodynamic theory, relative to an optimum monoplane. . . . . . . . . . . . . . . . 9

2.1 A flowchart of Jetstream. . . . . . . . . . . . . . . . . . . . . . 14

2.2 The FFD geometry control system design variables for an airfoil. . . . . . . . . 18

2.3 A free-form and axial deformation geometry control system setup for a wing section which includes surface control points (red spheres), FFD volumes (black lines), FFD control points (blue spheres), axial curves (green lines), and axial curve control points (green spheres) . . . . . . . . . . . . . . 19

2.4 The control grid overlayed with the surface control points (red spheres) [left], and the corresponding fine grid $[$ right] for a cantilever wing. . . . . . . . . 21

3.1 CTW100: Initial planform. . . . . . . . . . . . . . . . 30

3.2 CTW100: Surface mesh and blocking topology. . . . . . . . . . . . . 31

3.3 CTW100: Free-form and axial deformation geometry control system for aerodynamic shape optimization which includes surface control points (red spheres), FFD volumes (black lines), FFD control points (blue spheres), axial curves (green lines), and axial curve control points (green spheres) . . . . . . . . . . 32

3.4 CTW100: Optimization history. The Merit function represents $C_{D}$. . . . . 34

3.5 CTW100: Surface pressure coefficient contours over the lower (left) and upper (right) surfaces of the optimized wing-tail geometry. The red sphere represents the CG. . . . . . . . . . . . . . . . . . . . . . 35

3.6 CTW100: Spanwise lift distribution. . . . . . . . . . . . 36

3.7 CTW100: Pressure coefficient distributions and airfoil profiles. . . . . . . . . 36

3.8 BWE-1: Initial planform. . . . . . . . . . . . . . . . . . 39

3.9 BWE-1: Surface mesh and blocking topology. . . . . . . . . . . . . . . . 39 
3.10 BWE-1: Free-form and axial deformation geometry control system for aerodynamic shape optimization which includes surface control points (red spheres), FFD volumes (black lines), FFD control points (blue spheres), axial curves (green lines), and axial curve control points (green spheres) [labeled]. . . . . . . . . . 41

3.11 BWE-1: Optimization history. The Merit function represents $L / D$. . . . . . . . 44

3.12 BWE-2: Optimization history. The Merit function represents $L / D$. . . . . . . 46

3.13 BWE-2: Surface pressure coefficient contours for the initial [left, (a)] and optimized [right, (a)] wing only geometries. Inboard and outboard views illustrate the signature closed-loop circulation pattern of the box wing. The CG of the optimized design is represented by the red sphere. . . . . . . . . . . . . . . 48

3.14 BWE-2: Force distributions over the fore wing (left), the vertical tip fin (middle), and the aft wing (right). . . . . . . . . . . . . . . . 49

3.15 BW100: Initial planform. . . . . . . . . . . . . . . . . 49

3.16 BW100: Free-form and axial deformation geometry control system for aerodynamic shape optimization which includes surface control points (red spheres), FFD volumes (black lines), FFD control points (blue spheres), axial curves (green lines), and axial curve control points (green spheres) . . . . . . . . . . . 51

3.17 BW100: Surface mesh and blocking topology. . . . . . . . . . . . . . 52

3.18 BW100: Optimization history. The Merit function represents $C_{D}$. . . . . . 54

3.19 BW100: Surface pressure coefficient contours over the lower (left) and upper (right) surfaces of the optimized wing geometry. The red sphere represents the

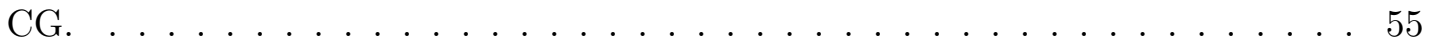

3.20 BW100: Pressure coefficient distributions and airfoil profiles for the fore wing of the box-wing aircraft configuration. . . . . . . . . . . . . . 56

3.21 BW100: Pressure coefficient distributions and airfoil profiles for the vertical tip fin of the box-wing aircraft configuration. For the optimized geometry, solid lines represent outboard surfaces, and dashed lines represent inboard surfaces. . . . . . 56

3.22 BW100: Pressure coefficient distributions and airfoil profiles for the aft wing of the box-wing aircraft configuration. . . . . . . . . . . . . . 57

3.23 BW100: Force distributions over the fore wing (left), the vertical tip fin (middle),

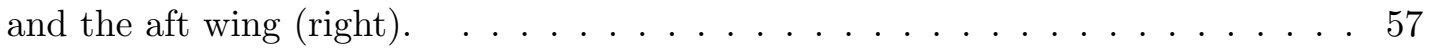

3.24 BW100: Plane cut of the normalized $z$-component of momentum located one MAC length downstream of the optimized wing geometry. . . . . . . . . . 58

3.25 Weight Sensitivity: Force distributions over the fore wing (left), the vertical tip fin (middle), and the aft wing (right). 


\section{List of Symbols and Abbreviations}

\section{Alphanumeric}

$\begin{array}{ll}\mathcal{A} & \text { Flow Jacobian } \\ \mathcal{C} & \text { Vector of general constraints } \\ \mathcal{F} & \text { FFD mapping function } \\ \mathcal{J} & \text { Objective function } \\ \mathcal{L} & \text { Lagrangian function } \\ \mathcal{M} & \text { Vector of mesh movement residuals } \\ \mathcal{N} & \text { B-spline basis function } \\ \mathcal{R} & \text { Vector of flow residuals } \\ \mathbf{A} & \text { B-spline surface control point coordinates } \\ a & \text { Speed of sound } \\ \mathbf{B} & \text { General B-spline control point coordinates } \\ \mathbf{b} & \text { B-spline volume control point coordinates } \\ b & \text { Span } \\ C_{D} & \text { Drag coefficient } \\ C_{D_{f}} & \text { Friction drag coefficient } \\ C_{D_{p}} & \text { Pressure drag coefficient } \\ C_{D_{\mathrm{F}}} & \text { Fuselage drag coefficient } \\ C_{D_{\mathrm{V}}} & \text { Vertical stabilizer drag coefficient } \\ C_{L} & \text { Lift coefficient } \\ C_{M} & \text { Pitching moment coefficient } \\ C_{P} & \text { Pressure coefficient } \\ c_{x z} & \text { Taper scaling factor for a given FFD-volume cross-section } \\ c_{z_{i}} & \text { Section shape scaling factor for FFD-volume control point } i \\ c_{T} & \text { Thrust specific fuel consumption } \\ \Delta t & \text { Time step } \\ e & \text { Foung's modulus for volume element } \varepsilon \\ E_{\varepsilon} & \\ F & F \\ \tilde{F} & \end{array}$




\begin{tabular}{|c|c|}
\hline f & Force vector \\
\hline$h / b$ & Height-to-span ratio \\
\hline $\mathbf{K}$ & Stiffness matrix \\
\hline$L / D$ & Lift-to-drag ratio \\
\hline$l / b$ & Stagger-to-span ratio \\
\hline$M$ & Mach number \\
\hline$m$ & Total mesh-movement increments \\
\hline$p$ & B-spline degree \\
\hline$\tilde{o}$ & Local origin \\
\hline q & Vector of mean conservative flow variables \\
\hline $\mathbf{R}$ & Rotation matrix operator \\
\hline$R$ & Range \\
\hline $\mathbf{S}$ & Scaling matrix operator \\
\hline$S$ & Reference or planform area \\
\hline$S_{\text {wet }}$ & Wetted area \\
\hline $\mathbf{T}$ & Translation matrix operator \\
\hline$T$ & B-spline knot vector \\
\hline $\mathbf{t}$ & Physical world space \\
\hline$\tilde{\mathbf{t}}$ & Deformed physical world space \\
\hline$u$ & Rotation axis or scaling direction vector \\
\hline $\mathbf{v}$ & Vector of design variables \\
\hline$V$ & Volume \\
\hline$V_{\varepsilon}$ & Mesh volume element \\
\hline $\boldsymbol{w}$ & Translation vector \\
\hline$W$ & Weight \\
\hline$x, y, z$ & Cartesian coordinates \\
\hline$x_{\mathrm{CG}}, z_{\mathrm{CG}}$ & Center of gravity coordinates \\
\hline $\bar{x}_{V}, \bar{z}_{V}$ & Volume centroid coordinates \\
\hline $\bar{x}_{\mathrm{AC}}, \bar{z}_{\mathrm{AC}}$ & Aircraft body center of gravity coordinates \\
\hline$y^{+}$ & Nondimensional off-wall distance \\
\hline
\end{tabular}

\section{Greek}

$\begin{array}{ll}\alpha & \text { Angle of attack } \\ \boldsymbol{\lambda}^{(i)} & \text { Mesh-adjoint variables at increment } i \\ \eta & \text { Normalized spanwise location or equivalent } \\ \Lambda & \text { Leading-edge sweep } \\ \Phi_{\varepsilon} & \text { Mesh orthogonality measure for volume element } \varepsilon \\ \boldsymbol{\psi} & \text { Flow-adjoint variables } \\ \theta & \text { Rotation angle }\end{array}$




$$
\boldsymbol{\xi}=\xi, \eta, \zeta \quad \text { Parametric coordinates }
$$

\section{Abbreviations}

$\begin{array}{ll}\text { AR } & \text { Aspect Ratio } \\ \text { BFGS } & \text { Broyden-Fletcher-Goldfarb-Shanno } \\ \text { CG } & \text { Center of Gravity } \\ \text { FFD } & \text { Free-Form Deformation } \\ \text { GMRES } & \text { Generalized Minimal Residual } \\ \text { GCROT } & \text { Generalized Conjugate Residual with Orthogonalization and Truncation } \\ \text { ILU } & \text { Incomplete Lower-Upper (Factorization) } \\ \text { KKT } & \text { Karush-Kuhn-Tucker } \\ \text { MAC } & \text { Mean Aerodynamic Chord } \\ \text { MTOW } & \text { Maximum Takeoff Weight } \\ \text { OEW } & \text { Operating Empty Weight } \\ \text { RANS } & \text { Reynolds-Averaged Navier-Stokes } \\ \text { SAT } & \text { Simultaneous Approximation Term } \\ \text { SBP } & \text { Summation-By-Parts } \\ \text { SNOPT } & \text { Sparse Nonlinear OPTimizer }\end{array}$




\section{Chapter 1}

\section{Introduction}

\subsection{Motivation}

In recent times, the impact of the aviation industry on the environment has become an increasingly pressing issue. Air travel has steadily grown at an average rate of $6 \%$ per year and represents approximately $2 \%$ of total anthropogenic carbon dioxide $\left(\mathrm{CO}_{2}\right)$ emissions [1]. To put this in perspective, the number of passengers worldwide increased by 170 million between 2013 and 2014 to a total of 3.3 billion passengers [1]. That year, air travel contributed more than 640 million tonnes of human-made $\mathrm{CO}_{2}$ emissions ${ }^{1}$ - a quantity that will only continue to rise for the foreseeable future. $\mathrm{CO}_{2}$ emissions are of particular concern given their long lifetimes, which are on the order of hundreds of years. Meanwhile, some non- $\mathrm{CO}_{2}$ emissions remain only for at most weeks at a time, but have strong short term effects on climate change. These emissions are harmful to the environment due to the altitudes at which they are injected into the atmosphere. For instance, at cruise altitudes, namely, in the upper troposphere or the lower stratosphere, chemical interactions between nitrogen oxides $\left(\mathrm{NO}_{x}\right)$ and the atmosphere can result in a net increase in radiative forcing or warming through the production of ozone [3, 4]. Other non- $\mathrm{CO}_{2}$ emissions include soot and water vapor which also contribute positive radiative forcing, whether directly, or indirectly as enablers for the formation of contrails and aviation-induced cirrus [3].

These trends have caused the global community to recognize the need for a more environmentally-friendly aviation industry. This recognition is perhaps best exemplified by the goals set forth by the International Air Transport Association (IATA), which are to improve aircraft fuel efficiency by $1.5 \%$ per year, to attain carbon neutral growth by 2020 , and to achieve a $50 \%$ reduction in aviation $\mathrm{CO}_{2}$ emissions by 2050, relative to those of 2005 [5]. Indeed, these goals are representative of efforts made around the world through programs such as the GARDN project in Canada [6], the Sustainable Aviation project in the United Kingdom [7], and NASA's Environmentally Responsible Aviation project [8]. Another major program is ACARE's Flightpath 2050, which intends to achieve reductions of $50 \%$ and $80 \%$ in

An approximation based on 2010 data released by the Intergovernmental Panel on Climate Change [2] 
$\mathrm{CO}_{2}$ and $\mathrm{NO}_{x}$ emissions, respectively, by 2020, relative to 2000, and reductions of $75 \%$ and $90 \%$ in $\mathrm{CO}_{2}$ and $\mathrm{NO}_{x}$ emissions, respectively, by 2050 [9]. Common among these programs is the general consensus that major advances in aircraft technologies, aircraft operations, and sustainable low-carbon fuels are necessary if these targets are to be met.

Today, the rate at which the demand for air travel is increasing is far exceeding that at which improvements in fuel economy are being attained. This trend is likely the result of the long turnaround involved in the research and development of new aircraft technologies, paired with the fact that airline fleets remain in service for up to 25 years before being phased out for more fuel-efficient designs. In the short term, improvements in aircraft operations can begin to suppress this offset. While on the ground, fuel can be conserved by reducing the time over which aircraft remain in idle through better aircraft management, and by implementing more fuel efficient aircraft taxiing procedures. While in the air, aircraft can ascend to efficient cruise conditions more quickly, and descend continuously for better performance. However, step changes in fuel economy must be realized if the future of the aviation industry is to remain environmentally sustainable.

In the long term, alternative fuels can offer major reductions in greenhouse gas emissions. Examples include biofuels such as biodiesel and synthetic kerosene, which have the benefit of reduced carbon signatures and more environmentally-friendly production cycles [3]. However, the viability of these alternatives, and others, is still being assessed. In particular, two questions remain: (1) can biofuels provide a net reduction in greenhouse gas emissions when accounting for the complete life-cycle, e.g. increased nitrous oxide $\left(\mathrm{N}_{2} \mathrm{O}\right)$ from agricultural soil, and (2) can a sufficient amount of feedstock be acquired, without the destructive use of arable land, to produce a practical supply of fuel, even for modest blend ratios? In the event that these concerns can be overcome, alternative fuels must still pass strict safety regulations and compatibility concerns with current and or future engine technologies [3].

In the interim, improvements to aircraft technologies will play a critical role in mitigating the impact of aviation on the environment. Areas of advancement include aircraft aerodynamics, aircraft structural weight, and engine efficiency. For an aircraft at steady level flight, the effect these disciplines have on fuel consumption can best be understood through the Breguet range equation, written in the form,

$$
W_{\text {fuel }}=W_{\text {final }}\left[\exp \left(\frac{c_{T} R}{a M(L / D)}\right)-1\right]
$$

where $W_{\text {final }}$ is the final aircraft weight at the end of cruise, $c_{T}$ is the thrust specific fuel consumption, $R$ is the cruise range, $a$ and $M$ are the speed of sound and the Mach number at cruise, respectively, and $L / D$ is the lift-to-drag ratio. This relationship indicates that for a fixed range, $R$, the quantity of fuel burned, $W_{\text {fuel }}$, depends directly on structural weight, $W_{\text {final }}$, engine efficiency, $c_{T}$, and aerodynamic performance, $L / D$.

In terms of engine efficiency, fuel consumption can be reduced in a number of ways. For example, an engine with a geared shaft system can allow the compressor and turbine to each 
operate at their optimum rotational speeds. In fact, this concept is already in service today on product lines like the PurePower series from Pratt and Whitney Canada [10]. Other examples include unducted fan engines [11], boundary-layer ingesting engines [12, 13], and the more distant electric propulsion system [14].

One example of reducing the structural weight of an aircraft is by employing lightweight materials such as composites. Once again, this concept is already in service today. For example, $50 \%$ of the Boeing 787 Dreamliner consists of composite materials, which provides an overall weight reduction of $20 \%$, relative to an equivalent noncomposite design [15]. Similarly, $53 \%$ of the Airbus A350 XWB is composed of composite materials, which contributes to its $25 \%$ improvement in fuel efficiency over its current long-range competitors [16].

With respect to aircraft aerodynamics (and in many cases, structures), perhaps the most promising idea is to explore unconventional aircraft configurations that have the potential to provide major savings in fuel consumption, relative to the conventional tube-and-wing design. Over the past half-century, a number of concepts have been proposed, each capitalizing on one or more aerodynamic benefits. Examples include the blended wing-body configuration [17], the lifting-fuselage configuration [18], the D8 [19], the strut-braced wing configuration [20], and the box-wing [21] and joined-wing configurations [22].

For the blended wing-body configuration, a preliminary design analysis has suggested that fuel consumption can be reduced by as much as $27 \%$, relative to an equivalent conventional baseline [17]. This advantage comes from having a streamlined surface that combines the wing and the fuselage, which not only reduces interference drag, but can also lead to a reduction in wetted area and therefore, friction drag. The blended wing-body also benefits from a lifting fuselage, which alleviates wing-bending loads and thus enables a lighter structure. These advantages, however, come at the cost of stability and control challenges, and a less efficient means for maintaining fuselage pressure loads - two benefits of the conventional tube-and-wing design. Other variations include the lifting-fuselage configuration, which takes on a more elongated wing-body design and performs better in the regional class segment [18].

The D8 is another unconventional aircraft configuration that features a lifting fuselage alongside a wing and tail. The fuselage is a wide "double-bubble" design that enables the benefit of reduced wing-bending loads while not straying too far from the efficient load bearing cylindrical structure of the conventional tube-and-wing design. What sets this fuselage apart from that of the blended wing-body and lifting-fuselage configurations is a prominent lifting nose, which provides a pitch-up moment to aid in trimming the aircraft. This leads to a reduction in downforce required by the horizontal stabilizer, and translates to a gain in aerodynamic efficiency.

The D8 is designed for short and medium range missions similar to the Boeing 737-800, and operates at lower Mach numbers [19]. In conjunction with the use of thinner wings offered by the lessened structural requirements from the lifting fuselage, flying at lower speeds eliminates the need for wings with high sweep angles. As a result, the D8 benefits from a further reduction 
in structural weight and is less susceptible to wave drag.

Other unconventional aircraft designs include the strut-braced wing configuration, and the box-wing and joined-wing configurations. These configurations can improve the aerodynamic performance of aircraft through merits of better wing design alone. Because of their compatibility with conventional fuselage and empennage designs, these aircraft configurations are of particular interest due to their potential for near-term implementation.

The primary advantage of the strut-braced wing configuration is a significant reduction in induced drag from having a larger span - a quality attributed to the inverse proportionality between induced drag and the square of the wing span. This improvement can be obtained without a penalty to weight by virtue of the strut, which provides support for the additional wing-bending loads $[23,24]$. In fact, the structural efficiency offered by the strut-braced system exceeds the minimum support required for the extended span and thus can be further leveraged to decrease the structural volume within the wing. This leaves open the possibility of decreasing the thickness-to-chord ratio for reducing wave drag [25]. With less wave drag comes less of a need for high sweep angles, which like for the D8, makes for an increased laminar flow extent over the wing and therefore a decrease in viscous drag. Given the structural freedom provided by the strut, alternative designs could opt to keep the same span for prioritizing a reduction in wing weight and or thickness.

The box-wing configuration also benefits from lower induced drag, but by virtue of being a nonplanar wing system. In fact, the box wing is considered to be superior to all other wing configurations for a given span, height, and lift [26]. For a box wing with a height-to-span ratio of 0.3 , classical lifting-line theory predicts that a $40 \%$ reduction in induced drag can be attained, relative to an equivalent cantilever-wing baseline. In theory, these benefits can be achieved without an increase in wetted area or weight, owing to the distribution of load between the fore and aft wings and the inherent increase in structural depth offered by the braced wing system [27]. A similar design is the joined wing, which opts to connect the forward and aft wings at the tips directly by discarding the vertical tip fins. In this way, height-to-span ratio, or aerodynamic efficiency, is sacrificed for an increase in structural stiffness [28].

Given their high potential for providing significant improvements to fuel economy, unconventional aircraft configurations have been acknowledged by green initiatives around the world as key mitigation measures against the impact of commercial aviation on the environment. However, this potential must be properly assessed if the industry is to be convinced to invest billions of U.S. dollars in their development - a challenge in and of itself given the gap in knowledge surrounding these novel concepts and the general unavailability of empirical data. Fortunately, advances in numerical methods for applications in high-fidelity aerodynamic shape optimization, paired with modern high performance computing power, have enabled in-depth studies of these radically new aircraft designs. 


\subsection{Aerodynamic Shape Optimization}

Numerical methods in aerodynamic shape optimization offer a means for automatically deforming an initial aircraft design into a form that optimizes performance as defined by the designer. These algorithms often comprise a flow solver, an optimizer, geometry and mesh definition, and a method for updating the geometry and mesh. This provides the user with a high level platform for designing fuel efficient aircraft that places less emphasis on a priori knowledge, thus facilitating the evaluation of unconventional aircraft configurations while leaving open the possibility of uncovering novel designs that exhibit superior aerodynamic performance.

In contrast to the typical "trial and error" approach, numerical methods in aerodynamic shape optimization are capable of finding the best possible design, by virtue of the optimizer. In general, at least in principle, this is true for gradient-free methods such as genetic algorithms [29], and in some instances, surrogate-assisted algorithms [30]. Genetic algorithms use ideas from evolutionary theory to find the optimum design. These methods are based on natural selection and use concepts such as reproduction and mutation to converge to the global optimum. Meanwhile, surrogate-assisted algorithms construct an approximation of the design space using a database of design points and use this information to thoroughly search the design space for the optimum solution. Despite their advantages, however, gradient-free methods are associated with large computational costs. This makes them impractical for problems in highfidelity aerodynamic shape optimization, where design spaces are often defined by hundreds of design variables.

As an alternative, gradient-based methods are relatively inexpensive when compared to the computational cost of gradient-free methods. Gradient-based methods follow a path defined by the gradient of the objective function ${ }^{2}$ and offer fast convergence to local optima. For these reasons, gradient-based optimizers are well-suited for problems in high-fidelity aerodynamic shape optimization, with the stipulation that gradients are evaluated in an efficient and accurate way. Examples of these optimizers include the quasi-Newton Broyden-FletcherGoldfarb-Shanno (BFGS) method [31] for unconstrained optimization, and sequential quadratic programming methods $[32,33]$ for constrained optimization.

One of the earliest applications of high-fidelity aerodynamic shape optimization was done by Hicks and Henne [34], who used finite-differences to compute objective function gradients. However, this method is computationally expensive when considering large numbers of design variables, even for the current standards in computing power. With the advent of the adjoint method (see Pironneau [35] and Jameson [36]), the cost of gradient computations has become virtually independent of the number of design variables involved.

Since then, high-fidelity aerodynamic shape optimization problems in two dimensions have been covered extensively. Anderson and Bonhaus [37] used a discrete variant of the adjoint method, i.e. the discrete-adjoint method, to compute gradients for the design of an airfoil in

2 For constrained optimization problems, gradient-based optimizers attempt to minimize the vector of gradients representing both the objective function and the constraints. 
two dimensions through the Reynolds-averaged Navier-Stokes (RANS) equations. Meanwhile, Nemec and Zingg [38] developed a gradient-based aerodynamic shape optimization algorithm that employs an efficient Newton-Krylov method for solving the RANS equations fully coupled with the one-equation Spalart-Allmaras turbulence model [39]. Driver and Zingg [40] took this a step further and implemented a laminar-turbulent transition model for the design of natural-laminar-flow airfoils.

On the other hand, high-fidelity aerodynamic shape optimization in three dimensions is still seeing rapid growth. Hicken and Zingg developed Jetstream, an Euler-based aerodynamic shape optimization algorithm in three dimensions with an integrated mesh-movement strategy [41, 42]. The capabilities of this algorithm were demonstrated through a study involving nonplanar wings exhibiting large shape changes [43]. However, despite observing substantial improvements in aerodynamic efficiency, Hicken and Zingg remarked that without the addition of viscous effects, the optimum designs were unlikely to perform well under more realistic flow conditions. Indeed, Elliott [44] postulated that aerodynamic shape optimization using the Euler equations would have the tendency to produce geometries that either exhibit boundary-layer separation or are prone to boundary-layer separation in viscous flow.

As a natural next step, Osusky and Zingg [45] adapted Jetstream for solving the RANS equations fully coupled with the one-equation Spalart-Allmaras turbulence model. In a study done by Osusky and Zingg [46], this algorithm was used to draw a comparison between RANSbased and Euler-based aerodynamic shape optimization through an application to planar wings. From this, it was found that for moderate lift coefficients, the planar wings optimized based on the RANS equations had significantly better performance in viscous flow when compared with those optimized based on the Euler equations. As anticipated by Elliott, the Euler equations were found to produce airfoils with high aft loading - a poor design for viscous flow, where more gradual pressure recoveries are preferred in order to avoid boundary-layer separation.

Following this work, Osusky et al. [47] performed RANS-based aerodynamic shape optimization on a variety of planar wings, including that of the Common Research Model (CRM), a wing-body-tail configuration with a T-tail, and a blended wing-body configuration. Koo and Zingg [48] applied the algorithm to the CRM wing-body-tail configuration, in addition to several wingletted wings sized for the Boeing 737-900; these included a winglet up configuration, a wingtip fence configuration, and a split-tip configuration. Jetstream has also been used by Reist and Zingg [18] to design and study a lifting-fuselage configuration.

More generally, three-dimensional aerodynamic shape optimization based on the RANS equations has become the new standard. For example, Jameson [49] developed the algorithm known as SYN107, which uses the continuous-adjoint method in conjunction with a finite-volume multigrid solver for solving the RANS equations. This method has been applied to the study of wing and wing-body configurations for long-range transport aircraft. SYN107 has also been used to perform aerodynamic shape optimization on transonic and supersonic business jets [50]. Meanwhile, Brezillon et al. [51] developed an unstructured flow solver and used it with the 
discrete-adjoint method to study high-lift wing configurations [52] and wing-body junctions [53]. Elsewhere, Kenway and Martins [54] used a RANS-based aerodynamic shape optimization algorithm to perform multi-point optimization studies involving the CRM wing. A similar study was done by Lyu et al. [55], in which benchmark problems were proposed.

It is clear then that the first step in evaluating the aerodynamic advantages of any unconventional aircraft configuration will be through the application of aerodynamic shape optimization based on the RANS equations. In this work, Jetstream will be used to perform RANS-based aerodynamic shape optimization on a box-wing aircraft configuration sized for regional transport. The box-wing is selected based on its merits of being aerodynamically superior to all other wing systems for a given span, height, and lift, according to linear aerodynamic theory, as well as for its compatibility with conventional fuselage and empennage designs - two characteristics that make it an attractive candidate for near-term entry into service. Given that the primary advantages of the box-wing are aerodynamic in nature, aerodynamic shape optimization based on the RANS equations will be invaluable in attempting to understand its design, as well as in assessing its ability to provide major reductions in fuel consumption, relative to the conventional cantilever-wing aircraft configuration.

\subsection{The Box Wing}

The box wing is an unconventional aircraft configuration that consists of a back-swept fore wing and a forward-swept aft wing interconnected at the wing tips by a pair of vertical fins. The fore and aft wings are of equal span and are characterized by minimal or zero dihedral, with the fore wing often positioned at the bottom of the fuselage, and the aft wing typically attached to the top of the vertical stabilizer. In this way, the box wing resembles a rectangle, or "box" when viewed from the front. As a nonplanar and closed wing system, the box wing experiences less induced drag compared to a planar wing of the same span and lift. This comes as a result of the distribution of circulation over a greater mass of air, leading to a reduction in average kinetic energy added to the flow [27], and as a result of more gradual circulation gradients, especially near the wing tips [56].

In 1924, Prandtl called the box wing the "best wing system" and described it as being superior to all other wing configurations for a given span, height, and lift [26]. This was determined through classical lifting-line theory, which was first used to demonstrate that a biplane experiences less induced drag when compared to an optimum monoplane with the same span and lift. The benefit was shown to improve when moving from a biplane to a triplane, and it was argued that this trend would continue with the further addition of lifting surfaces. In the limit of a multiplane with an infinite number of lifting surfaces, assuming the lift could be maintained, the lift distribution was found to reflect that of a box-wing configuration, namely, a closed loop circulation composed of a constant plus an elliptical distribution on the fore and aft wings, and a butterfly-shaped distribution on the vertical tip fins, as shown in Figure 1.1. 


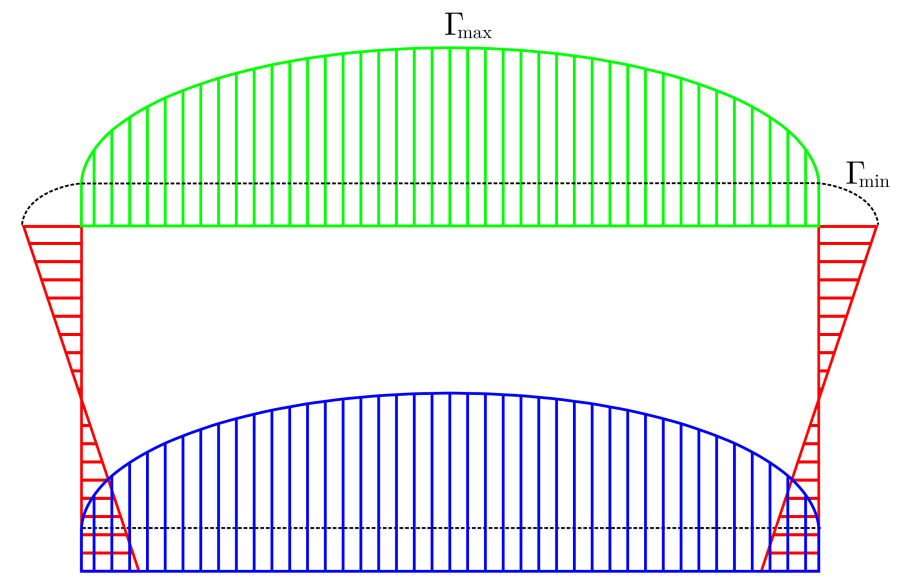

Figure 1.1: Circulation of a box wing based on linear aerodynamic theory, with the lift distribution over the fore and aft wings shown in blue and green, respectively, and the side-force distribution over the vertical tip fins shown in red.

In the same paper, Prandtl presented an equation for the induced drag experienced by a box wing, relative to that of an optimum monoplane with the same span and lift, given by

$$
\frac{1}{e} \approx \frac{1+0.45(h / b)}{1.04+2.81(h / b)}
$$

where $e$ is the span efficiency of the box wing, and $h / b$ is the height-to-span ratio. Here, the span efficiency of the optimum monoplane was taken to be unity, based on an elliptical lift distribution. Meanwhile, the nonplanar nature of the box wing allows for span efficiencies greater than one. This relationship is shown in Figure 1.2; for comparison, the relative performance of a biplane and a triplane are also shown, which illustrates that the induced drag decreases as the number of lifting surfaces tends to infinity.

Equation 1.2 indicates that for a height-to-span ratio of 0.3 , the box wing experiences only $60 \%$ of the induced drag experienced by an optimum monoplane. Since induced drag constitutes about $40 \%$ of the total drag of a commercial aircraft [27], this translates to an overall drag reduction of $16 \%$. According to Munk's stagger theorem [57], these trends apply to box wings with swept wings, as long as the lift distribution remains the same, making the box wing an attractive option for transonic aircraft.

The box-wing configuration is also robust in that it can maintain its aerodynamic efficiency at off-design conditions. Such an advantage comes from being a closed wing system, which allows a constant vortex loop to be added to the circulation with minimal penalty to induced drag [27]. Indeed, this property was corroborated by Demasi et al. [56], who found the optimum partitioning of total lift between the fore and aft wings, originally assumed to be equal by Prandtl [26], to be nonunique. Specifically, Demasi et al. demonstrated using a vortex panel method that the optimum distribution of total lift could be shifted by a constant from one wing to the other, while maintaining the same span efficiency. 


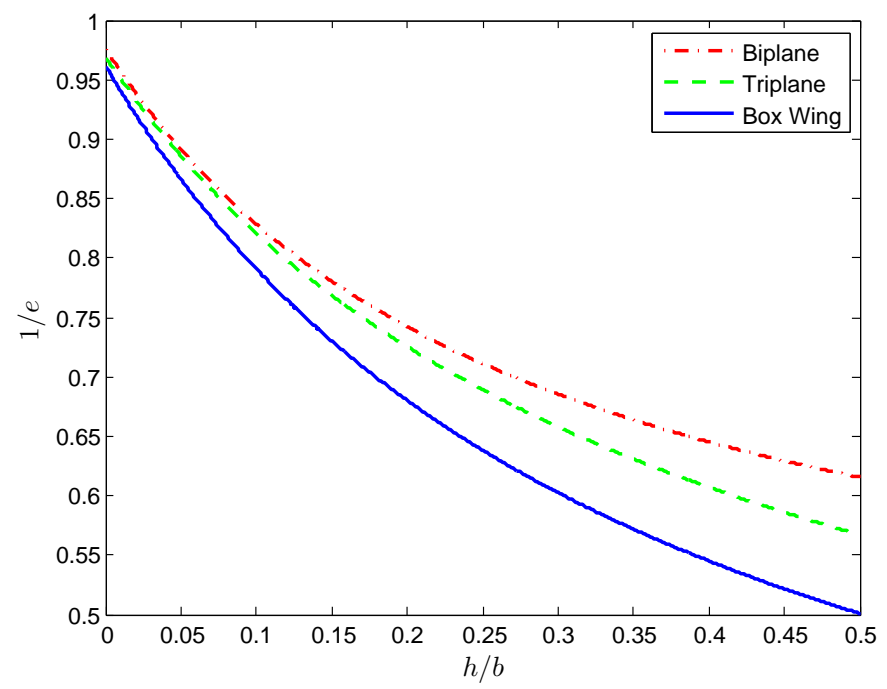

Figure 1.2: Relationship between induced drag and height-to-span ratio based on linear aerodynamic theory, relative to an optimum monoplane.

Furthermore, the box-wing configuration is attractive for reasons of safety. In particular, the box wing has exceptional longitudinal stability and control characteristics owing to its ability to provide "pure pitch," through equally effective control surfaces on the fore and aft wings [58]. The box wing also has favorable characteristics for stall recovery. This stems from the tendency of the fore wing to stall before the aft wing [59]. In such an event, the downwash on the aft wing is alleviated, allowing it to produce a large nose-down pitching moment to restore the state of the aircraft.

In 1972, Lockheed recognized the potential advantages of the box-wing configuration and initiated preliminary investigations for applications in commercial aviation. The first of these was done by Miranda [21], who recovered the aerodynamic trends predicted by Prandtl, through low speed wind tunnel tests. Subsequently, Lange et al. [60] performed a feasibility study on a long range, Mach 0.95 box-wing aircraft with a height-to-span ratio of 0.3 . A number of unique design features were considered, such as a forward-swept vertical stabilizer for accommodating a forward-shifted wing system centered about the pitch axis, and a "gull-like" aft wing, which provided a compromise between span efficiency and increased ramp weight. However, the box wing was found to experience symmetric and antisymmetric instabilities well below the target flutter speed. In addition, it was found that for a design with minimum maximum takeoff weight (MTOW), gains in aerodynamic performance were marginal, and no reduction in ramp weight could be achieved relative to a similarly-sized cantilever wing. As a result, it was concluded that the box wing might only be effective at lower Mach numbers, where structural requirements are less stringent, and flutter is less of a concern.

In 1986, Wolkovitch [22], a pioneer of the joined-wing aircraft configuration, commented on the work done by Lange et al., asserting that the poor aerodynamic performance of the box wing was likely a result of using conventional airfoil profiles in its design. In particular, 
Wolkovitch suggested that the aerodynamic advantages of the box wing, similarly with the joined wing, can only be realized if the airfoils are tailored towards the unique flow conditions experienced by the neighboring wings. Such an assessment agreed with the findings of Addoms and Spaid [59], who determined that the camber of the airfoils used in the design of biplanes must substantially differ from those used by monoplanes, as a consequence of the induced flow curvature. However, Wolkovitch conceded that the success of the box wing would ultimately hinge on a viable solution to the flutter problem.

More recently, interest in the box-wing configuration has been renewed, primarily to the credit of Frediani [58], who has been active in the development of what he calls the "PrandtlPlane." For addressing the aeroelastic instabilities, Frediani sought to increase the structural stiffness of the wing system, while ensuring that any penalty to structural weight was kept to a minimum. To this end, he proposed a tail configuration with twin vertical stabilizers, maximally distanced apart. This feature was included in a structural analysis done by Canto et al. [61], who demonstrated that a feasible box wing could be designed using aluminum structures with the same wing weight to MTOW ratio as a conventional cantilever wing. Other mitigation measures included placing more priority on structural reinforcements against out-of-plane bending moments (as also recommended by Wolkovitch [22] for the joined-wing configuration), and increasing the fore wing skin thickness [62]. Frediani went on to consider applications in commercial aviation [63, 64] and for personal use [65], with each aircraft designed for lower Mach numbers, as recommended by Lange et al. [60].

These efforts suggest that the concerns surrounding the box-wing configuration with regard to flutter and structural weight may not be as insurmountable as was initially thought. Nonetheless, further research is still required to determine whether there exists a viable solution. In the meantime, the aerodynamic performance of the box-wing configuration must also be studied in more detail. Indeed, many of the proclaimed advantages of the box wing were founded on linear aerodynamic theory; for more realistic flow conditions, however, nonlinear aerodynamics must be explored. Given that the primary advantages of the box-wing configuration are aerodynamic in nature, the evaluation of the box wing through nonlinear aerodynamics will be a key driver in deciding its future as a next generation aircraft.

Today, numerical methods in aerodynamic shape optimization have enabled in-depth studies of the box-wing configuration. One such study was done by Andrews and Perez [66], who used a low-fidelity aerodynamic shape optimization algorithm to perform a parametric study of a box-wing regional jet. Key geometric parameters were considered, such as the height-to-span ratio, the stagger-to-span ratio, and the relative planform area between the fore and aft wings. At each design point, drag minimization was performed with respect to twist and taper design variables, while subject to lift, trim, longitudinal static stability, and stall constraints. From this, several fundamental trends and trade-offs were observed. For one, it was found that the induced drag is inversely proportional to the height-to-span ratio and the stagger-to-span ratio, with both exhibiting diminishing returns at higher values; such an outcome should come as 
no surprise given that there exists a design point for which the viscous drag from the increase in wetted area eclipses the savings in induced drag. In addition, it was determined that the relative planform area had the least effect on aerodynamic performance, which agreed with the findings of Lange et al. [60]. Lastly, it was observed that the trim and longitudinal static stability constraints produced only a small penalty in total drag, as suggested by Kroo [27].

Following this work, Andrews and Perez [67] applied a low/medium-fidelity multidisciplinary optimization algorithm to the design of a box-wing aircraft based on the Bombardier CRJ-200. A cruise Mach number of 0.74 was considered at an altitude of 37,000 ft, and the optimization accounted for the complete mission profile. The framework included aerodynamics, structures, and propulsion, which allowed for interdisciplinary trade-offs. The result of the optimization was a $6 \%$ reduction in fuel burn over a similarly-sized conventional aircraft, which demonstrates the potential savings offered by regional-class box-wing jetliners.

In another recent study done by Gagnon and Zingg [68], aerodynamic shape optimization based on the Euler equations was used to evaluate the aerodynamic performance of a boxwing regional aircraft. The box wing was designed for a cruise Mach number of 0.78 and an altitude of 36,000 ft, and was based on the Bombardier CRJ-1000. For the cruise segment alone, the box wing was found to provide a $46 \%$ reduction in induced drag, relative to an equivalent optimized conventional tube-and-wing. A trade study was also presented in which a total of five optimization studies were conducted, with each subject to progressively more design variables and constraints. From this, it was found that the box wing has the freedom to redistribute the total lift from one wing to the other for satisfying design constraints while simultaneously maintaining aerodynamic performance.

Although the work of Andrews and Perez [66, 67] provided valuable insight into the fundamental trends and trade-offs of the large-scale geometric parameters of the box wing, it is believed that a number of important aerodynamic characteristics can only be uncovered through the use of high-fidelity methods. High-fidelity aerodynamic shape optimization algorithms have the particular advantage of local shape control, which allows for the detailed design of airfoils through twist, taper, and section shape degrees of freedom. This capability is of particular importance for the box wing, given that the induced flow curvature between the neighboring wings can greatly affect its performance.

Gagnon and Zingg $[68,69]$ used one such method, and suggested that the box wing is capable of providing greater improvements to aerodynamic efficiency, and therefore fuel economy, than indicated by the findings of Andrews and Perez. However, aerodynamic shape optimization at least based on the RANS equations will be necessary for capturing the intricacies of flow separation, a powerful and real phenomenon that can dictate the performance of an aircraft design $[44,47]$. The simulation of viscous effects will also allow the optimizer to account for the shorter chord lengths of the box-wing configuration, which may cause it to experience greater viscous drag compared to the cantilever wing. Finally, RANS-based aerodynamic shape optimization will enable trade-offs between parameters related to induced drag and viscous drag, 
which is of particular importance to the design of a box-wing aircraft, given the interaction of flow between the fore and aft wings, and the vertical tip fins.

\subsection{Thesis Objectives}

The aim of this work is to investigate the potential fuel burn reductions offered by the boxwing aircraft configuration relative to an equivalent conventional cantilever wing, through the application of aerodynamic shape optimization based on the RANS equations. The aerodynamic design of a box-wing and a cantilever-wing configuration will be considered, with the size and mission of each aircraft similar to the Embraer E190 regional jet.

Given that the advantages of the box-wing configuration come primarily from its wing system, the aircraft geometries will only include the wing, and the horizontal stabilizer where applicable, as done by Gagnon and Zingg [68, 69]. However, in order to better represent the relative performance of the complete aircraft, the weight and viscous drag contributions from the fuselage and the vertical stabilizer will be included post-optimization. The weight of the engines will also be accounted for, but approximations for their drag will not. These contributions are assumed to be the same for both aircraft, since in principle, the box-wing wing system is compatible with conventional fuselage and empennage designs.

As a means for ensuring the feasibility of the aerodynamic designs, a number of geometric constraints will be imposed with structural considerations in mind. These include the implementation of minimum thickness-to-chord ratio and minimum wing volume constraints, and the imposition of bounds on planform design variables such as wing sweep, height-to-span ratio, and stagger-to-span ratio.

The objective of the aerodynamic design optimizations will be to minimize drag subject to lift and trim constraints at transonic cruise conditions. For the box wing, exploratory aerodynamic shape optimization will also be performed to investigate important aerodynamic trends and trade-offs related to major design features such as wing sweep, height-to-span ratio, stagger-to-span ratio, planform area, and wetted area. Lastly, a weight sensitivity study will be performed to examine the effect that uncertainties in structural weight have on performance.

\subsection{Thesis Outline}

This thesis is organized as follows. Chapter 2 introduces the high-fidelity aerodynamic shape optimization framework known as Jetstream, with the results of its application to the design of the box-wing and cantilever-wing regional aircraft presented in Chapter 3. Chapter 4 compares the RANS-based aerodynamic shape optimization results with the Euler-based optimization results of Gagnon and Zingg $[68,69]$. Conclusions and recommendations for future work are provided in Chapter 5. 


\section{Chapter 2}

\section{Methodology}

In order to evaluate the potential fuel burn reductions offered by the box-wing aircraft configuration, a high-fidelity aerodynamic shape optimization framework known as Jetstream is used. It consists of a parameterization strategy, a geometry control system, a mesh-movement scheme, a flow solver, a gradient evaluation method, and an optimizer. Inputs include geometry definition, design mission, and weight and balance. In what follows, a brief discussion of each component will be provided. For an overview of the optimization algorithm, see Figure 2.1.

\subsection{Geometry Parameterization, Control, and Mesh Movement}

\subsubsection{B-Spline Parameterization}

In the context of exploratory shape optimization, B-spline parameterization is a flexible and efficient means for accurately representing an object of interest. A B-spline curve of degree $p$ or order $p+1$ can be expressed in parameter space as,

$$
\mathbf{C}(\xi)=\sum_{i=0}^{N} \mathcal{N}_{i}^{(p)}(\xi) \mathbf{B}_{i},
$$

where $\xi \in[0,1]$, and $\mathcal{N}_{i}^{(p)}$ are basis functions weighted by $N+1$ control points with coordinates $\mathbf{B}_{i}$. These basis functions are defined recursively through,

$$
\begin{gathered}
\mathcal{N}_{i}^{(0)}(\xi)= \begin{cases}1 & \text { if } \xi_{i} \leq \xi<\xi_{i+1} \\
0 & \text { otherwise }\end{cases} \\
\mathcal{N}_{i}^{(p)}(\xi)=\frac{\xi-\xi_{i}}{\xi_{i+p}-\xi_{i}} \mathcal{N}_{i}^{(p-1)}(\xi)+\frac{\xi_{i+p+1}-\xi}{\xi_{i+p+1}-\xi_{i+1}} \mathcal{N}_{i+1}^{(p-1)}(\xi),
\end{gathered}
$$




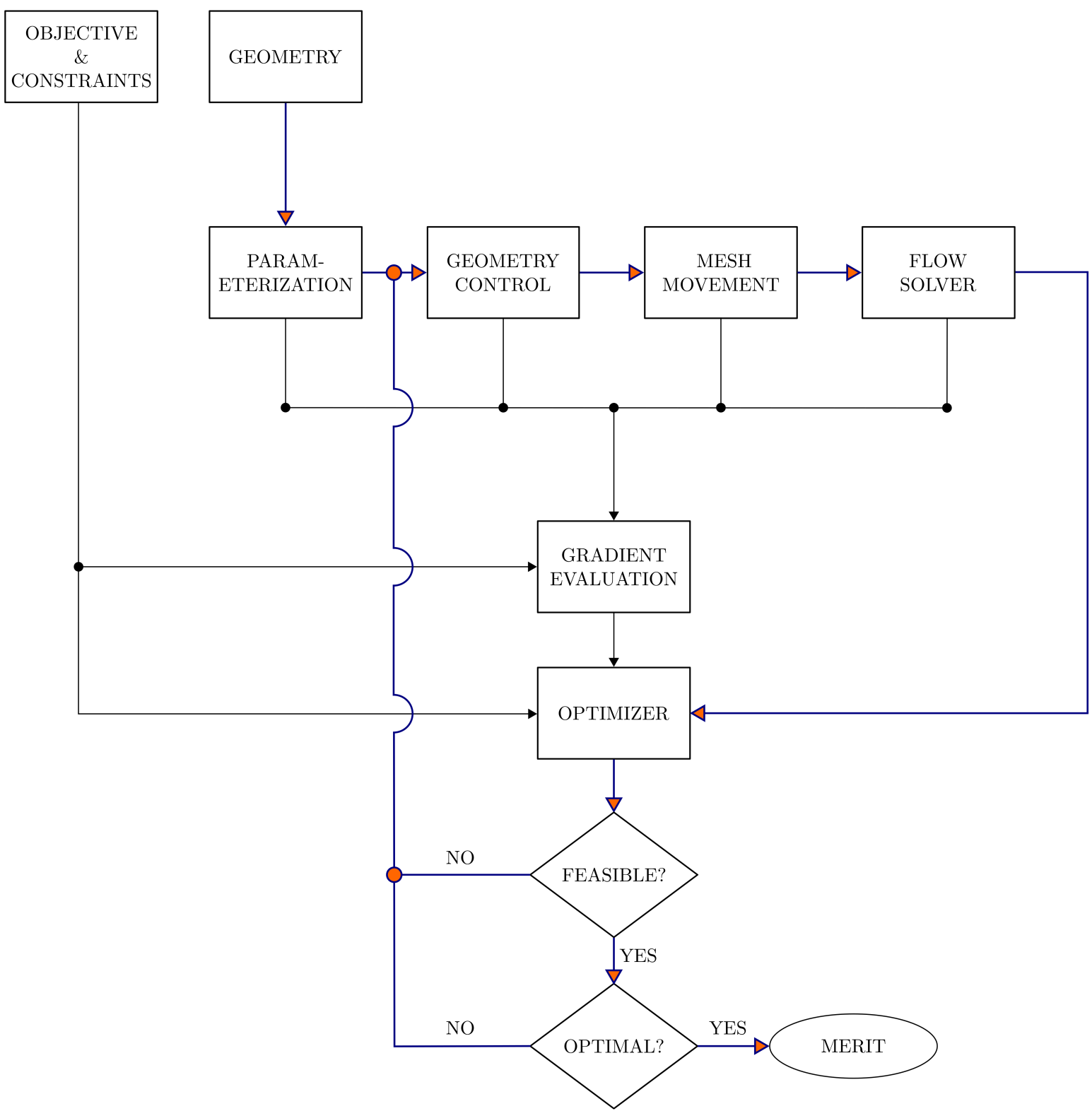

Figure 2.1: A flowchart of Jetstream.

and a knot vector of size $N+p+1$ given by,

$$
T=\{\underbrace{0, \ldots, 0}_{p+1}, \xi_{p+1}, \ldots, \xi_{N}, \underbrace{1, \ldots, 1}_{p+1}\}
$$

that specifies the locations of the knots at which the basis functions are joined. At each knot, the basis functions are $C^{p-k}$ continuous, where $k$ is the multiplicity of a given knot, while between each knot, the basis functions are infinitely differentiable [70]. The $p+1$ repeated knots at the ends of the vector (i.e. $k=p$ ) ensure that the curve intersects with the end control points $\mathbf{B}_{0}$ and $\mathbf{B}_{N}$. This guarantees at least $C^{0}$ continuity where individual B-spline curves 
are connected and can readily be shown through Equation 2.2 and Equation 2.3, which yields $\mathcal{N}_{0}^{(p)}(0)=\mathcal{N}_{N+p+1}^{(p)}(1)=1$. This property allows for the use of piecewise B-spline curves to represent more complicated shapes, while remaining watertight.

The definition of a B-spline curve, in addition to all of its properties, can be extended to represent objects of higher dimension such as surfaces and volumes, through tensor products. For instance, a B-spline volume is defined as,

$$
\mathbf{V}(\boldsymbol{\xi})=\sum_{i=0}^{N_{\xi}} \sum_{j=0}^{N_{\eta}} \sum_{k=0}^{N_{\zeta}} \mathcal{N}_{i}^{(p)}(\xi) \mathcal{N}_{j}^{(q)}(\eta) \mathcal{N}_{k}^{(r)}(\zeta) \mathbf{B}_{i, j, k},
$$

where $\boldsymbol{\xi}=(\xi, \eta, \zeta) \in[0,1]^{3}$ are the parametric coordinates.

B-spline curves, surfaces, and volumes play an important role in Jetstream. They are involved in the parameterization of the aerodynamic surfaces, the geometry control system, and the mesh movement algorithm, and their properties contribute to the overall success of the optimization suite. This will become clear throughout the remainder of the chapter.

\subsubsection{Grid Fitting and Grid Refinement}

The parameterization of the computational domain involves a grid fitting procedure in which each block of the multi-block mesh is parameterized with a B-spline volume. These B-spline volumes are cubic in $\xi, \eta$ and $\zeta$, and follow a chord-length-based parameterization. In defining the knot vector, an equal number of grid nodes are made to be contained within each knot interval. This results in a B-spline volume control grid that adopts the spatial distribution of the grid nodes - an important property for the integrated mesh-movement scheme discussed later in Section 2.1.4. The coordinates of the control points are determined from a least squares fit, with block edges parameterized first, followed by surfaces, and then volumes. This ordering ensures that the location of each control point is consistent between adjacent block interfaces.

For flow analyses based on the RANS equations, an accurate representation of the boundary layer requires fine off-wall spacings. This poses a challenge during the grid fitting process since the fitting error is often greater than the off-wall spacing by two or more orders of magnitude. This can lead to issues such as control point cross-over, which gives rise to negative volumes within the computational domain. To overcome this, the B-spline volume control grid is first fit to a mesh with coarsened off-wall spacings. The control points are then frozen while the parameterization is redefined by the grid node distribution of a grid suitable for computations of turbulent flows. The result is an algebraic approximation of the grid for turbulent flow that is not susceptible to control point cross-over [47].

The parameterization of the computational domain also allows for grid refinement. This is accomplished through a combination of node insertion and node redistribution, hence increasing grid resolution while maintaining the spacing function of interest. In this work, grid refinement is used to perform Richardson extrapolation with an assumed second order for obtaining grid 
converged aerodynamic functionals. This is done following an optimization on a coarser grid, which would otherwise be computationally expensive. To ensure that the aerodynamic trends remain intact, the optimization grids are sufficiently well-refined such that the ratio between pressure and friction drag is close to the grid converged value.

\subsubsection{Free-Form and Axial Deformation Geometry Control}

Free-form deformation (FFD) is a geometry control method that is attractive for its ability to provide rapid and smooth deformations, and for its mathematical intuitiveness. The method employs FFD volumes, defined as B-spline volume lattices, that transform an embedded object of interest as the FFD-volumes themselves are deformed. In this way, the shape deformation process is dissociated from the shape representation of the embedded object, which allows for the use of fewer yet more intuitive design variables. As such, design problems involving FFD geometry control are often less encumbered by geometric constraints, making for more flexible design spaces that are well-suited for exploratory shape optimization.

FFD can be described mathematically as a mapping from physical space, $\boldsymbol{t}$, to parameter space, $\boldsymbol{\xi}$, to deformed physical space, $\tilde{\boldsymbol{t}}$. In practice, this is implemented through two functions. The first function maps the vertices, $\boldsymbol{t}$, of the embedded object to parameter space, $\boldsymbol{\xi}$, through a Newton search procedure, and is only performed once. The second function transforms the coordinates of the vertices in parameter space, $\boldsymbol{\xi}$, to deformed physical space, $\tilde{\boldsymbol{t}}$, following a perturbation of the FFD-volume lattice. This process can be expressed as the solution to,

$$
\mathcal{F}(\boldsymbol{t}, \tilde{\boldsymbol{t}})=\tilde{F}\left(F^{-1}(\boldsymbol{t})\right)-\tilde{\boldsymbol{t}}=\tilde{F}(\boldsymbol{\xi})-\tilde{\boldsymbol{t}}=0,
$$

where the first and second functions are given by $F^{-1}$ and $\tilde{F}$, respectively.

As with Gagnon and Zingg [71], the surface control points are embedded within the FFD volumes rather than the surface discretization, as is often done. This ensures that the analytical representation of the underlying surface is retained; an important feature for gradient-based optimizers such as the one used in this work, which necessitates a smooth design space. By embedding the surface control points, the aerodynamic shape can also be regenerated quickly, following an update to the geometry control system.

For application to wing geometries, the FFD geometry control system used by Jetstream allows for changes to twist, taper, and section shape. This is done through rotation and scaling operators that are $4 \times 4$ matrices given by $\mathbf{R}(\tilde{o}, \theta, \boldsymbol{u})$ and $\mathbf{S}(\tilde{o}, c, \boldsymbol{u})$, respectively. Here, $\tilde{o}$ is the local origin which defines the rotation or scaling, $\theta$ is the rotation angle, $c$ is the scaling factor, and $\boldsymbol{u}$ is an optional unit vector that specifies the axis of rotation or the direction of scaling. In particular, for a given FFD-volume cross-section, the degrees of freedom are defined as follows.

- twist: a rotation of the FFD-volume cross-section in the local $x z$-plane about the local origin $\tilde{o}, \mathbf{R}(\tilde{o}, \theta,[0,1,0])$. The design variable is $\theta$. 
- taper: a uniform scaling in the local $x z$-plane from the local origin $\tilde{o}$ along the local $x$ and $z$-axes, $\mathbf{S}\left(\tilde{o}, c_{x z},[1,0,1]\right)$ and $\mathbf{S}\left(\tilde{o}, c_{x z},[1,0,-1]\right)$. The design variable is $c_{x z}$.

- section shape: a nonuniform scaling in the local $x z$-plane along the local $z$-axis and away from the line-of-symmetry for a given control point, $i, \mathbf{S}\left(\tilde{o}, c_{z_{i}},[0,0, \pm 1]\right)$. The design variables are $c_{z_{i}}$.

These transformation operators are applied to the initial $x y z$-coordinates of the FFD-volume lattice at each update, with the changes linearly interpolated between each FFD-volume crosssection. By defining the taper degree of freedom as a uniform scaling, the thickness-to-chord ratio determined by the section shape design variables is maintained as taper is varied. To be clear, the design variables of each FFD-volume cross-section are independent from one another, unless otherwise constrained geometrically.

The FFD design variables are illustrated in Figure 2.2 for a given FFD-volume cross-section. Here, a wing geometry is parameterized with B-spline surfaces, and the surface control points are embedded within an FFD volume. As changes are made to the coordinates of the FFD control points, the FFD volumes deform the underlying aerodynamic surfaces.

\section{Axial Deformation}

Axial deformation can be thought of as an extension of FFD that makes use of an axial curve, defined as a B-spline, to provide additional degrees of freedom. Originally conceived by Lazarus et al. for applications in computer graphics [72], the method has since been applied to aerodynamic shape optimization through the work of Gagnon and Zingg [68, 69]. Unlike the original formulation, the vertices of the embedded object are mapped to the FFD-volume lattices as described above, as opposed to the axial curve. Then, the FFD-volume lattices are mapped to the nearest point on the axial curve. Hence, the FFD volumes and the axial curve are decoupled insofar as their mathematical definitions are concerned.

The FFD-volume lattices can be thought of as being attached to the axial curve, which is usually positioned at either the leading edge, the trailing edge, or the quarter-chord of the wing. In such a way, one or more axial curves can provide sweep, span, and dihedral degrees of freedom. This is implemented through a translation operator, $\mathbf{T}(\boldsymbol{w})$, where $\boldsymbol{w}$ is a vector describing the changes in the $x y z$-coordinates of an axial curve control point. Formally, the degrees of freedom for a given axial curve control point are defined as follows.

- sweep: a change in the $x$-coordinate of an axial curve control point, $\mathbf{T}([\Delta x, 0,0])$. The design variable is $\Delta x$.

- span: a change in the $y$-coordinate of an axial curve control point, $\mathbf{T}([0, \Delta y, 0])$. The design variable is $\Delta y$.

- dihedral: a change in the $z$-coordinate of an axial curve control point, $\mathbf{T}([0,0, \Delta z])$. The design variable is $\Delta z$. 


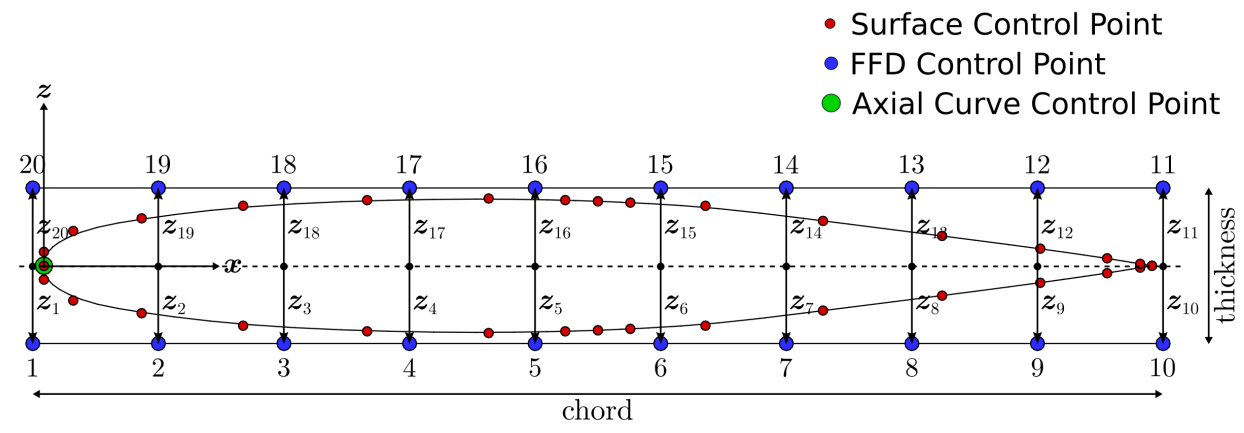

(a) Initial

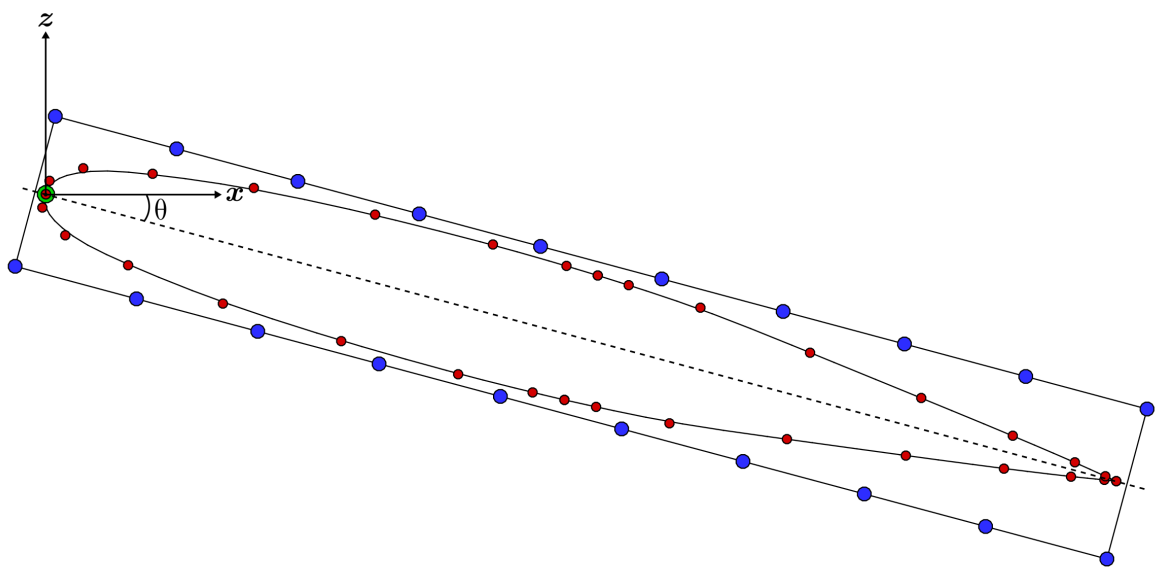

(b) Twist

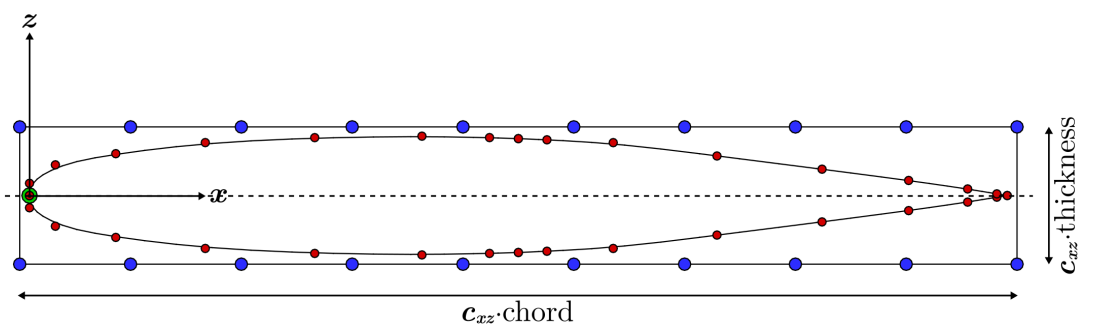

(c) Taper

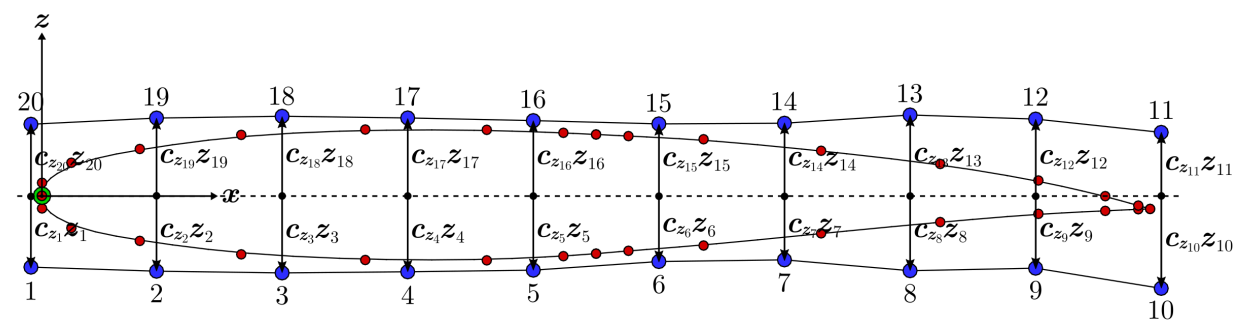

(d) Section shape

Figure 2.2: The FFD geometry control system design variables for an airfoil. 


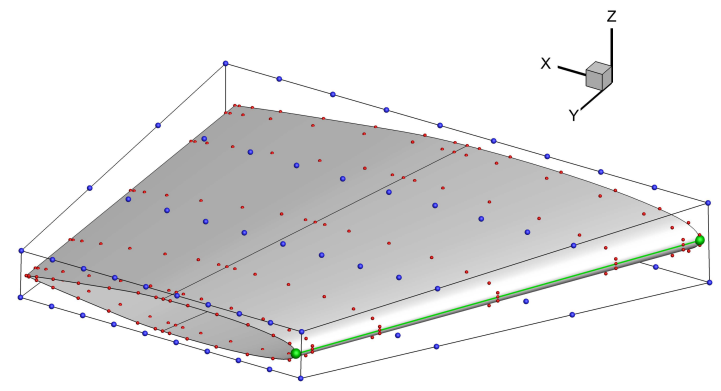

(a) Initial

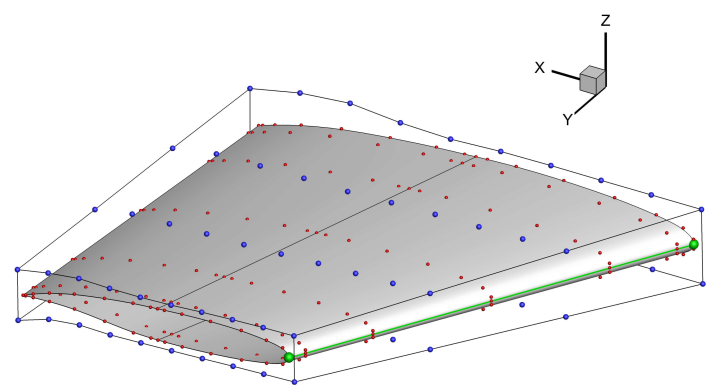

(b) Deformed

Figure 2.3: A free-form and axial deformation geometry control system setup for a wing section which includes surface control points (red spheres), FFD volumes (black lines), FFD control points (blue spheres), axial curves (green lines), and axial curve control points (green spheres).

To obtain the perturbed $x y z$-coordinates of the axial curve control points, the design variables are added to the corresponding initial $x y z$-coordinates of the axial curve control points at each update. As the axial curves are deformed, the local coordinate systems of the FFDvolume cross-sections translate with the axial curves, as one would expect. Furthermore, the FFD-volume cross-sections are reoriented such that they remain perpendicular to their respective axial curve. Although the local origins need not coincide with the positions of the axial curves, they are chosen to do so in this work for convenience. Similar to the FFD design variables, the design variables of each axial control point are independent, unless subject to a geometric constraint.

Figure 2.3a illustrates a three-dimensional example of how the geometry control system can be set up for a wing. A section of a parameterized wing is embedded within an FFD volume with four cross-sections. Each cross-section is attached to a linear axial curve positioned at the leading edge of the wing. As shown in Figure 2.3b, the embedded wing section undergoes smooth deformation as the FFD control points are perturbed.

As a final note, axial curves that consist of more than two control points can open the possibility for nonlinear planform designs. This can provide the optimizer with additional mechanisms for achieving an optimal spanwise lift distribution. Interesting as that may be, however, this work will consider only linear axial curves (when possible) and rely on the FFD design variables for deriving optimally-loaded aerodynamic surfaces.

\subsubsection{Mesh Movement Scheme}

Once the aerodynamic surfaces have been deformed, a means must be provided for updating the computational domain. In Jetstream, this is accomplished through a robust and efficient integrated mesh-movement scheme based on the theory of linear elasticity. For this strategy, each block of the computational domain is parameterized with a B-spline volume, as described in Section 2.1.2. This results in a grid of volume control points, $\mathbf{b}$, which mimics the spatial 
distribution of the grid nodes. The mesh-movement scheme can then be applied to the coarse grid of volume control points rather than the grid nodes themselves, without loss of generality. This leads to a significant reduction in computational cost, since the number of volume control points is typically fewer than the number of grid nodes by two to three orders of magnitude. To tightly integrate mesh movement and surface deformation, the subset of volume control points that lie on the boundaries of the aerodynamic surfaces are positioned to coincide with the surface control points, $\mathbf{A} \subset \mathrm{b}$. This is illustrated in Figure 2.4a where the surface control points of a cantilever wing intersect with the nodes of the coarse control net. The corresponding fine grid is shown in Figure 2.4b.

The reduction in computational cost offered by the coarse control net is leveraged to implement a robust mesh-movement scheme based on the theory of linear elasticity. This method models each B-spline volume as a linear elastic solid and is given by

$$
\mathcal{M}^{(i)}=\mathbf{K}^{(i)}\left(\mathbf{b}^{(i)}-\mathbf{b}^{(i-1)}\right)-\mathbf{f}^{(i)}=0, \quad i=1,2, \ldots, m
$$

where $\mathcal{M}$ is the mesh residual, $\mathbf{K}$ is the stiffness matrix, $\mathbf{b}$ is a block-column vector that represents the coordinates of the volume control points, and $\mathbf{f}$ is the force vector which implicitly depends on the changes in the surface control point coordinates, i.e. $\Delta \mathbf{A}^{(i)}=\mathbf{A}^{(i)}-\mathbf{A}^{(i-1)}$. A large shape change can be achieved by subdividing the mesh movement into a total of $m$ linear increments through,

$$
\mathbf{A}^{(i)}=\frac{i}{m}\left(\mathbf{A}^{(m)}-\mathbf{A}^{(0)}\right)+\mathbf{A}^{(0)}, \quad i=1,2, \ldots, m,
$$

thus satisfying the small strain assumption.

For the stiffness matrix, element stiffness is defined by Young's modulus, which is tailored to maintain the quality of cells bordering the aerodynamic surfaces, i.e. the boundary layer. It is given by

$$
E_{\varepsilon}^{(i)}=\frac{\Phi_{\varepsilon}^{(i-1)}}{\Phi_{\varepsilon}^{(0)} V_{\varepsilon}^{(i-1)}}, \quad i=1,2, \ldots, m,
$$

where $V_{\varepsilon}$ is a volume element and $\Phi_{\varepsilon}$ is an orthogonality measure [42] given by

$$
\Phi_{\varepsilon}=\left(\prod_{v=1}^{8} \frac{1}{\left(\boldsymbol{u}_{v} \times \boldsymbol{v}_{v}\right) \cdot \boldsymbol{w}_{v}}\right)^{2},
$$

where the vectors $\boldsymbol{u}_{v}, \boldsymbol{v}_{v}$, and $\boldsymbol{w}_{v}$ are unit vectors parallel to the element edges that meet at vertex $v$. Note that $\left(\boldsymbol{u}_{v} \times \boldsymbol{v}_{v}\right) \cdot \boldsymbol{w}_{v}$ is unity when the edges are orthogonal and zero when the edges are coplanar. In addition, Equation 2.8 ensures that small volume elements are stiffer than large volume elements.

Equation 2.6 is solved using the conjugate-gradient method. This method is preconditioned using incomplete lower-upper factorization with a fill level of 1, i.e. ILU(1) [73]. For more details 


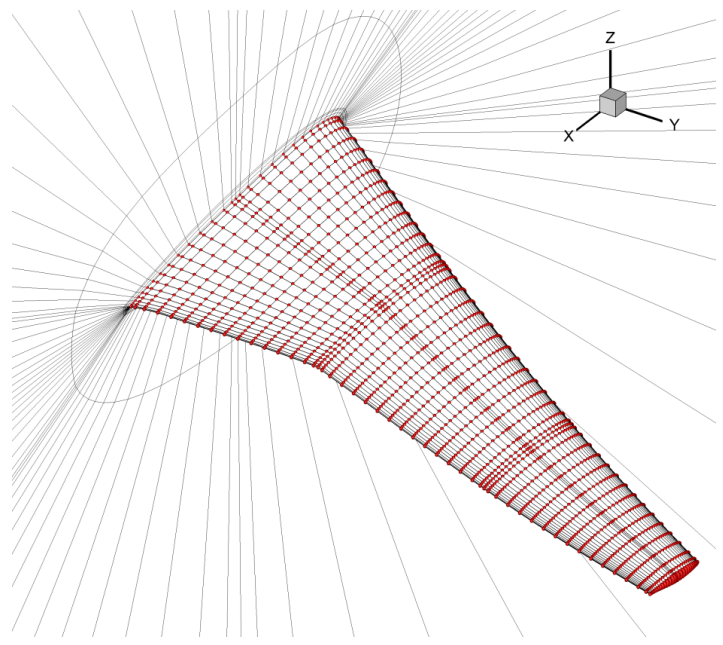

(a) Control grid

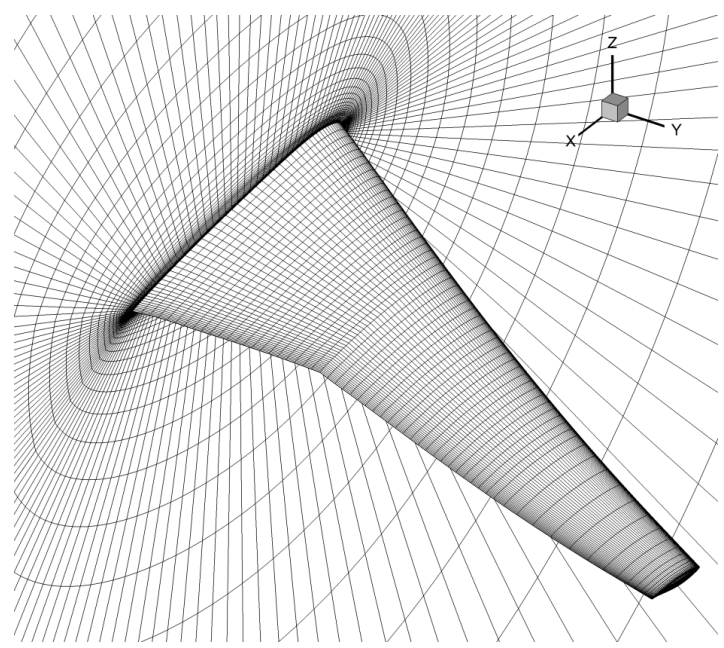

(b) Fine grid

Figure 2.4: The control grid overlayed with the surface control points (red spheres) [left], and the corresponding fine grid [right] for a cantilever wing.

on the integrated mesh movement algorithm, see Hicken and Zingg [42].

\subsection{Flow Solver}

In order to determine the aerodynamic performance of a given aircraft, a three-dimensional structured multi-block flow solver is used to compute the aerodynamic functionals. This algorithm is an implicit parallel Newton-Krylov-Schur flow solver that is capable of solving either the Euler or the Reynolds-averaged Navier-Stokes (RANS) equations. In this work, the RANS equations are considered, and closure is provided by a full coupling of the one-equation SpallartAllmaras turbulence model [39]. Although a laminar-turbulent transition point can be specified, boundary layers are assumed to be fully turbulent.

The RANS equations are discretized through second-order centered difference summationby-parts (SBP) operators [74]. These are used in conjunction with simultaneous-approximation terms (SATs) which enforce boundary conditions and inter-block coupling weakly. Together, SBP-SAT operators provide excellent numerical stability properties and efficient parallel performance, while only requiring $C^{0}$ mesh continuity at block interfaces [75]; recall from Section 2.1.1 that this condition is met by the definition of the knot vector. To aid in stabilizing the solution around shocks, a pressure sensor is used to control the activation of artificial dissipation. This work uses 2nd- and 4th-difference scalar dissipation operators [76, 77], but matrix dissipation is also available [78].

Once the flow equations have been transformed to curvilinear coordinates and discretized in space, they can be expressed as the ordinary differential equation,

$$
\frac{d \mathbf{q}}{d t}=\mathcal{R}\left(\mathbf{q}, \mathbf{b}^{(m)}\right)
$$


where $\mathbf{q}$ is the block-column vector of mean conservative flow variables and the turbulence variable, and $\mathcal{R}$ is the vector of flow residuals that consists of the inviscid and viscous flux terms, the artificial dissipation, and the turbulence model. To this, a local time linearization is applied, which gives

$$
\left(\mathcal{A}^{(n)}+\frac{\mathbf{I}}{\Delta t^{(n)}}\right) \Delta \mathbf{q}^{(n)}=\mathcal{R}^{(n)},
$$

where $\mathcal{A}=\partial \mathcal{R} / \partial \mathbf{q}$ is the flow Jacobian, $\mathbf{I}$ is the identity matrix, $\Delta t$ is the time step, and $n$ is the time-step index. The steady-state solution is then computed using Newton's method.

Newton's method is an iterative method that offers quadratic convergence so long as a sufficiently close initial iterate is provided. In order to arrive at a suitable initial guess, Newton's method is divided into two phases: an approximate-Newton phase and an inexact-Newton phase. In the first phase, an approximate Jacobian is constructed and is used to solve the nonlinear set of equations until the mean flow residual has been reduced by several orders of magnitude, i.e. to a relative tolerance of $10^{-4}$ or $10^{-5}$. In the second phase, an inexact Jacobian is constructed and is used to drive the initial iterate obtained from the approximate-Newton phase to steady-state, namely, to a relative tolerance of $10^{-8}$ or an absolute tolerance of $10^{-6}$. The linear system that arises from each Newton phase is solved using a flexible variant of the Krylov subspace method, generalized minimal residual (GMRES) [79, 80], and is subject to Schur preconditioning [81].

To speed up convergence, local time-stepping is used. In addition, pseudo-transient continuation is applied to provide time-step acceleration. For more details on the flow solver, see Hicken and Zingg [41] and Osusky and Zingg [45].

\subsection{Gradient Evaluation and Optimization Algorithm}

\subsubsection{The Optimization Problem}

In general, aerodynamic shape optimization problems are formulated as minimization problems with respect to a set of design variables subject to a number of constraints. In this work, the problem statement can be written as

$$
\begin{aligned}
\operatorname{minimize} & \mathcal{J}\left(\mathbf{v}, \mathbf{q}, \mathbf{b}^{(m)}\right) \\
\text { w.r.t. } & \mathbf{v}, \\
\text { s.t. } \quad & \mathcal{M}^{(i)}\left(\mathbf{A}^{(i)}(\mathbf{v}), \mathbf{b}^{(i)}, \mathbf{b}^{(i-1)}\right)=0, \quad i=1,2, \ldots, m, \\
& \mathcal{R}\left(\mathbf{v}, \mathbf{q}, \mathbf{b}^{(m)}\right)=0, \\
& \mathcal{C}_{\mathrm{e}}\left(\mathbf{v}, \mathbf{q}, \mathbf{b}^{(m)}\right)=0, \quad \mathcal{C}_{\mathrm{i}}\left(\mathbf{v}, \mathbf{q}, \mathbf{b}^{(m)}\right) \geq 0
\end{aligned}
$$

where $\mathcal{J}$ is the objective function, $\mathbf{v}=\left[\alpha, \mathrm{x}_{\mathrm{CG}}, \mathrm{z}_{\mathrm{CG}}, \mathbf{v}_{\text {geo }}^{\mathrm{T}}\right]^{\mathrm{T}}$ is the vector of design variables, $\left\{\mathcal{M}^{(i)}\right\}_{i=1}^{m}$ are the mesh movement residuals, $\mathcal{R}$ is the flow residual, and $\mathcal{C}_{\mathrm{e}}$ and $\mathcal{C}_{\mathrm{i}}$ are addi- 
tional equality and inequality constraints, respectively, which can be either linear or nonlinear. Examples of $\mathcal{C}_{\mathrm{e}}$ and $\mathcal{C}_{\mathrm{i}}$ include constant lift, trimmed flight, and minimum wing volume. Other geometric constraints can also be considered, such as those that are imposed on the FFD and axial curve control points, and the center of gravity (CG).

For high-fidelity aerodynamic shape optimization problems in three dimensions, design spaces are often complicated, given the large number of design variables involved. Indeed, design spaces for these problems are likely multimodal [82]. Global optimization strategies such as genetic algorithms [29] and surrogate-assisted methods [30] are attractive for this reason, but are computationally expensive when considering hundreds of design variables. As an alternative, gradient-based algorithms offer fast convergence while not being cost prohibitive. These methods are deterministic, and follow a path based on the gradient of the objective function for unconstrained optimization problems, and the gradient of both the objective function and the constraints for constrained optimization problems.

A challenge associated with gradient-based methods is that they cannot guarantee the discovery of the global optimum. This can be addressed by applying a multi-start method, which begins the optimization problem from a number of distinct initial conditions [82]. Nevertheless, only single-start optimization is considered in this work, which may be sufficient for understanding trade-offs between competing parameters.

To increase the probability of finding the global optimum, care is taken when designing the initial geometry. Geometric constraints on the design variables are also selected with the intent of guiding the design towards the best possible optimum. With that said, one must be cognizant that the optimization problem can only uncover novel aircraft designs if the degrees of freedom are not excessively inhibited.

\subsubsection{Gradient Evaluation}

Three-dimensional aerodynamic shape optimization problems often involve large numbers of design variables. This can lead to proportionately high computational costs when evaluating gradients through finite-difference methods. As an alternative, the discrete-adjoint method [35, 36,37 is capable of computing these gradients at a cost that is virtually independent of the number of design variables.

The method is formulated through the Lagrangian of the objective function, $\mathcal{J}$, and is given by

$$
\mathcal{L}=\mathcal{J}\left(\mathbf{v}, \mathbf{b}^{(m)}, \mathbf{q}\right)+\sum_{i=1}^{m} \boldsymbol{\lambda}^{(i) \mathrm{T}} \mathcal{M}^{(i)}\left(\mathbf{A}^{(i)}(\mathbf{v}), \mathbf{b}^{(i)}, \mathbf{b}^{(i-1)}\right)+\boldsymbol{\psi}^{\mathrm{T}} \mathcal{R}\left(\mathbf{v}, \mathbf{b}^{(m)}, \mathbf{q}\right),
$$

where the mesh movement and flow equations are imposed as constraints with Lagrange multipliers or adjoint variables, $\left\{\boldsymbol{\lambda}^{(i)}\right\}_{i=1}^{m}$ and $\boldsymbol{\psi}$, respectively. The partial derivatives $\partial \mathcal{L} / \partial \boldsymbol{\lambda}^{(i)}$ and $\partial \mathcal{L} / \partial \boldsymbol{\psi}$ recover the mesh movement and flow equations, which tend to zero as they are converged. These two conditions are a subset of the first-order Karush-Kuhn-Tucker (KKT) conditions that must be satisfied for optimality [31]. 
Another two conditions are obtained by setting to zero the partial derivatives of $\mathcal{L}$ with respect to $\mathbf{b}^{(m)}$ and with respect to $\mathbf{q}$. This yields

$$
\begin{aligned}
\left(\frac{\partial \mathcal{R}}{\partial \mathbf{q}}\right)^{\mathrm{T}} \boldsymbol{\psi} & =-\left(\frac{\partial \mathcal{J}}{\partial \mathbf{q}}\right)^{\mathrm{T}} \\
\left(\frac{\partial \mathcal{M}^{(m)}}{\partial \mathbf{b}^{(m)}}\right)^{\mathrm{T}} \boldsymbol{\lambda}^{(m)} & =-\left(\frac{\partial \mathcal{J}}{\partial \mathbf{b}^{(m)}}\right)^{\mathrm{T}}-\left(\frac{\partial \mathcal{R}}{\partial \mathbf{b}^{(m)}}\right)^{\mathrm{T}} \boldsymbol{\psi} \\
\left(\frac{\partial \mathcal{M}^{(i)}}{\partial \mathbf{b}^{(i)}}\right)^{\mathrm{T}} \boldsymbol{\lambda}^{(i)} & =-\left(\frac{\partial \mathcal{M}^{(i+1)}}{\partial \mathbf{b}^{(i)}}\right)^{\mathrm{T}} \boldsymbol{\lambda}^{(i+1)}, \quad i=m-1, m-2, \ldots, 1
\end{aligned}
$$

Equation 2.13 is the flow-adjoint equation, and Equations 2.14 and 2.15 are the mesh-adjoint equations. For the flow-adjoint equation, the flow Jacobian matrix is linearized by analytically differentiating the viscous fluxes, the artificial dissipation, and the turbulence model while the complex-step method $[83,84]$ is used for the boundary conditions. The flow-adjoint system is solved using a modified, flexible version of GCROT [85, 86], while the mesh-adjoint system is solved using the conjugate-gradient method preconditioned with ILU(1).

The adjoint equations are solved sequentially in the order presented. This enables the computation of the gradient, which is given by the partial derivative of $\mathcal{L}$ with respect to the design variables, $\mathbf{v}$ :

$$
\mathcal{G} \equiv \frac{\partial \mathcal{L}}{\partial \mathbf{v}}=\frac{\partial \mathcal{J}}{\partial \mathbf{v}}+\sum_{i=1}^{m}\left(\boldsymbol{\lambda}^{(i) \mathrm{T}} \frac{\partial \mathcal{M}^{(i)}}{\partial \mathbf{A}^{(i)}} \frac{\partial \mathbf{A}^{(i)}}{\partial \mathbf{A}^{(m)}} \frac{\partial \mathbf{A}^{(m)}}{\partial \mathbf{v}}\right)+\boldsymbol{\psi}^{\mathrm{T}} \frac{\partial \mathcal{R}}{\partial \mathbf{v}}
$$

where $\mathcal{G}$ denotes the gradient of the objective function, and the surface sensitivites are given by

$$
\frac{\partial \mathbf{A}^{(m)}}{\partial \mathbf{v}}=\frac{\partial \mathbf{A}^{(m)}}{\partial \mathbf{B}} \frac{\partial \mathbf{B}}{\partial \mathbf{v}}
$$

where $\mathbf{B}$ are the FFD-volume control point coordinates. Here, $\partial \mathbf{A}^{(m)} / \partial \mathbf{B}$ is calculated exactly from the B-spline volume definitions (Equation 2.4), and $\partial \mathbf{B} / \partial \mathbf{v}$ is calcuated using the complexstep method [71]. The system represented by Equation 2.16 is nonlinear and is driven to convergence by the gradient-based optimization algorithm.

For each additional nonlinear constraint that depends on the flow equations, the gradients must also be evaluated through Equation 2.16. In this work, these include the lift and trim constraints. For constraints that are independent of the flow equations, the gradients are calculated analytically.

\subsubsection{SNOPT}

Jetstream is interfaced with a software package known as SNOPT [32], which is a gradientbased optimization algorithm that uses sequential quadratic-programming. It is capable of 
solving nonlinear problems subject to both linear and nonlinear constraints, with the former satisfied exactly at the start of the optimization. SNOPT provides the option of approximating gradients through finite-difference schemes, but in this work, gradients are calculated in advance and passed to SNOPT. This improves accuracy and reduces computational cost, given that the gradients are computed either analytically or by using the discrete-adjoint method. SNOPT approximates the Hessian of its own Lagrangian using the BFGS quasi-Newton method.

SNOPT provides a number of metrics for tracking convergence. Of particular interest are Feasibility, Merit, and Optimality. Feasibility represents how well the nonlinear constraints are satisfied, while Merit represents a combination of Feasibility and the objective function, and corresponds to the latter when Feasibility is met. Optimality represents the gradient of the augmented objective function, which is driven towards zero at a local or global optimum. In this work, aerodynamic designs are considered optimal when the Merit function aymptotes, Feasibility has been satisfied to an absolute tolerance of $10^{-5}$ or $10^{-6}$, and Optimality has reduced by at least two orders of magnitude. Deep convergence of Optimality is preferred, but is often difficult to achieve for constrained optimization problems in three dimensions.

\subsection{Geometry Definition}

The aerodynamic surfaces of each aircraft are created using a geometry-definition tool known as Genair [87]. Genair takes a component-based approach in which each object is modeled with watertight generalized B-splines. Genair is also used to set up the free-form and axial deformation geometry control system; this includes the embedding of surface control points within FFD volumes and the mapping of the FFD volumes to the axial curves. Note that the former can only be performed once the surface meshes have been parameterized with B-splines, as described in Section 2.1.2.

\subsection{Design Mission}

The size and design mission of each aircraft is based on the Embraer E190 regional jet. Steady level flight is considered at a Mach number of 0.78 and an altitude of 36,000 ft. A nominal cruise mission is considered where each aircraft is to transport 100 passengers and 5 crew over

a distance of $500 \mathrm{nmi}$, with a $100 \mathrm{nmi}$ fuel reserve as per FAR part 21 . The weight of each aircraft is taken to be the maximum weight at cruise.

Performance is evaluated using the Breguet range equation (cruise/climb) given by Equation 1.1. Of particular interest is the reduction in fuel burn offered by the box-wing configuration, relative to the cantilever-wing aircraft. This is represented by the difference in fuel burn between the two aircraft divided by the fuel consumed by the conventional baseline. Each aircraft is driven by a pair of CF34-10E engines that each have a thrust specific fuel consumption of $0.64 \mathrm{lb} / \mathrm{lbf} / \mathrm{h}$. 


\subsection{Weight and Balance}

The weight and CG of an aircraft are two important parameters that define steady level flight. In particular, the weight of an aircraft determines the lift needed to maintain flight at a given altitude, while the CG of an aircraft is the point about which the aircraft must be balanced for trimmed flight. Because of this, the method chosen to approximate these parameters can greatly affect the predicted performance of the aircraft. For example, if the approximation leads to an overestimate of the aircraft weight, the performance of the aircraft will suffer a penalty in fuel consumption, both directly through the requirement of a greater lift, and indirectly from the resulting increase in induced drag. On the other hand, if the aircraft weight is underestimated, it can become unclear whether the gains in performance came from the advantages of the design, or from the limitations of the approximation. Similarly, if the CG of an aircraft is not accurately captured, the performance can be affected either positively or negatively, depending on the trimming moment required by the horizontal stabilizer to keep the aircraft level.

In this work, the majority of the weight and balance calculations are done using methods based on the work of Torenbeek [88], Raymer [89], and Roskam [90]. These relationships are empirical formulae based on conventional aircraft data that model the weight and CG of the major aircraft components and account for the aircraft systems, structures, payload, fuel and operational items. Though of low fidelity, these approximations are well-suited for the preliminary design stage, during which detailed information is often unavailable.

For the cantilever-wing configuration, these methods are used to calculate weight and CG contributions from the main wing, the horizontal and vertical stabilizers, the fuselage, and the engines. For unconventional aircraft configurations like the box wing, however, the use of these empirical models can be misleading, given the nature of the data from which these methods are derived. At the same time, calculating a weight and CG that are representative of a competitive box-wing configuration can be difficult without the use of a structural model.

One alternative is to introduce low-fidelity structural approximations for modifying the empirical relationships. However, such a crude approach can detract from the relationship between aerodynamic performance and fuel burn. Instead, the weight of the box-wing configuration is set to be equal to that of the conventional baseline. This is seen as a reasonable choice given that recent efforts $[61,67]$ have suggested that the weight of a box-wing configuration (and in particular, the weight of its wing system) is comparable to that of a similarly-sized conventional design. In addition, setting the lift target equal to that of the cantilever-wing aircraft enables a direct comparison of aerodynamic performance, given that each aircraft is of the same span.

With regard to the CG of the box-wing configuration, the $x$-coordinate is determined by the volume centroid of the wing system, which is allowed to vary throughout the optimization process. In this way, the $\mathrm{CG}$ is tied to the weight distribution of the wing structure and the stored fuel, which are both assumed to be proportional to the volume of the wing system. On the other hand, the $z$-coordinate is determined by a weighted average between the volume centroid of the wing system, and the CG of the remaining aircraft components. It is assumed 
that the ratio between the wing weight and the MTOW is $12 \%$, in accordance to typical values exhibited by conventional aircraft $[61,67]$. The weight of each CF34-10E engine is taken to be approximately $3700 \mathrm{lb}$ [91]. For the cantilever-wing configuration, these engines are mounted at the break station of each wing, whereas for the box-wing configuration, they are aft-mounted on the fuselage. 


\section{Chapter 3}

\section{Results}

\subsection{The Cantilever-Wing Baseline}

\subsubsection{Problem Setup}

The cantilever-wing regional aircraft, herein designated as the CTW100 (which stands for the 100 passenger conventional tube-and-wing aircraft), is used as a performance baseline for comparisons with the box-wing aircraft configuration. As described in Section 2.5, the design of the CTW100 is based on the Embraer E190 regional jet, and has an MTOW of 105,500 lb and an operating empty weight (OEW) of 59,200 lb. For reference, the Embraer E190 has an MTOW and an OEW of 105,359 lb and 61,509 lb, respectively [92]. The CG, which is held fixed for the CTW100, is located $12.32 \mathrm{ft}$ from the leading edge of the main wing root.

For the optimization, only the main wing and the horizontal stabilizer are considered. This includes the carry-through regions of each aerodynamic surface to compensate for the lift that a fuselage would otherwise provide. The fuselage and engines are not modeled given that the primary objective is to evaluate and understand the aerodynamic advantages offered by the wing system of the box-wing configuration, over the conventional cantilever wing. The vertical stabilizer is also not modeled, since its design is based on criteria outside of cruise. Recall that this work assumes that the wing system of the box-wing configuration is compatible with conventional fuselage and empennage designs, as well as podded engines.

However, in order to better represent the performance of the entire aircraft, viscous drag contributions from the fuselage and the vertical stabilizer are included. For the fuselage, the drag coefficient is based on the conventional wing-body-tail geometry of Reist and Zingg [18] and has a value of $C_{D_{\mathrm{F}}}=0.0106$ with a reference area of $S=935 \mathrm{ft}^{2}$. Meanwhile, the drag coefficient of the vertical stabilizer is determined by performing an isolated RANS analysis and has a value of $C_{D_{\mathrm{V}}}=0.0011$ based on the same reference area.

The initial geometry of both the main wing and the horizontal stabilizer are characterized by NACA SC(2)-0012 supercritical symmetric airfoils, with zero twist and angle of attack. This provides a clean slate design that allows equally effective section-shape deformations in either 


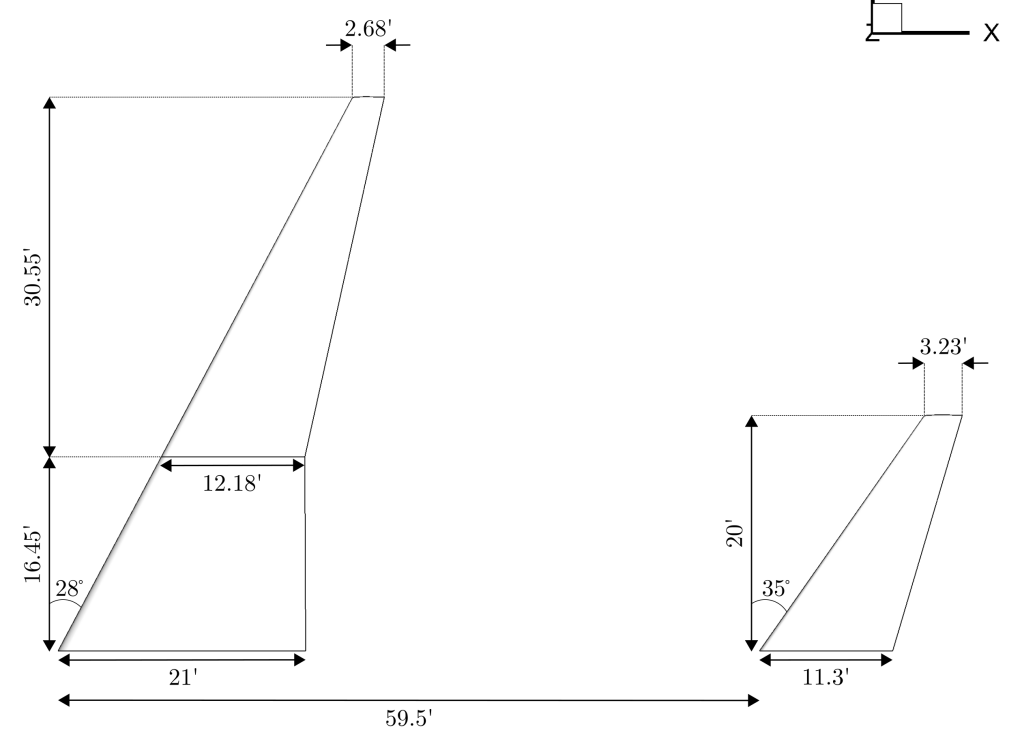

Figure 3.1: CTW100: Initial planform.

Table 3.1: CTW100: Design summary.

\begin{tabular}{rrrrrrrr}
\hline PAX & $\begin{array}{r}b \\
{[\mathrm{ft}]}\end{array}$ & $\begin{array}{r}S \\
{\left[\mathrm{ft}^{2}\right]}\end{array}$ & $\begin{array}{r}\text { AR } \\
{[-]}\end{array}$ & $\begin{array}{r}\text { MAC } \\
{[\mathrm{ft}]}\end{array}$ & $\begin{array}{r}\text { OEW } \\
{[\mathrm{lb}]}\end{array}$ & $\begin{array}{r}\text { MTOW } \\
{[\mathrm{lb}]}\end{array}$ & $\begin{array}{r}\text { Weight* } \\
{[\mathrm{lb}]}\end{array}$ \\
\hline $100+5$ & 94 & 997 & 8.9 & 13.1 & 59,200 & 105,500 & 91,400 \\
\hline
\end{tabular}

* indicates the start of the nominal mission cruise.

direction, while also presenting a reasonable starting point for transonic flow conditions. The initial geometry of the CTW100 is shown in Figure 3.1, with a summary of the initial design provided in Table 3.1 .

The computational domain of the CTW100 is an H-topology structured multi-block mesh, and is shown in Figure 3.2. The mesh is partitioned over 168 blocks and the optimization grid, L0, consists of 2.78 million nodes. Performance is evaluated based on grid converged aerodynamic functionals, which are obtained through Richardson extrapolation assuming second-order convergence. This is done with the addition of two finer grids: L1 and L2, with two and four times the number of grid nodes of L0, respectively. These parameters, as well as the average dimensional and nondimensional off-wall spacings, are summarized in Table 3.2. Note that the average off-wall spacings are in units of mean aerodynamic chord (MAC) and the average $y^{+}$ values are based on the flow solutions of the optimized geometries.

The free-form and axial deformation geometry control system of the CTW100 is shown in Figure 3.3. The aerodynamic surfaces are parameterized with B-splines, and the surface control points are embedded within three FFD volumes: two for the main wing and one for the 


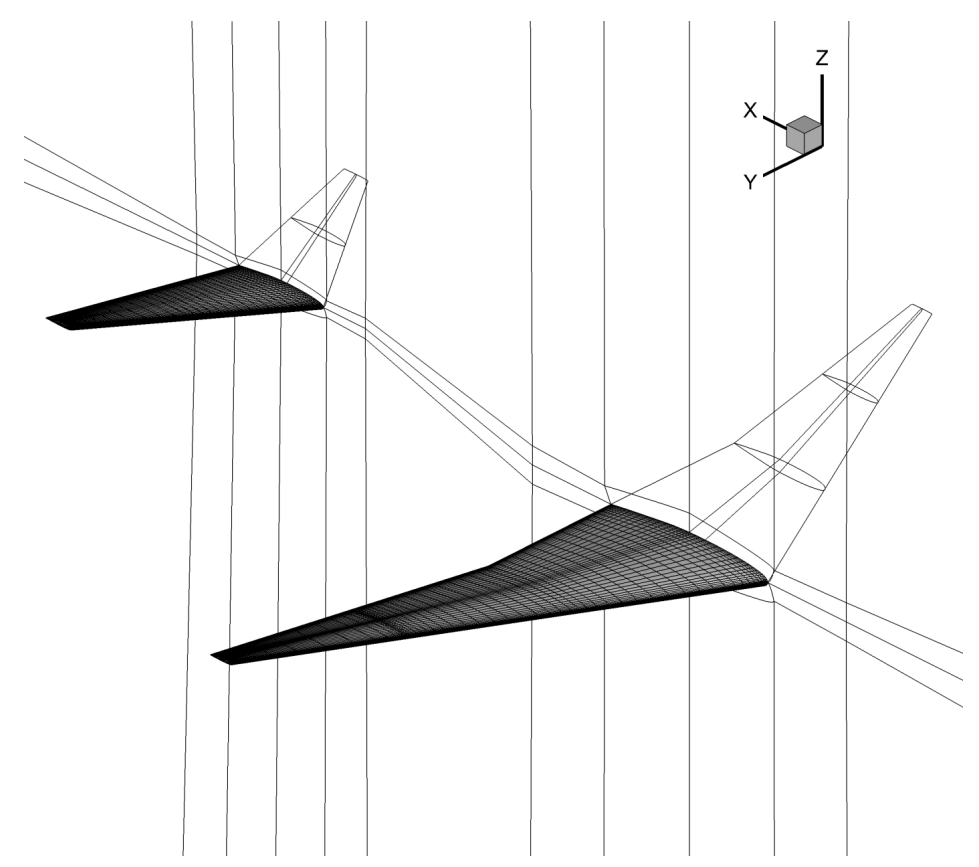

Figure 3.2: CTW100: Surface mesh and blocking topology.

Table 3.2: CTW100: Grid information.

\begin{tabular}{lrrr}
\hline Grid & Number of Nodes & Average Off-Wall Spacing & Average $y^{+}$ \\
\hline L0 & $2.78 \times 10^{6}$ & $1.17 \times 10^{-6}$ & 0.90 \\
L1 & $5.38 \times 10^{6}$ & $8.86 \times 10^{-7}$ & 0.64 \\
L2 & $10.78 \times 10^{6}$ & $6.78 \times 10^{-7}$ & 0.47 \\
\hline
\end{tabular}

Optimization is performed on L0, while L1 and L2 are used in Richardson extrapolation for computing aerodynamic functionals. Average off-wall spacings are in units of MAC.

horizontal stabilizer. The FFD volumes surrounding the main wing consist of 10 FFD-volume cross-sections in total, whereas the FFD volume that embeds the horizontal stabilizer consists of 5 FFD-volume cross-sections. Each FFD-volume cross-section comprises 20 FFD control points, with 10 on either side of a given aerodynamic surface. For the main wing, twist and section shape design variables are active, except at the root FFD-volume cross-section where twist is not permitted; instead a similar functionality is provided by an angle of attack design variable. This setup better ensures that the quality of the mesh is maintained at the symmetry plane. For the horizontal stabilizer, only twist design variables are active, with the intent of maintaining a symmetric control surface. This provides 14 twist degrees of freedom (one for each FFD-volume cross-section, except at the root of the main wing, where it is replaced by the angle of attack design variable), and 200 section shape degrees of freedom (one for each FFD control point surrounding the main wing), for a total of 215 design variables when accounting for the angle of attack degree of freedom. 


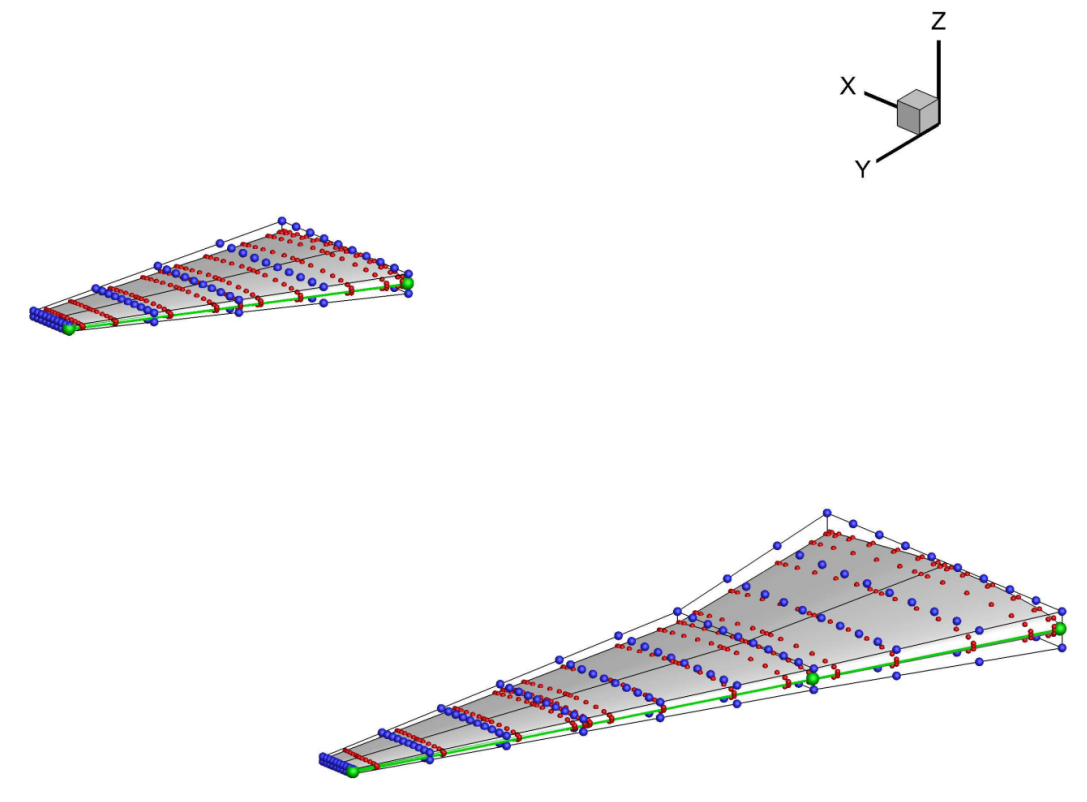

(a) Default view
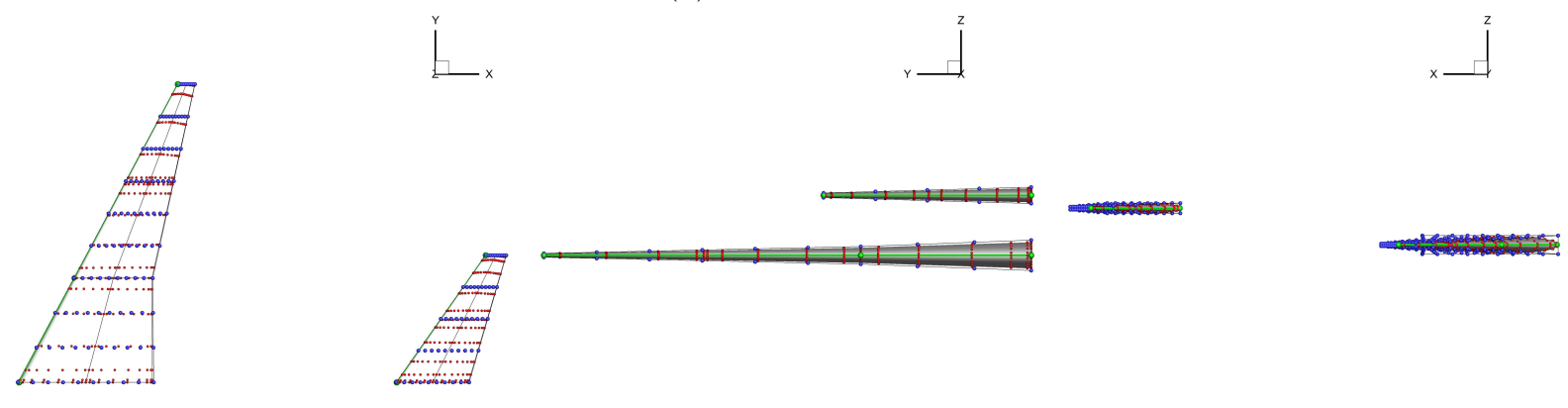

(b) $x y$

(c) $y z$

(d) $x z$

Figure 3.3: CTW100: Free-form and axial deformation geometry control system for aerodynamic shape optimization which includes surface control points (red spheres), FFD volumes (black lines), FFD control points (blue spheres), axial curves (green lines), and axial curve control points (green spheres).

In order to remain consistent with the design of the Embraer E190 regional jet, the CTW100 does not include planform design variables. That is to say, taper, sweep, span, and dihedral design variables are not considered here. However, each FFD-volume is still attached to a linear axial curve at the leading edge, which defines the axis of rotation for the twist design variables. With the omission of taper design variables, the wing planform area is effectively fixed, thus ensuring that it is sufficiently sized for takeoff conditions.

In lieu of structural analysis, minimum thickness-to-chord ratio and minimum wing volume constraints are imposed to prevent the optimizer from designing arbitrarily thin aerodynamic surfaces. In particular, the thickness at any given chordwise station, defined as the distance 
Table 3.3: CTW100: Design variables and geometric constraints.

\begin{tabular}{lccc}
\hline Parameter & Symbol & Lower & Upper \\
\hline \multicolumn{4}{c}{ Design Variables $(215)$} \\
\hline Angle of attack (1) & $\alpha$ & $-8^{\circ}$ & $+8^{\circ}$ \\
Twist (14) & $\theta$ & $-10^{\circ}$ & $+10^{\circ}$ \\
Section shape (200) & $c_{z_{i}}$ & $50 \%$ & $150 \%$ \\
& & \\
\multicolumn{4}{c}{ Geometric Constraints $(104)$} \\
\hline Thickness-to-chord ratio (100) & $t / c$ & $80 \%$ & $\infty$ \\
Volume (1) & $V$ & $1.25 V_{\text {fuel }}$ & $\infty$ \\
Linear twist (3) & - & - & - \\
\hline
\end{tabular}

Bounds given as deviations and percentages are with respect to the initial geometry.

along the local $z$-axis between a pair of FFD control points, is constrained to be no less than 80 percent of its initial value. For example, an airfoil that has an initial thickness-to-chord ratio of 12 percent has a lower bound of 9.6 percent. Meanwhile, the wing volume is constrained to be greater than or equal to 4500 gallons (approximately $600 \mathrm{ft}^{3}$ ) for fuel storage [92], plus 25 percent to accommodate internal wing systems such as slats and flaps. Lastly, to prevent the optimizer from designing wavy aerodynamic surfaces in an attempt to incorporate sectional lift tailoring, a linear twist constraint is imposed across each FFD volume where the twist at each intermediate FFD-volume cross-section is linearly interpolated. An overview of the design variables and geometric constraints involved in the CTW100 optimization is provided in Table 3.3.

\subsubsection{Drag Minimization with Lift and Trim Constraints}

For the aerodynamic design optimization of the CTW100, the objective is to minimize drag subject to lift and trim constraints. More formally, the optimization problem can be summarized as

$$
\begin{aligned}
\operatorname{minimize} & C_{D}, \\
\text { w.r.t. } & \mathbf{v} \\
\text { s.t. } & L=W, \quad C_{M}=0 \\
& \mathcal{C}_{\text {egeo }}=0, \quad \mathcal{C}_{\text {igeo }_{\text {ge }}} \geq 0
\end{aligned}
$$

where $\mathbf{v}=\left[\alpha, \mathbf{v}_{\text {geo }}^{\mathrm{T}}\right]^{\mathrm{T}}$ is the vector of design variables, and $\mathcal{C}_{\text {egeo }}$ and $\mathcal{C}_{\text {igeo }}$ are the vectors of geometric equality and inequality constraints, respectively. $\mathcal{C}_{\text {egeo }}$ includes the linear twist constraints, while $\mathcal{C}_{\text {igeo }}$ consists of the minimum thickness-to-chord ratio and minimum wing volume 


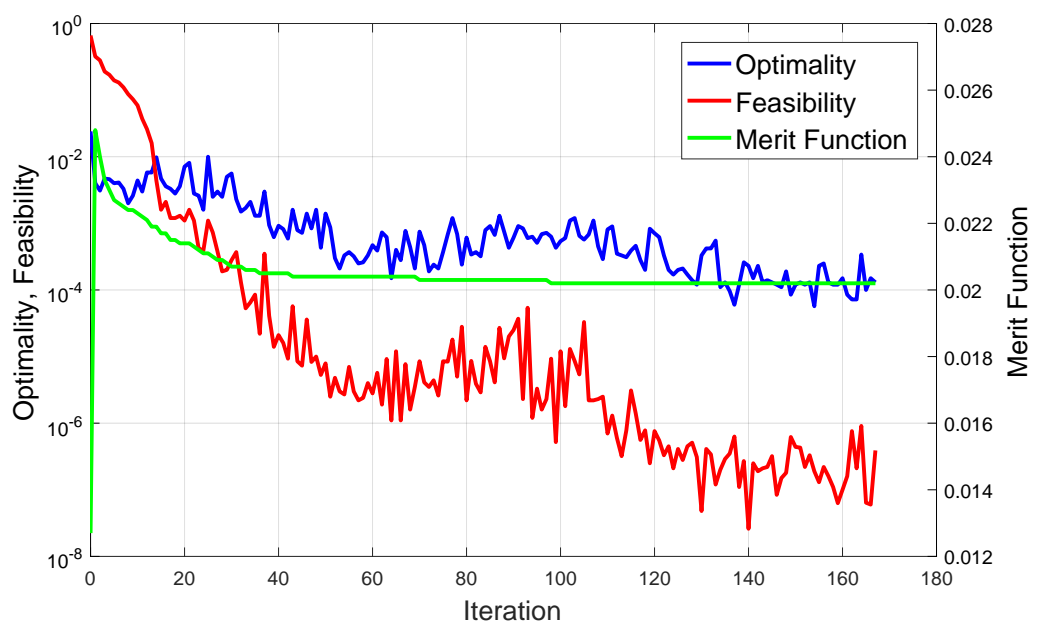

Figure 3.4: CTW100: Optimization history. The Merit function represents $C_{D}$.

constraints, as provided in Table 3.3. A nominal cruise mission is considered at a Mach number of 0.78 and an altitude of $36,000 \mathrm{ft}$, which corresponds to a Reynolds number of $23.6 \times 10^{6}$, based on an MAC of $13.1 \mathrm{ft}$. The maximum weight at cruise is 91,400 lb, as determined from the empirical weight models.

An optimized design was obtained following the completion of 167 design iterations on 168 processors, i.e. as many processors as there are blocks. As shown in Figure 3.4, the end of the optimization is marked by the asymptotic behavior of the Merit function, the convergence of Feasibility to an absolute tolerance of $10^{-6}$, and the reduction in Optimality by roughly two orders of magnitude. With Feasibility satisfied, the Merit function represents the objective function, namely, the drag coefficient. On the optimization grid, the final value of the drag coefficient was found to be 202 counts for the wing-tail geometry.

By the end of the optimization, the minimum bound on thickness-to-chord ratio was reached throughout most of the FFD-volume cross-sections, but without taper design variables, the optimizer was unable to attain a minimum volume design. Nonetheless, the optimized CTW100 has a wing volume of $964 \mathrm{ft}^{3}$, a wing planform area of $997 \mathrm{ft}^{2}$, and a wing-tail wetted area of $1,318 \mathrm{ft}^{2}$. The final CTW100 is characterized by an angle of attack of 6.4 degrees.

As illustrated in Figure 3.5, the optimized design satisfies the lift and trim constraints while maintaining smooth pressure gradients over the upper surface of both the main wing and the horizontal stabilizer. Figure 3.6a shows that for the optimized design, the spanwise lift distribution over the main wing is elliptical in form, with a modest shift towards the wing root as a consequence of the trim constraint. Indeed, with the majority of the outboard region positioned aft of the CG, inboard loading was found to be favorable for counterbalancing the nose-down pitching moment. This was achieved in part through a large twist at the wing root, as indicated by the magnitude of the angle of attack. On the other hand, Figure 3.6b shows that the spanwise lift distribution over the horizontal stabilizer is nearly elliptical, but negative. 

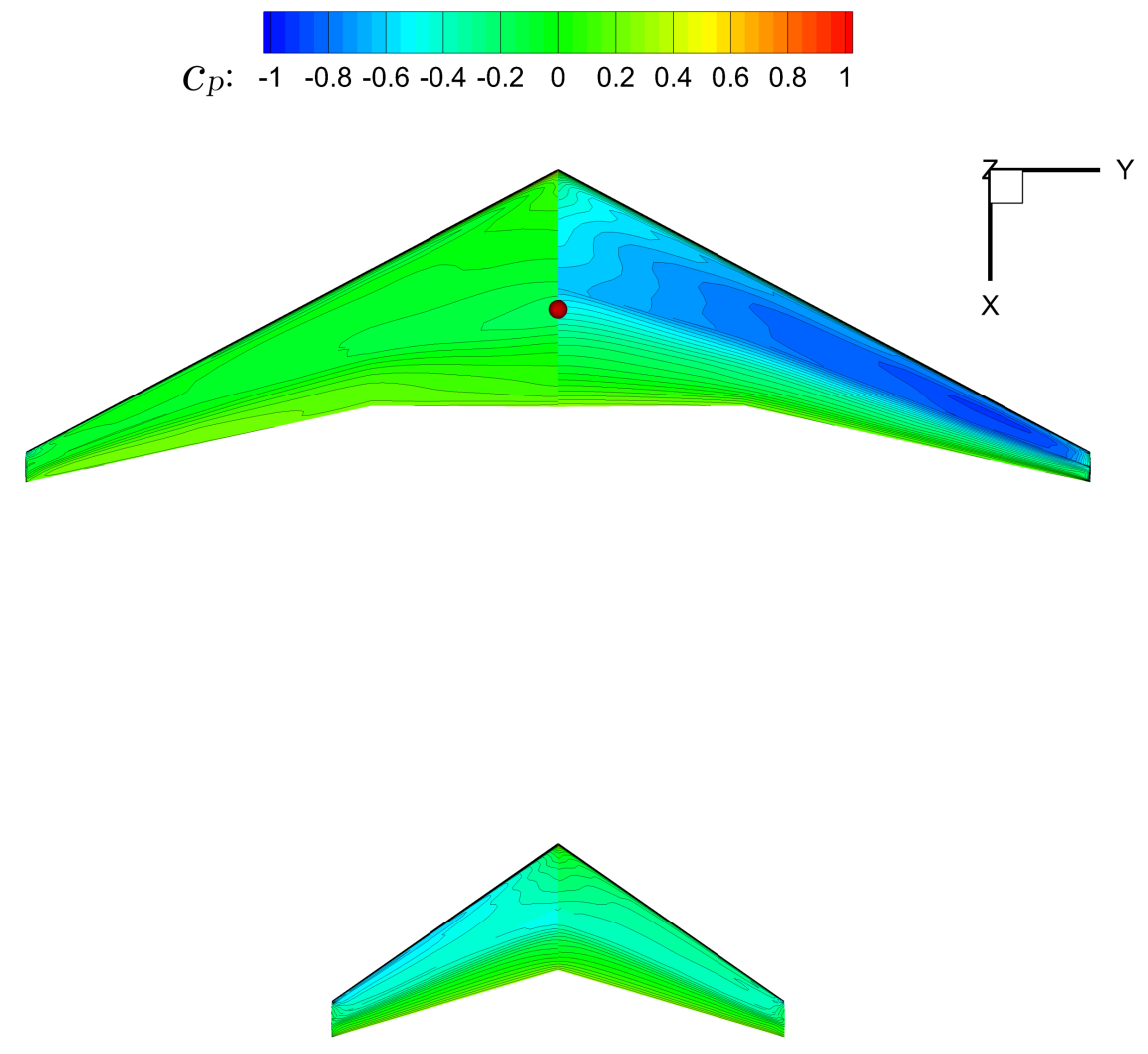

Figure 3.5: CTW100: Surface pressure coefficient contours over the lower (left) and upper (right) surfaces of the optimized wing-tail geometry. The red sphere represents the CG.

This implies that a downforce from the horizontal stabilizer is necessary for maintaining steady level flight, and as a result, constitutes trim drag.

Figure 3.7 illustrates the initial and optimized pressure coefficient distributions and section shapes at six spanwise locations, namely, at the wing root, at $20 \%, 40 \%, 60 \%$, and $80 \%$ of the semispan, and at the wing tip. Here, it can be seen that the optimizer has designed shockfree supercritical cambered airfoils, while introducing wash out, namely, a gradual decrease in incidence angle from the root to the tip. The pressure coefficient distributions exhibit smooth pressure recoveries, which are favorable for maintaining attached boundary layers in viscous flow, in agreement with the findings of Osusky et al. [47]. Note that the depicted airfoils have been rotated by the angle of attack so that the direction of streamwise flow is aligned with the $x$ axis. This way, it becomes clear that although the angle of attack is large, the implementation of an effective negative twist at the wing root (through the section-shape design variables) prevents the formation of a leading-edge pressure spike.

From the grid convergence study, the optimized wing-tail design was found to have a drag coefficient of 164 counts and a lift-to-drag ratio of 27.56. When accounting for the drag contributions from the fuselage $\left(C_{D_{\mathrm{F}}}=0.0100\right)$ and the vertical stabilizer $\left(C_{D_{\mathrm{V}}}=0.0010\right)$, the 


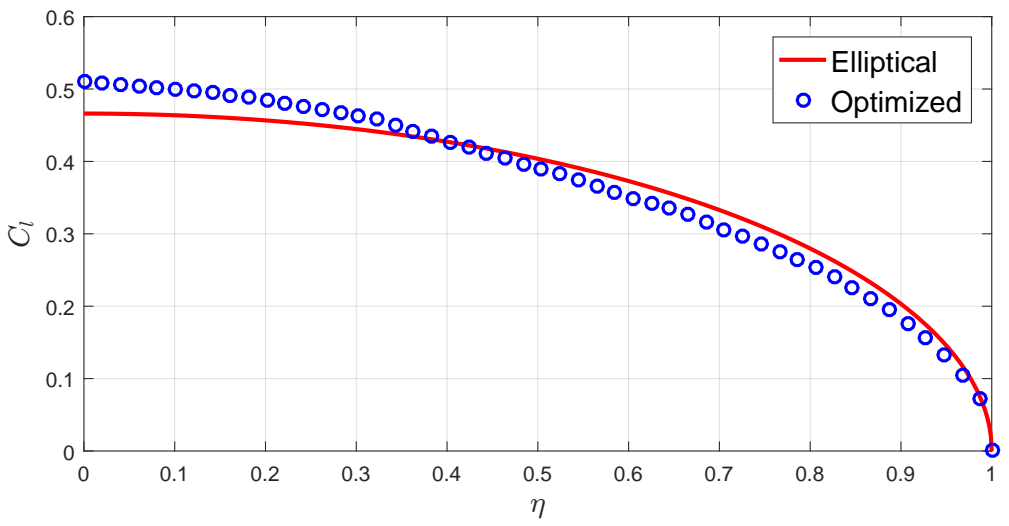

(a) Main wing

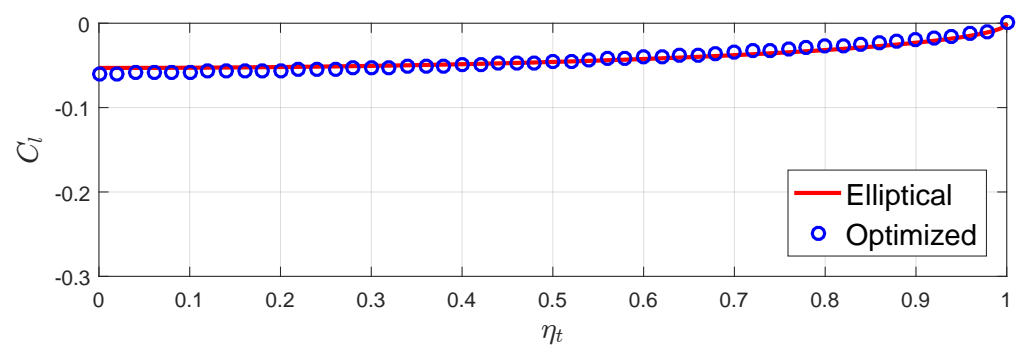

(b) Horizontal stabilizer

Figure 3.6: CTW100: Spanwise lift distribution.

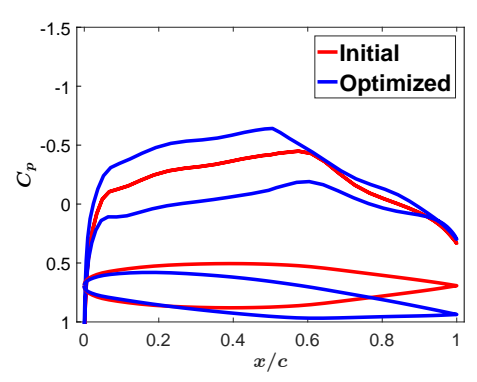

(a) Wing root

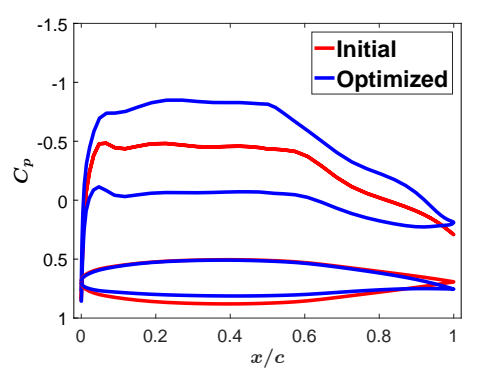

(d) $60 \% \mathrm{span}$

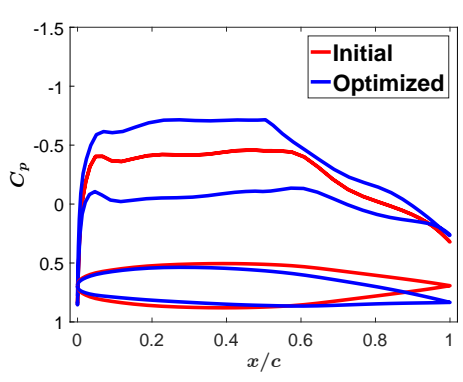

(b) $20 \%$ span

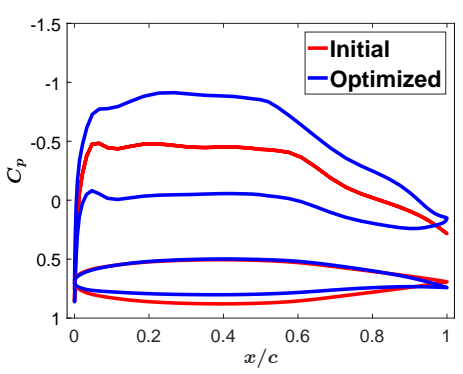

(e) $80 \%$ span

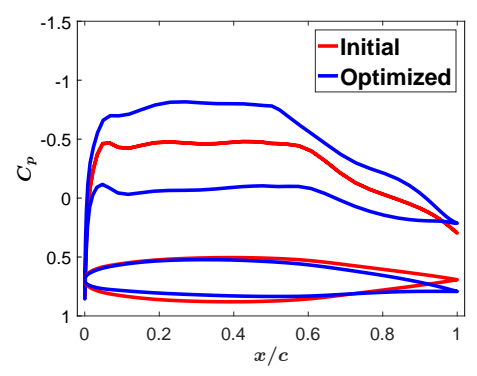

(c) $40 \%$ span

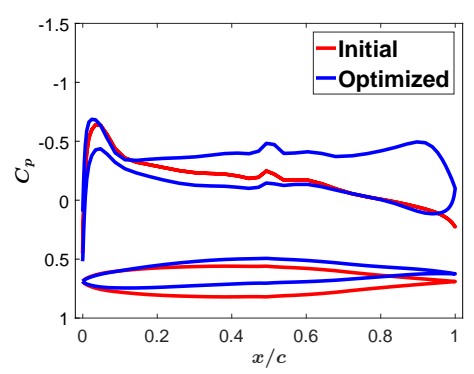

(f) Wing tip

Figure 3.7: CTW100: Pressure coefficient distributions and airfoil profiles. 
Table 3.4: CTW100: Grid convergence study.

\begin{tabular}{rrrrrrrrr}
\hline Grid & Number of Nodes & Average $y^{+}$ & $C_{L}$ & $C_{D_{\mathrm{W}}}$ & $C_{D_{f_{\mathrm{W}}}}$ & $C_{D_{p_{\mathrm{W}}}}$ & $D_{f_{\mathrm{W}}} / D_{p_{\mathrm{W}}}$ & $L / D_{\mathrm{W}}$ \\
\hline L0 & $2.78 \times 10^{6}$ & 0.90 & 0.454 & 0.0202 & 0.0086 & 0.0116 & 0.734 & 22.49 \\
L1 & $5.38 \times 10^{6}$ & 0.64 & 0.454 & 0.0188 & 0.0079 & 0.0109 & 0.719 & 24.16 \\
L2 & $10.78 \times 10^{6}$ & 0.47 & 0.455 & 0.0179 & 0.0073 & 0.0106 & 0.694 & 25.41 \\
\hline
\end{tabular}

$\mathrm{W}$ represents the wing-tail geometry only.

Table 3.5: CTW100: Optimization results.

\begin{tabular}{rrrrrrrrrrrr}
\hline $\begin{array}{r}\text { Weight* } \\
{[\mathrm{lb}]}\end{array}$ & $\begin{array}{r}S \\
{\left[\mathrm{ft}^{2}\right]}\end{array}$ & $\begin{array}{r}S_{\text {wet }} \\
{\left[\mathrm{ft}^{2}\right]}\end{array}$ & $\begin{array}{r}C_{L} \\
{[-]}\end{array}$ & $\begin{array}{r}C_{D_{\mathrm{W}}} \\
{[-]}\end{array}$ & $\begin{array}{r}L / D_{\mathrm{W}} \\
{[-]}\end{array}$ & $\begin{array}{r}C_{D} \\
{[-]}\end{array}$ & $\begin{array}{r}C_{D_{f}} \\
{[-]}\end{array}$ & $\begin{array}{r}C_{D_{p}} \\
{[-]}\end{array}$ & $\begin{array}{r}L / D \\
{[-]}\end{array}$ & $\begin{array}{r}D / q_{\infty} \\
{\left[\mathrm{ft}^{2}\right]}\end{array}$ & $\begin{array}{r}\text { Drag } \\
{[\mathrm{lb}]}\end{array}$ \\
\hline 91,400 & 997 & 1,318 & 0.452 & 0.0164 & 27.56 & 0.0274 & 0.0174 & 0.0100 & 16.50 & 27.40 & 5,541 \\
\hline
\end{tabular}

* indicates the start of the nominal mission cruise and $\mathrm{W}$ represents the wing-tail geometry only.

drag coefficient of the regional aircraft becomes 274 counts, and results in a lift-to-drag ratio of 16.50. Although nearly identical in design, the conventional cantilever-wing regional aircraft of Reist and Zingg [18], which models the fuselage, is characterized by a lift-to-drag ratio of 19.8. This difference can be attributed to the viscous drag of the carry-through regions included in this work, which leads to an overestimate of the total drag when accounting for the fuselage and vertical stabilizer drag approximations. However, this is less of a concern given that consistency is maintained between the aerodynamic design optimization of each aircraft. An overview of the grid convergence study is given in Table 3.4, while a summary of the optimization results is provided in Table 3.5.

\subsection{Exploratory Aerodynamic Shape Optimization of the Box Wing}

\subsubsection{Problem Setup}

For the box-wing aircraft configuration, exploratory aerodynamic shape optimization is first performed with the main purpose of refining the initial planform for the ensuing aerodynamic design optimization, while also providing an opportunity for identifying important trends and trade-offs related to major design features such as sweep, height-to-span ratio, stagger-to-span ratio, planform area, and wetted area. To this end, the optimizer is given significant geometric freedom to explore the design space, and the objective is to maximize the lift-to-drag ratio. Such an objective will drive the optimizer towards a design that can best exemplify the advantages of the box wing, which are aerodynamic in nature. This design is herein designated as the BWE-1.

In order to maximize the freedom given to the optimizer, lift and trim constraints are not included. Without a structural model within the optimization framework, a lift constraint 
would require the wing planform to remain largely unchanged throughout the optimization, given the strong dependence of structural weight on the geometric parameters of the wing. On the other hand, the exclusion of a trim constraint is done to place priority on bringing out the best aerodynamic qualities that the BWE-1 design has to offer, purely through the objective of maximizing the lift-to-drag ratio.

The initial geometry of the BWE-1 is shown in Figure 3.8. It consists of a fore wing, an aft wing, and a vertical tip fin, with the fin connected to the wing tips by blended transitions that aid in reducing interference drag. To remain consistent with the CTW100, the carry-through regions of the fore and aft wings are also included. As a starting point, the initial planform of the fore wing is chosen to be identical to the main wing of the CTW100, while the aft wing has a constant taper with a root chord equal to that of the CTW100 horizontal stabilizer, and a tip chord equal to that of the CTW100 main wing. Although oversized, this initial geometry provides the optimizer more freedom to reshape the design, especially since the upper and lower bounds on the FFD design variables are a function of the initial parameters. To maximize the vertical separation between the fore and aft wings, the root of the fore wing is positioned at the bottom of the fuselage, whereas the root of the aft wing is stationed near the tip of the vertical stabilizer in reference to the Embraer E190 regional jet. The fore and aft wings also have +2 degrees and -2 degrees of dihedral, respectively. This yields an average height-span-ratio of 0.23 and an average stagger-to-span ratio of $0.63 .{ }^{1}$ Since the benefit of wing sweep is symmetric, the fore and aft wings start with +28 degrees of leading-edge sweep and -28 degrees of trailingedge sweep, respectively. This is done to provide each wing an equal opportunity for reaching the same upper and lower bounds on sweep. As with the CTW100, the initial wing geometry (which includes the fore and aft wings, the vertical tip fin, and the blended transitions) has zero twist and angle of attack, and is characterized by NACA SC(2)-0012 supercritical symmetric airfoils.

The computational domain of the BWE-1 is an H-topology structured multi-block mesh, and is shown in Figure 3.9. The mesh is partitioned over 288 blocks and the optimization grid, L0, consists of 5.06 million nodes. For Richardson extrapolation with an assumed second-order convergence, two finer grids are used: L1 and L2, with two and four times the number of grid nodes of L0, respectively. These details, alongside the average off-wall spacings are provided in Table 3.6.

Here it should be noted that the difference in grid size between the CTW100 and the BWE-1 comes from the greater number of blocks associated with the more complicated blocking topology of the BWE-1, alongside the aim of loading each processor with the same number of grid nodes in order to best leverage the available memory of each computer node. At the same time, it is a convenient coincidence that the anticipated flow complexity that is likely to arise from the interaction between the fore and aft wings, and the vertical tip fin will impose a

$1 \quad$ Streamwise stagger is defined along the $x$-axis as the distance from the leading edge of the fore wing root to the leading edge of the aft wing root 


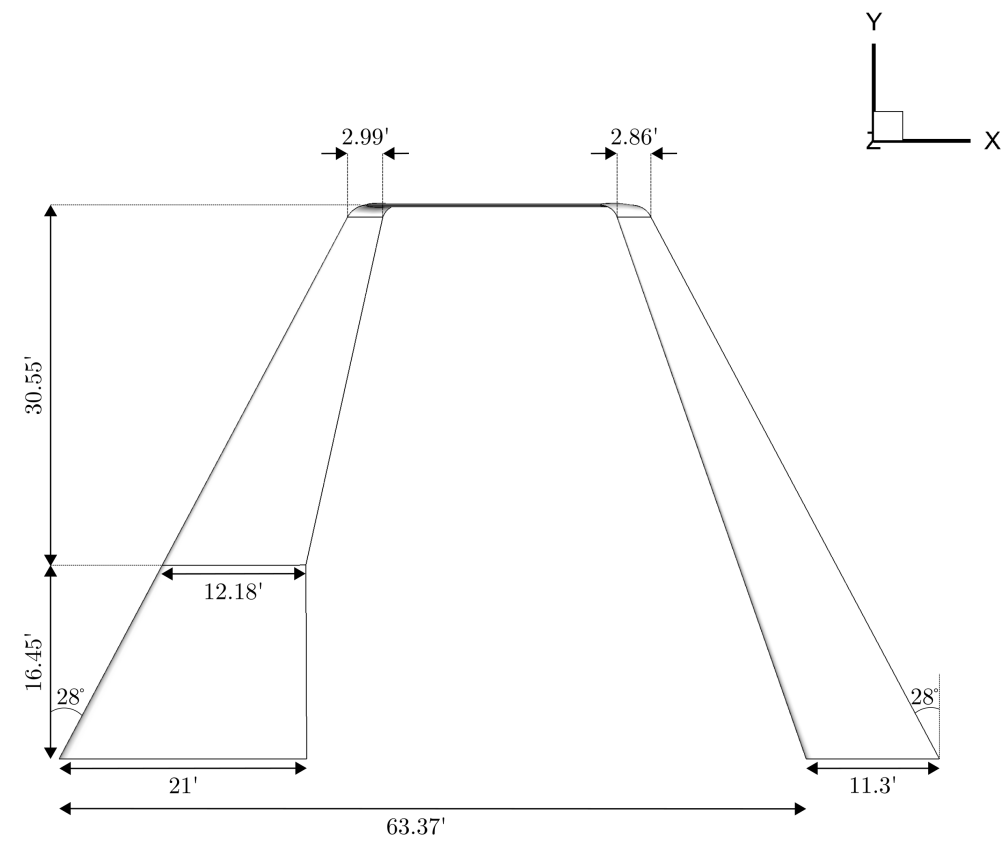

Figure 3.8: BWE-1: Initial planform.

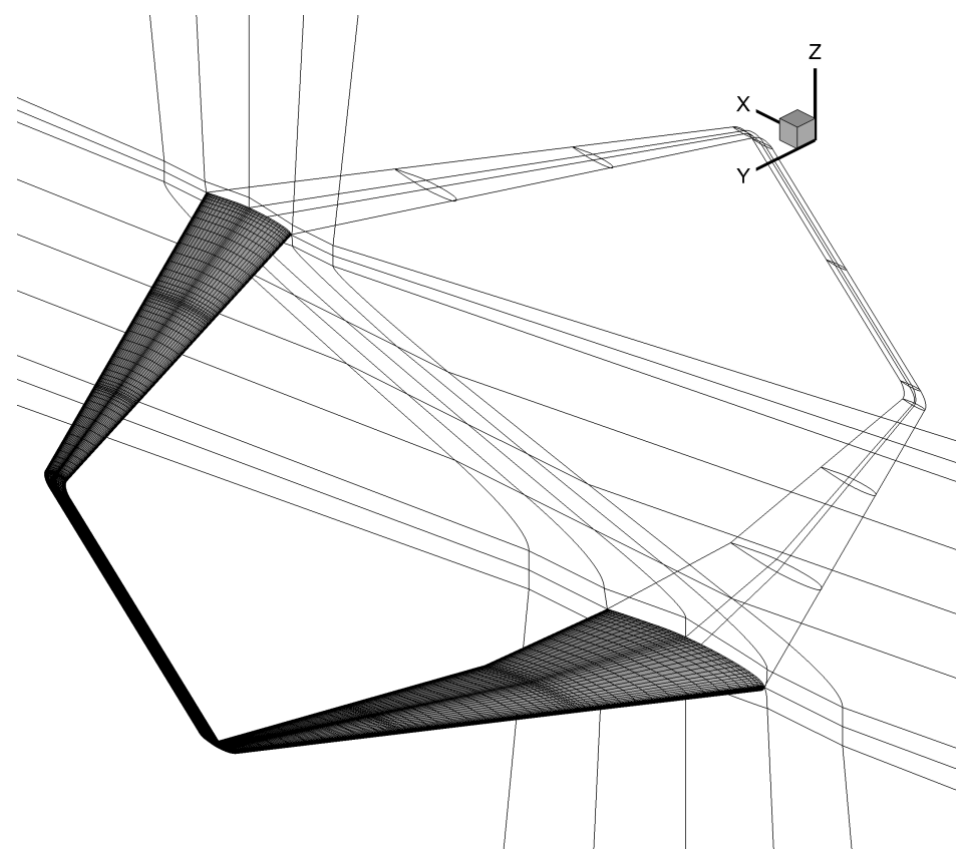

Figure 3.9: BWE-1: Surface mesh and blocking topology.

more stringent meshing requirement. Regardless, for both the CTW100 and the BWE-1, the boundary-layer blocks are nearly the same (i.e. similar average off-wall spacings, number of grid nodes, and grid node distributions), leading to a similar level of boundary-layer resolution.

The free-form and axial deformation geometry control system is shown in Figure 3.10. The aerodynamic surfaces are parameterized with B-splines, and the surface control points are 
Table 3.6: BWE-1: Grid information.

\begin{tabular}{lrrr}
\hline Grid & Number of Nodes & Average Off-Wall Spacing & Average $y^{+}$ \\
\hline L0 & $5.06 \times 10^{6}$ & $1.13 \times 10^{-6}$ & 0.78 \\
L1 & $9.81 \times 10^{6}$ & $8.51 \times 10^{-7}$ & 0.56 \\
L2 & $19.63 \times 10^{6}$ & $6.51 \times 10^{-7}$ & 0.41 \\
\hline
\end{tabular}

Optimization is performed on L0, while L1 and L2 are used in secondorder Richardson extrapolation for computing aerodynamic functionals. Average off-wall spacings are in units of MAC.

embedded within 6 FFD volumes: two for the fore wing, one for the aft wing, one for the vertical tip fin, and one for each blended transition. The FFD volumes surrounding the fore wing, the aft wing, and the vertical tip fin each consist of 10 FFD-volume cross-sections, whereas both of the FFD volumes embedding the blended transitions consist of 5 FFD-volume cross-sections; this makes for a total of 36 unique FFD-volume cross-sections when accounting for overlaps. Each FFD-volume cross-section consists of 20 FFD control points uniformly spaced, with 10 on either side of a given aerodynamic surface. Linear axial curves are positioned at the leading edge of the fore and aft wings, and the vertical tip fin, while cubic axial curves are attached to the leading edges of the two blended transitions; in total, there are 11 axial curve control points, which are positioned at the tip FFD-volume cross-sections of each wing segment, except for the blended transitions where two additional intermediate axial curve control points are necessary for the cubic B-splines.

Overall, the optimization problem involves 807 design variables: an angle of attack degree of freedom, 35 twist degrees of freedom (one for each FFD-volume cross-section except at the root of the fore wing), 36 taper degrees of freedom (one for each FFD-volume cross-section), 720 section shape degrees of freedom (one for each FFD control point), $10 x$-direction degrees of freedom (one for each axial curve control point from 2 to 11), and $5 z$-direction degrees of freedom (one for each axial curve control point from 7 to 11). In conjunction with a number of linear geometric constraints, the $x$ - and $z$-direction degrees of freedom are used to define more meaningful design variables such as leading-edge sweep, height-to-span ratio, and stagger-tospan ratio.

The leading-edge sweep design variables are realized through the $x$-direction degrees of freedom. For the fore wing, the leading-edge sweep is controlled by axial curve control point 3 , with a linear equality geometric constraint imposed on axial curve control points 1,2 , and 3 that force them to remain collinear. On the other hand, the leading-edge sweep of the aft wing is controlled by axial curve control points 10 and 11. In order to have the leading-edge sweep of the vertical tip fin vary in response to changes in the leading-edge sweep of the fore and aft wings, another linear equality geometric constraint, based on a linear interpolation, is imposed on axial curve control points 3 through 10, which also maintains the quality of the blended transitions. The leading-edge sweep of the fore and aft wings are allowed to vary by 


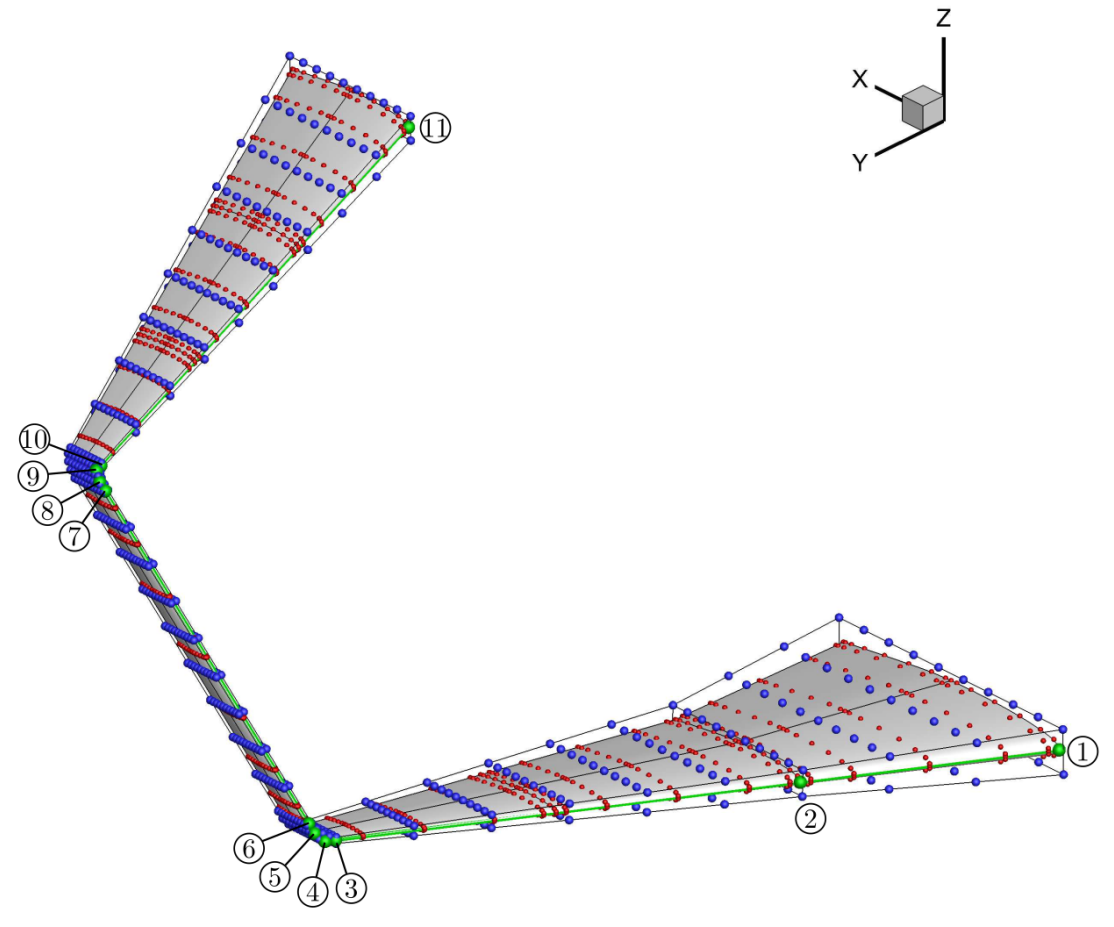

(a) Default view

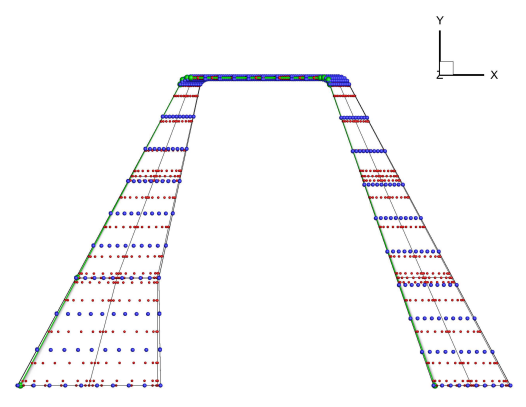

(b) $x y$

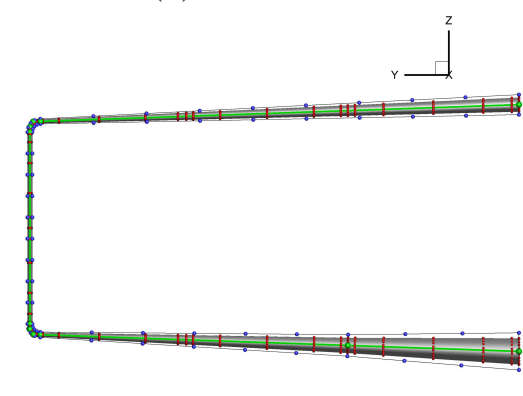

(c) $y z$

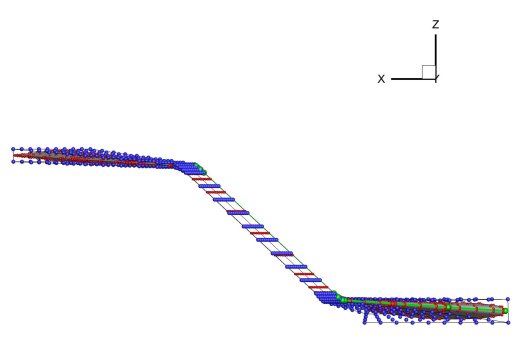

(d) $x z$

Figure 3.10: BWE-1: Free-form and axial deformation geometry control system for aerodynamic shape optimization which includes surface control points (red spheres), FFD volumes (black lines), FFD control points (blue spheres), axial curves (green lines), and axial curve control points (green spheres) [labeled].

\pm 10 degrees in either direction, thus limiting their maximum bounds to wing sweep angles with reasonable structural weight $[60,61,67]$.

Meanwhile, the height-to-span ratio design variable is implemented through geometric constraints on the $z$-direction degrees of freedom. This is done through a series of linear equality geometric constraints on axial curve control points 7 through 11 that force them to translate commensurately. In this work, the height-to-span ratio design variable is defined as an average based on the difference between the $z$-coordinates of axial curve control points 1 and 11 at the 
root of the fore and aft wings, respectively, and the difference between the $z$-coordinates of axial curve control points 3 and 10 at the tip of the fore and aft wings, respectively. The heightto-span ratio design variable is limited to \pm 0.02 (i.e. height bounds of approximately $\pm 2 \mathrm{ft}$ ), which is chosen to keep the root chord of the aft wing close in height to that of the vertical stabilizer, with structural considerations in mind. Following a similar line of reasoning, the stagger-to-span ratio design variable is limited to \pm 0.02 (i.e. stagger bounds of approximately $\pm 2 \mathrm{ft}$ ). This design variable is defined as the streamwise distance between axial curve control points 1 and 11, and is controlled by the $x$-direction degree of freedom of the latter.

Although the freedom provided to both the height-to-span ratio and the stagger-to-span ratio design variables is not substantial, the purpose is to observe, if any, the trade-offs between geometric height and streamwise separation, and the wetted area of the vertical tip fin. In general, these represent trade-offs between induced drag and viscous drag; recall that for the box-wing configuration, an increase to the height-to-span ratio will lead to a decrease in induced drag. Similarly, an increase to the stagger-to-span ratio can reduce the mutual induction effects between the neighboring wings, which also reduces induced drag. However, in both instances, these benefits come with increased wetted area from the vertical tip fin, whose viscous drag will eventually overcome the aerodynamic advantages.

With these definitions, the number of effective design variables becomes 796. As with the CTW100, the thickness-to-chord ratio is constrained to be no less than 80 percent of its initial value at any given chordwise station; the volume of the wing geometry is constrained to be greater than or equal to 4500 gallons or $600 \mathrm{ft}^{3}$, plus 25 percent to account for internal wing systems; and a linear twist constraint is applied across each FFD volume. Note that here it is assumed that fuel can be stored uniformly throughout the wing system.

In addition, a linear taper constraint, similar in definition to the linear twist constraint, is applied across each FFD volume. Together with any linear leading-edge constraints (i.e. the collinearity constraint on the axial curve control points of the fore wing), the linear taper constraint prevents the optimizer from designing nonlinear planforms through trailing-edge variations. This is done for reasons of manufacturability and to keep the design at current technology levels (e.g. for compatibility with conventional flap systems). Although a minimum planform area constraint may also be necessary for ensuring that a sufficient amount of lift can be generated at takeoff, it is anticipated that the planform area will be effectively limited by the minimum taper bounds, the minimum thickness-to-chord ratio constraints, and the minimum wing volume constraint, thus preventing the wing from becoming undersized. Table 3.7 summarizes the effective design variables and geometric constraints for the exploratory aerodynamic shape optimization of the BWE-1.

\subsubsection{Lift-to-Drag Ratio Maximization}

For the exploratory aerodynamic shape optimization of the BWE-1, the objective is to maximize the lift-to-drag ratio, given significant geometric freedom. More formally, the optimization 
Table 3.7: BWE-1: Effective design variables and geometric constraints.

\begin{tabular}{lccc}
\hline Parameter & Symbol & Lower & Upper \\
\hline \multicolumn{4}{c}{ Design Variables $(796)$} \\
\hline Angle of attack (1) & $\alpha$ & $-8^{\circ}$ & $+8^{\circ}$ \\
Twist (35) & $\theta$ & $-10^{\circ}$ & $+10^{\circ}$ \\
Taper (36) & $c_{x z}$ & $50 \%$ & $200 \%$ \\
Section shape (720) & $c_{z_{i}}$ & $50 \%$ & $150 \%$ \\
Leading-edge sweep (2) & $\Lambda$ & $-10^{\circ}$ & $+10^{\circ}$ \\
Height-to-span ratio (1) & $h / b$ & -0.02 & +0.02 \\
Stagger-to-span ratio (1) & $l / b$ & -0.02 & +0.02
\end{tabular}

Geometric Constraints (373)

\begin{tabular}{lccc}
\hline Thickness-to-chord ratio (360) & $t / c$ & $80 \%$ & $\infty$ \\
Volume (1) & $V$ & $1.25 V_{\text {fuel }}$ & $\infty$ \\
Linear twist (6) & - & - & - \\
Linear taper (6) & - & - & - \\
\hline
\end{tabular}

Bounds given as deviations and percentages are with respect to the initial geometry.

problem can be summarized as

$$
\begin{aligned}
\operatorname{maximize} & L / D, \\
\text { w.r.t. } & \mathbf{v}, \\
\text { s.t. } & \mathcal{C}_{\text {egeo }}=0, \quad \mathcal{C}_{\mathrm{i}_{\text {geo }}} \geq 0,
\end{aligned}
$$

where $\mathbf{v}=\left[\alpha, \mathbf{v}_{\text {geo }}^{\mathrm{T}}\right]^{\mathrm{T}}$ is the vector of design variables, and $\mathcal{C}_{\text {egeo }}$ and $\mathcal{C}_{\text {igeo }}$ are the vectors of geometric equality and inequality constraints, respectively. $\mathcal{C}_{\text {egeo }}$ includes the linear twist and taper constraints, while $\mathcal{C}_{\text {igeo }}$ consists of the minimum thickness-to-chord ratio and minimum wing volume constraints, as provided in Table 3.7. Steady level flight (again, without a specified lift target) is considered at a Mach number of 0.78 and an altitude of 36,000 ft, which corresponds to a Reynolds number of $19.78 \times 10^{6}$, based on an MAC of $11.03 \mathrm{ft}$.

An optimized design was obtained following 104 design iterations on 288 processors. As shown in Figure 3.11, the Merit function leveled-off at a lift-to-drag ratio of 25.71 and Optimality reduced by two orders of magnitude. Given that the only nonlinear constraint was the volume constraint which was initially satisfied, the Feasibility remained well below the $10^{-6}$ absolute threshold for the majority of the optimization. Near the end, the optimizer reached the lower bound of the volume constraint and produced an optimal shape that could best leverage the available volume.

In order to reduce wave drag, the optimizer increased the sweep angle of the fore and 


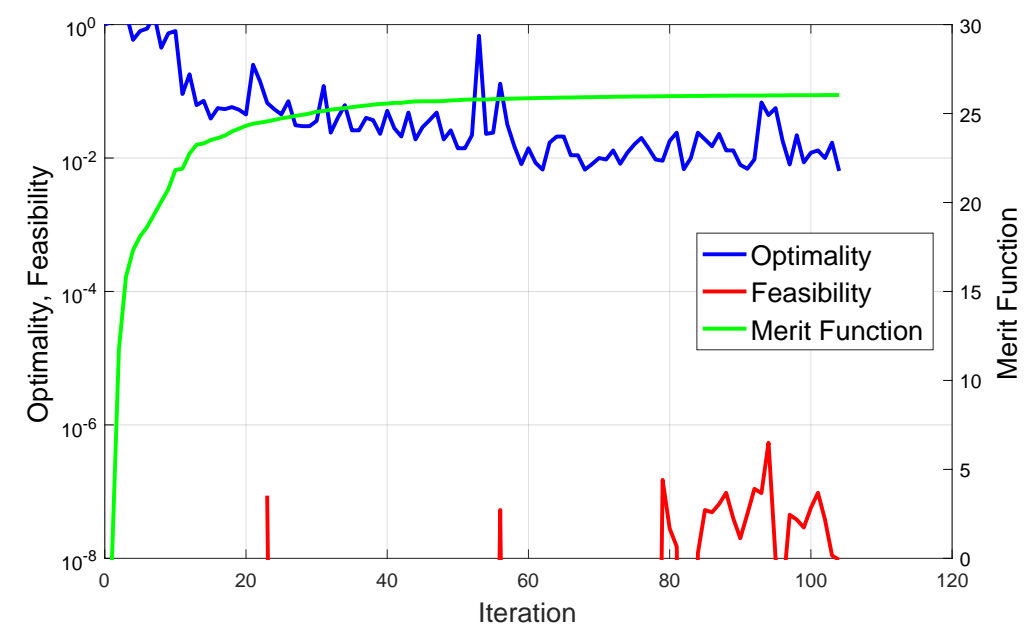

Figure 3.11: BWE-1: Optimization history. The Merit function represents $L / D$.

aft wings to their upper and lower bounds, respectively, namely, by +10 degrees for the fore wing and by -10 degrees for the aft wing. Likewise, the height-to-span and stagger-to-span ratios increased by +0.02 to 0.26 and 0.70 , respectively, or their upper bounds, which agrees with the findings of Andrews and Perez [66]. Interestingly, the optimizer favored allocating planform area to the aft wing over the fore wing, as indicated by a relative planform area of $S_{\mathrm{f}} /\left(S_{\mathrm{f}}+S_{\mathrm{a}}\right)=0.395$. In fact, the taper design variables of the fore wing reached their lower bounds. This is likely a consequence of the asymmetry in mutual induction, because the aft wing imposes less induced drag on the fore wing than the other way around. Here, it remains unclear whether the optimizer preferred to have a fore wing at all.

From the grid convergence study, the lift-to-drag ratio was found to be 32.09. Such a result demonstrates the potential aerodynamic advantage that the box-wing configuration has over the cantilever-wing design. However, in an ensuing drag minimization study in which planform design variables were limited in freedom, and lift and trim constraints were imposed, a number of difficulties were uncovered. Most notable was a large nose-down pitching moment from the large planform area of the aft wing, which was difficult to overcome through twist and section shape design variables alone. For this reason, a second exploratory aerodynamic shape optimization study is performed with the addition of a trim constraint.

\subsubsection{Lift-to-Drag Ratio Maximization with a Trim Constraint}

In order to address the difficulties uncovered in the previous study, a second iteration of the exploratory aerodynamic shape optimization is performed for the box-wing aircraft configuration, herein designated as the BWE-2. Again, the objective is to maximize the lift-to-drag ratio with similar design variables as before, but with the addition of a trim constraint. Recall from Section 2.6 that the CG is determined based on the volume centroid of the wing geometry. This is done by first defining the $x$ - and $z$-coordinates of the CG as design variables; a nonlinear 
constraint is then imposed which dictates that the $x$-coordinate of the CG be positioned at the volume centroid of the wing geometry, and that the $z$-coordinate of the CG be positioned based on a weighted average of the $z$-coordinate of the wing volume centroid and the remaining aircraft components, i.e. the fuselage, the engines, and the vertical stabilizer. In this way, the effect of the CG (and therefore the effect of the distribution of the total volume over the fore wing, the aft wing, and the vertical tip fin) on aerodynamic performance is made transparent to the optimizer, thus allowing the volume distribution of the wing system to be traded with any competing parameters. The optimization problem can be summarized as

$$
\begin{aligned}
\operatorname{maximize} & L / D, \\
\text { w.r.t. } & \mathbf{v} \\
\text { s.t. } & C_{M}=0, \\
& \mathcal{C}_{\text {egeo }_{\text {geo }}}=0, \quad \mathcal{C}_{\mathrm{i}_{\text {geo }}} \geq 0,
\end{aligned}
$$

where $\mathbf{v}=\left[\alpha, x_{\mathrm{CG}}, z_{\mathrm{CG}}, \mathbf{v}_{\text {geo }}^{\mathrm{T}}\right]^{\mathrm{T}}$ is the vector of design variables, and $\mathcal{C}_{\text {egeo }}$ and $\mathcal{C}_{\text {igeo }}$ are the vectors of geometric equality and inequality constraints, respectively. $\mathcal{C}_{\text {egeo }}$ includes the linear twist and taper constraints, and the volume centroid CG constraints, while $\mathcal{C}_{\text {igeo }}$ consists of the minimum thickness-to-chord ratio and minimum wing volume constraints.

With the benefit of hindsight, the previous design was also found to require either a vertical stabilizer with a high forward sweep, or a highly forward-shifted vertical stabilizer when accounting for the CG of the entire aircraft. Although these designs can be achieved in practice, a significantly reduced vertical stabilizer moment arm would necessitate a proportional increase in vertical stabilizer planform area, thus violating the assumption of similar vertical stabilizers between the box-wing and cantilever-wing aircraft configurations. For this reason, the upper and lower bounds on the stagger-to-span ratio design variable are now relaxed to encourage the optimizer to design a more feasible wing geometry. In particular, the stagger-to-span ratio is now permitted to change by \pm 0.2 (i.e. stagger bounds of approximately $\pm 20 \mathrm{ft}$ ). An updated summary of the effective design variables and geometric constraints is provided in Table 3.8.

In total, 117 design iterations were completed on 288 processors. Figure 3.12 illustrates the optimization history. At the end of the optimization, Feasibility is satisfied to an absolute tolerance of approximately $10^{-6}$, and Optimality reduced by more than two orders of magnitude. The Merit function, or the lift-to-drag ratio, converged to a value of 25.96 on the optimization grid. From the grid convergence study, the lift-to-drag ratio of the wing geometry was found to be 32.09 .

The optimized geometry has a height-to-span ratio of 0.26 and a stagger-to-span ratio of 0.78. The former reached its upper bound while the latter settled on an intermediate value. The sweep design variables of the fore and aft wings reached their upper and lower bounds, respectively, namely, +10 degrees for the fore wing and -10 degrees for the aft wing. The trim constraint led to a design with an evenly distributed planform area between the fore and aft 
Table 3.8: BWE-2: Effective design variables and geometric constraints.

\begin{tabular}{lccc}
\hline Parameter & Symbol & Lower & Upper \\
\hline & Design Variables $(798)$ & \\
\hline Angle of attack (1) & $\alpha$ & $-8^{\circ}$ & $+8^{\circ}$ \\
Twist (35) & $\theta$ & $-10^{\circ}$ & $+10^{\circ}$ \\
Taper (36) & $c_{x z}$ & $50 \%$ & $200 \%$ \\
Section shape (720) & $c_{z_{i}}$ & $50 \%$ & $150 \%$ \\
Leading-edge sweep (2) & $\Lambda$ & $-10^{\circ}$ & $+10^{\circ}$ \\
Height-to-span ratio (1) & $h / b$ & -0.02 & +0.02 \\
Stagger-to-span ratio (1) & $l / b$ & -0.2 & +0.2 \\
Streamwise CG (1) & $x_{\mathrm{CG}}$ & $-\infty$ & $+\infty$ \\
Vertical CG (1) & $z_{\mathrm{CG}}$ & $-\infty$ & $+\infty$ \\
& & & \\
& Geometric Constraints & $(375)$ & $\infty$ \\
\hline Thickness-to-chord ratio (360) & $t / c$ & $80 \%$ & - \\
Volume (1) & $V$ & $1.25 V_{\text {fuel }}$ & - \\
Linear twist (6) & - & - & $\bar{x}_{V}$ \\
Linear taper (6) & - & - & $\infty$ \\
Streamwise CG (1) & $x_{\mathrm{CG}}$ & $\bar{x}_{V}$ & $0.12 \bar{z}_{V}+0.88 \bar{z}_{\mathrm{AC}}$ \\
Vertical CG (1) & $z_{\mathrm{CG}}$ & $0.12 \bar{z}_{V}+0.88 \bar{z}_{\mathrm{AC}}$ & \\
\hline
\end{tabular}

Bounds given as deviations and percentages are with respect to the initial geometry.

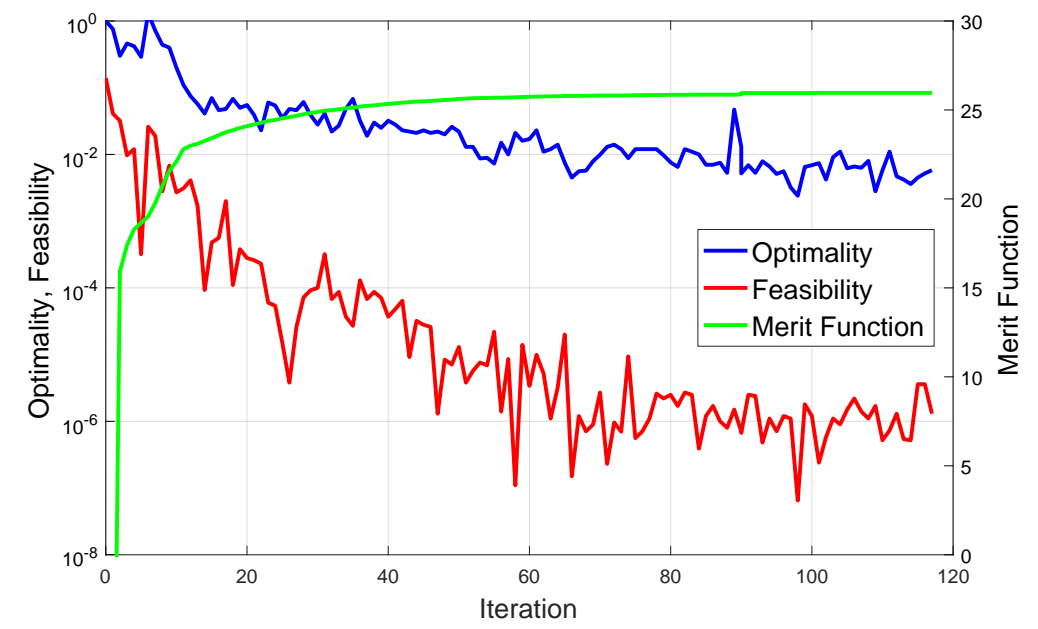

Figure 3.12: BWE-2: Optimization history. The Merit function represents $L / D$. 
wings; the relative planform area was found to be $S_{\mathrm{f}} /\left(S_{\mathrm{f}}+S_{\mathrm{a}}\right)=0.46$. Furthermore, the chord lengths at axial curve control points $1,2,3,10$, and 11 did not reach their upper or lower bounds, but instead settled on an intermediate value. However, the chord lengths at axial curve control points 6 and 7 reduced to their minimum. This suggests that the vertical tip fin is seen as either unnecessary by the optimizer or that only a modest butterfly-shaped sideforce distribution is required to complete the closed-loop circulation pattern. Nonetheless, the optimizer achieved a minimum volume design. The optimized geometry has a planform area of $1,229 \mathrm{ft}^{2}$ and a wetted area of $1,320 \mathrm{ft}^{2}$, namely, a $26 \%$ and a $27 \%$ reduction from the initial geometry, respectively. Relative to the CTW100, the BWE-2 has $23 \%$ more wing planform area, thus confirming that the minimum geometric bounds were sufficient for ensuring that the wing was not undersized for takeoff conditions. The optimized BWE-2 is designed for an angle of attack of -0.17 degrees.

Figure 3.13a illustrates the pressure coefficient contours over the upper surface of the initial and optimized designs. By increasing the sweep and reducing the thickness of the wings, the optimizer achieved a design that experiences little to no wave drag, as indicated by the smooth pressure variations. Figure 3.13b and Figure 3.13c show the inboard and outboard surface pressure coefficient contours of the optimized design, respectively. From these, it can be seen that the suction side of the surface pressure coefficient distribution continues from the upper surface of the fore wing up the inboard surface of the vertical tip fin until the midspan, at which point the suction side continues up the outboard surface of the vertical tip fin, and proceeds along the upper surface of the aft wing. This forms the closed-loop circulation pattern shown in Figure 3.14, which resembles the pattern predicted by linear aerodynamic theory. Given that the sectional force coefficient over the vertical tip fin remains small near the ends, it can be argued that the box wing only requires a small vertical tip fin to complete the closed-loop circulation.

The CG is represented by the red sphere in Figure 3.13a, which is located $43.20 \mathrm{ft}$ and $2.79 \mathrm{ft}$ from the leading edge of the fore wing root along the $x$ - and $z$-axes, respectively. It is apparent that the CG has a slight bias towards the aft wing, as indicated by the relative planform area, but also due to a minor difference in wing volume. With regard to the latter, the section shapes near the root of the aft wing are relatively thick to account for the negative induced flow curvature of the fore wing, as noted by Wolkovitch [22].

Having observed the expected aerodynamic characteristics of the box-wing aircraft configuration, the optimized BWE-2 is used as a basis for the initial geometry of the following aerodynamic design optimization. From here, planform degrees of freedom such as sweep, height-to-span ratio, and stagger-to-span ratio will no longer be included since their maximum bounds, which were based on structural considerations, have already been exhausted. However, taper degrees of freedom will still be included, albeit with less freedom, to allow the design to be adjusted for the correct lift coefficient and altitude. 


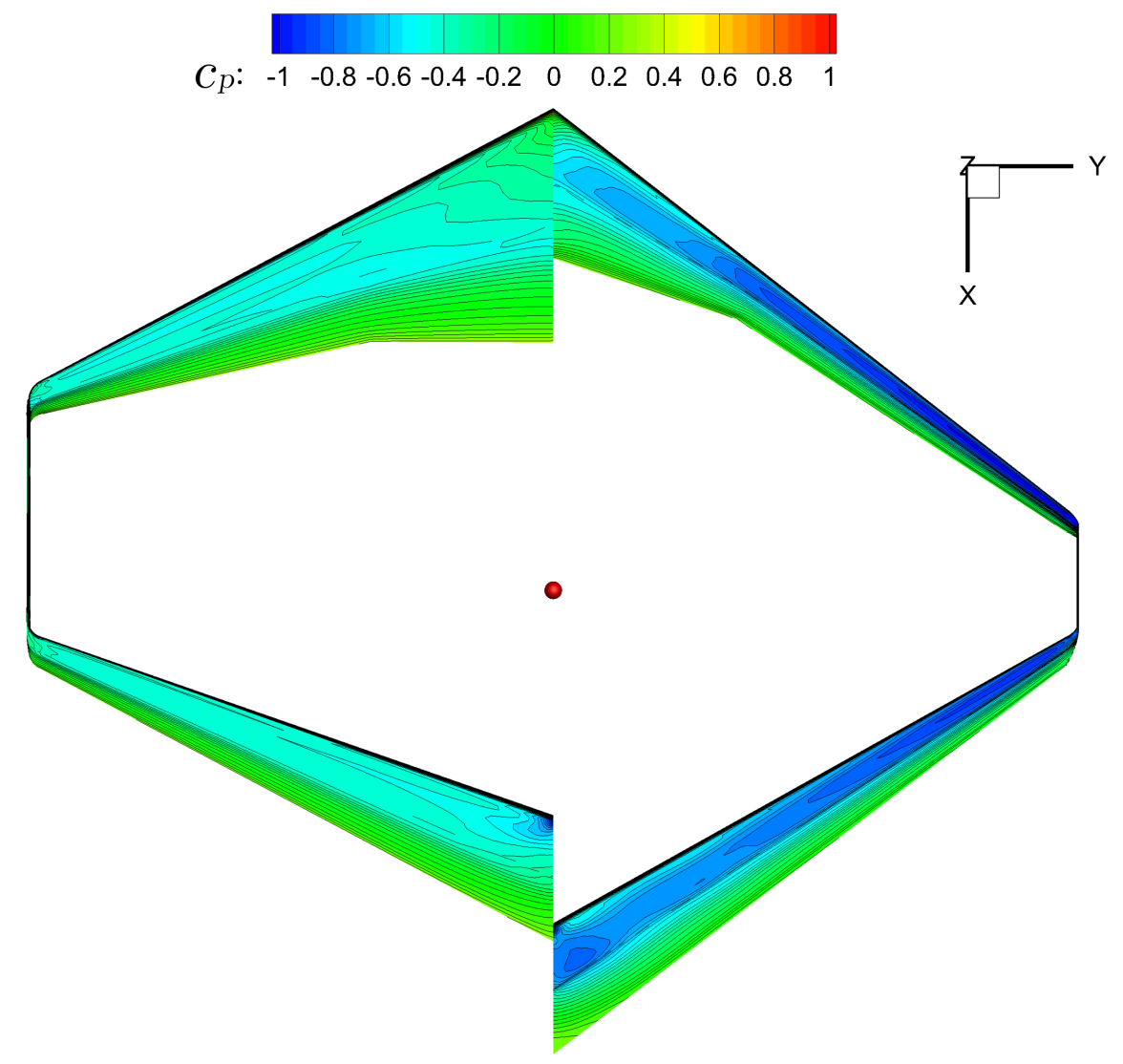

(a) Top view

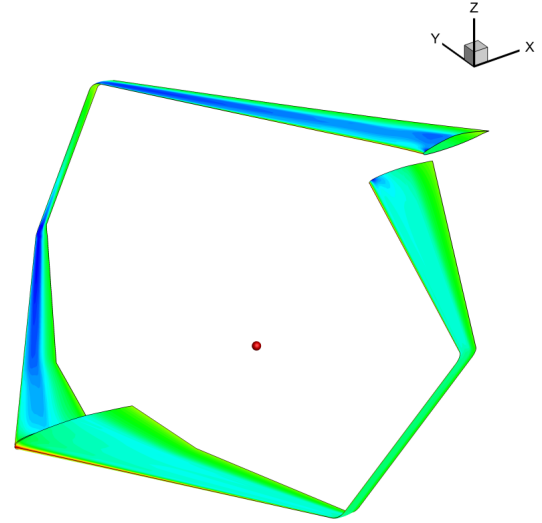

(b) Inboard view

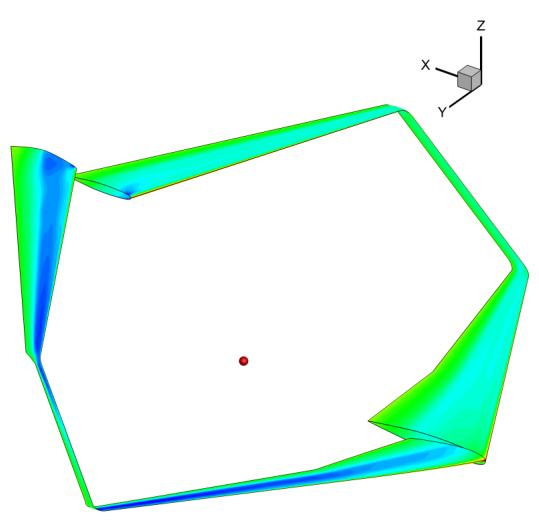

(c) Outboard view

Figure 3.13: BWE-2: Surface pressure coefficient contours for the initial [left, (a)] and optimized [right, (a)] wing only geometries. Inboard and outboard views illustrate the signature closedloop circulation pattern of the box wing. The CG of the optimized design is represented by the red sphere. 

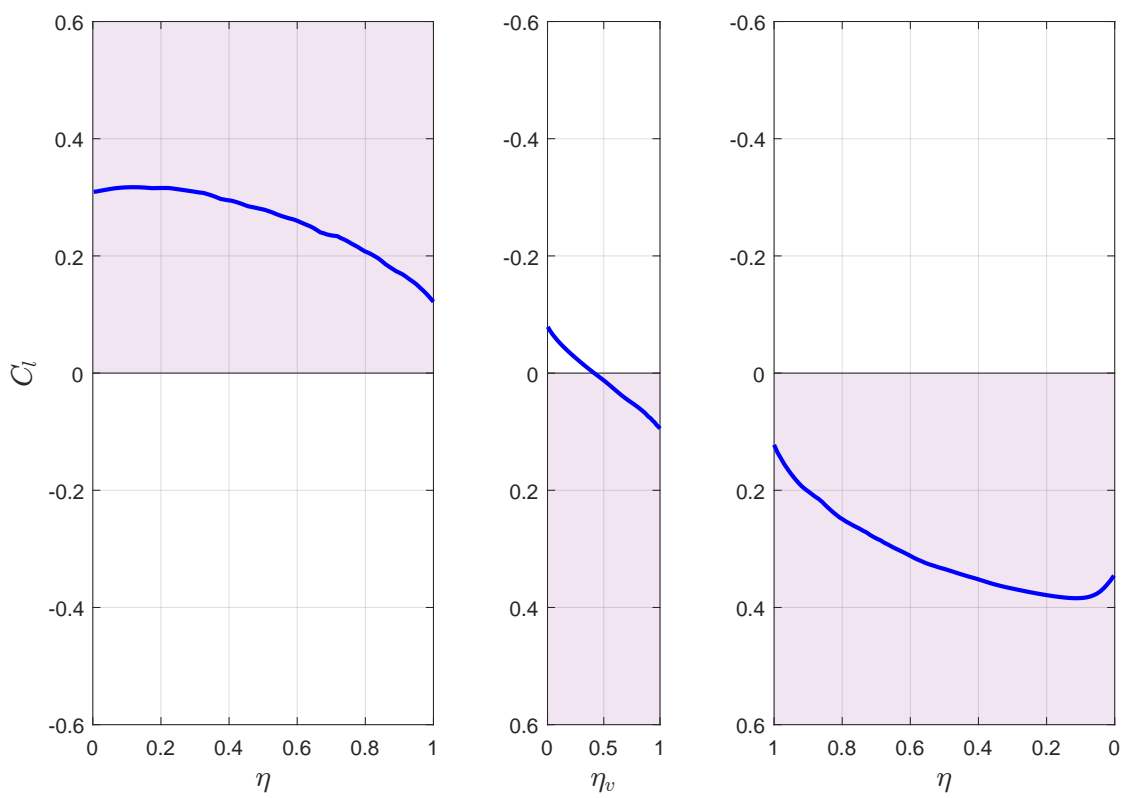

Figure 3.14: BWE-2: Force distributions over the fore wing (left), the vertical tip fin (middle), and the aft wing (right).

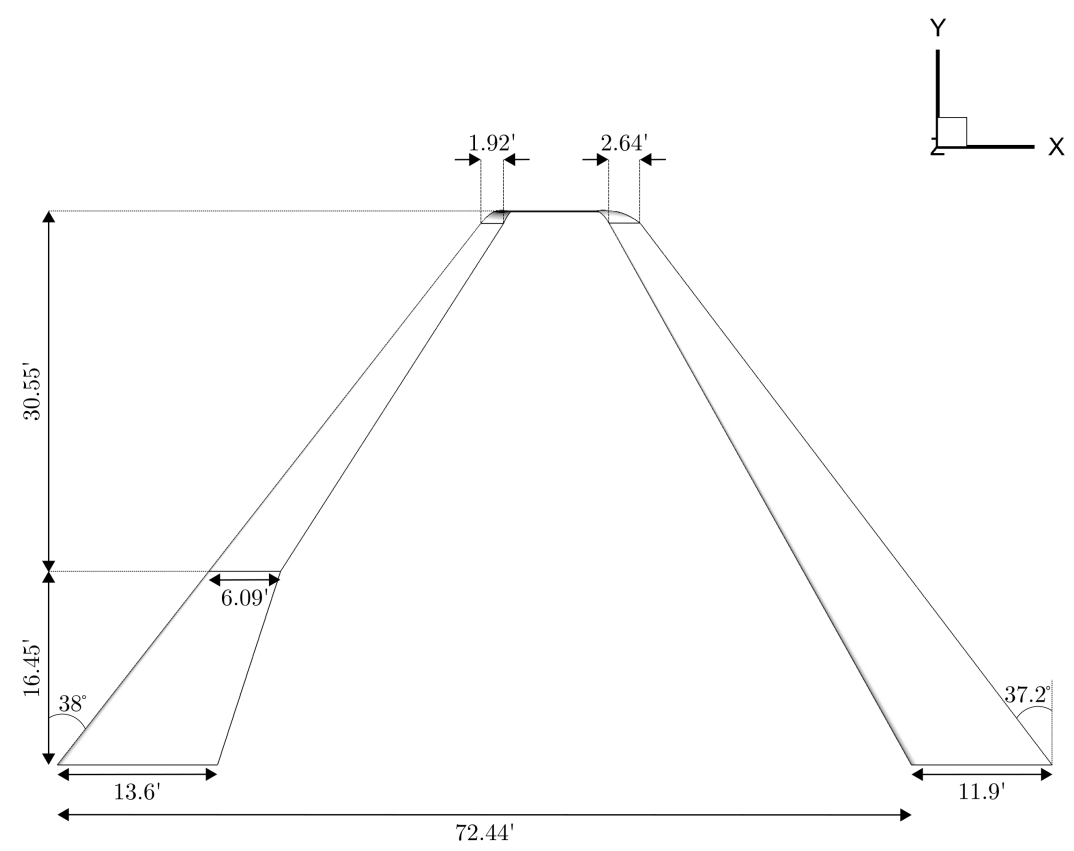

Figure 3.15: BW100: Initial planform. 


\subsection{Aerodynamic Design Optimization of the Box Wing}

\subsubsection{Problem Setup}

High-fidelity optimization is now applied to the aerodynamic design of a box-wing regional aircraft, herein designated as the BW100, in order to evaluate its potential to provide major savings in fuel burn relative to the CTW100. The initial geometry is based on the optimized BWE-2, and is shown in Figure 3.15. Once again, the carry-through regions of each aerodynamic surface are included. The initial wing geometry is characterized by NACA SC(2)-0012 supercritical symmetric airfoils, with zero twist and angle of attack. A summary of the initial design is provided in Table 3.9. The aerodynamic surfaces are parameterized with B-splines, and the surface control points are embedded within the free-form and axial deformation geometry control system depicted in Figure 3.16.

The computational domain of the BW100 is identical to that of the BWE-1 and BWE-2. It is an H-topology structured multi-block mesh and is shown in Figure 3.17. The mesh is partitioned over 288 blocks, and the optimization grid, L0, consists of 5.06 million nodes. For Richardson extrapolation with an assumed second-order convergence, two finer grids are used: L1 and L2, with two and four times the number of grid nodes of L0, respectively. These details, alongside the average off-wall spacings are provided in Table 3.10.

For the BW100, planform design variables such as the height-to-span ratio, the stagger-tospan ratio, and the leading-edge sweep are held fixed. However, taper design variables are still active for the fore and aft wings, and are permitted to vary by $\pm 10 \%$ at a given FFD-volume cross-section. This is done to allow the optimizer the freedom to adjust the relative planform area for a lift coefficient that is effectively lower than that of the BWE-2 optimization; recall that for the BWE-2 optimization, the objective was to maximize the lift-to-drag ratio, thus permitting a higher lift coefficient to be attained. In total, there are 794 design variables: an angle of attack degree of freedom, 35 twist degrees of freedom (one for each FFD-volume cross-section except at the root of the fore wing), 36 taper degrees of freedom (one for each FFD-volume cross-section), 720 section shape degrees of freedom (one for each FFD control point), and two CG degrees of freedom (the $x$ - and $z$-coordinates).

As with the BWE-2 optimization, the thickness-to-chord ratio is constrained to be no less than 80 percent of its initial value at a given chordwise station; the volume of the wing geometry is constrained to be greater than or equal to 4500 gallons or $600 \mathrm{ft}^{3}$, plus 25 percent to account for internal wing systems; and both linear twist and linear taper constraints are applied across each FFD volume. A summary of the design variables and geometric constraints is provided in Table 3.11 . 
Table 3.9: BW100: Design summary.

\begin{tabular}{rrrrrrrr}
\hline PAX & $\begin{array}{r}S \\
{[\mathrm{ft}]}\end{array}$ & $\begin{array}{r}S \\
{\left[\mathrm{ft}^{2}\right]}\end{array}$ & $\begin{array}{r}\text { AR } \\
{[-]}\end{array}$ & $\begin{array}{r}\text { MAC } \\
{[\mathrm{ft}]}\end{array}$ & $\begin{array}{r}\text { OEW } \\
{[\mathrm{lb}]}\end{array}$ & $\begin{array}{r}\text { MTOW } \\
{[\mathrm{lb}]}\end{array}$ & $\begin{array}{r}\text { Weight* } \\
{[\mathrm{lb}]}\end{array}$ \\
\hline $100+5$ & 94 & 1,203 & 7.34 & 8.05 & 59,200 & 105,500 & 91,400 \\
\hline
\end{tabular}

* indicates the start of the nominal mission cruise.

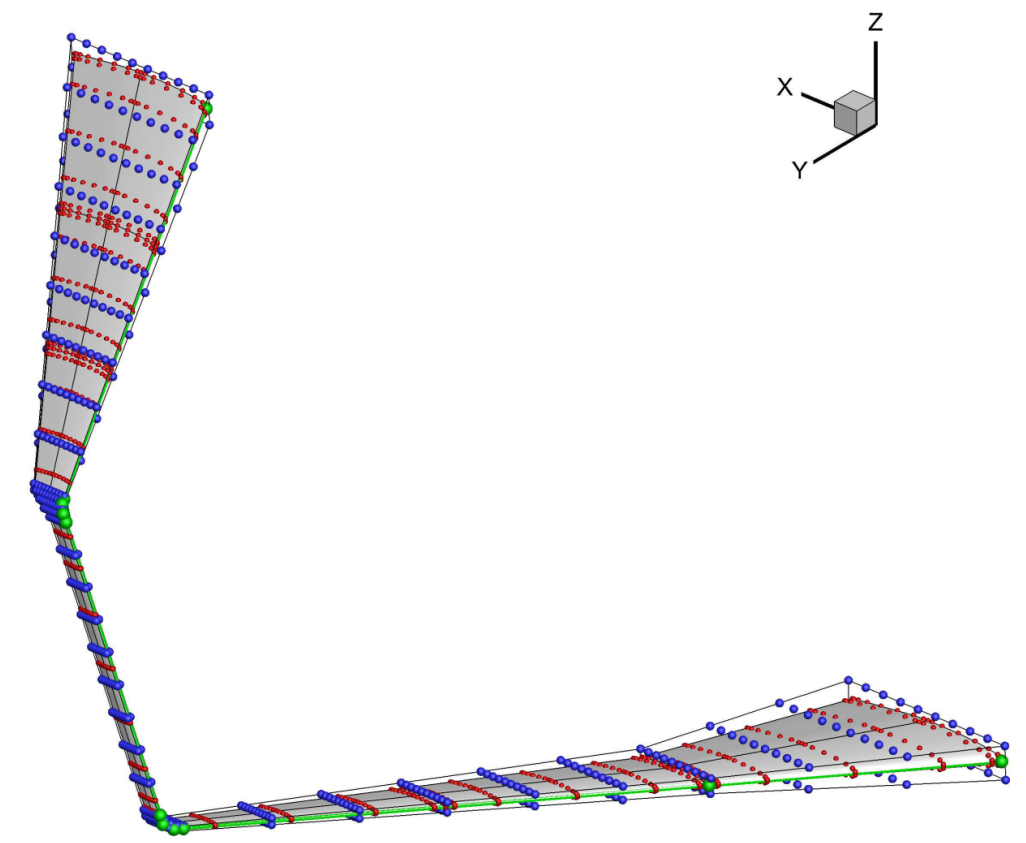

(a) Default view

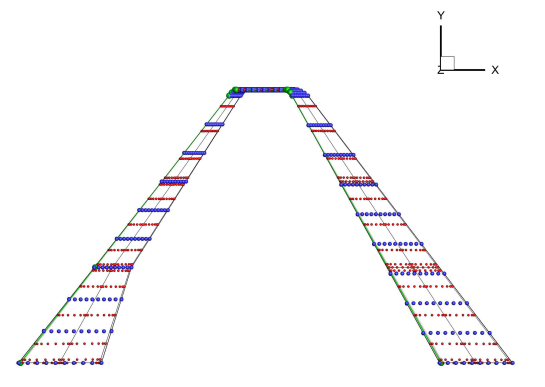

(b) $x y$

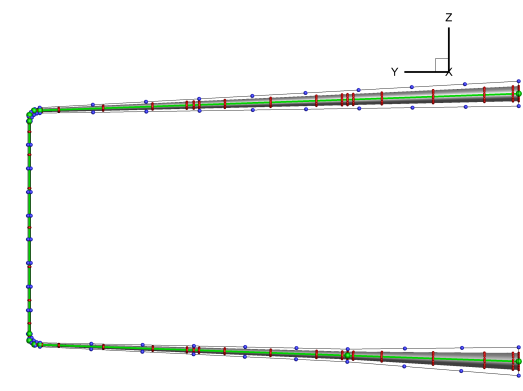

(c) $y z$

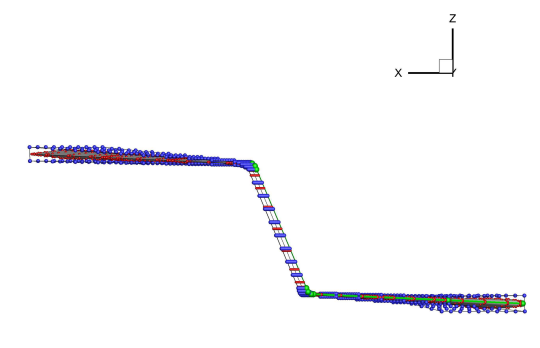

(d) $x z$

Figure 3.16: BW100: Free-form and axial deformation geometry control system for aerodynamic shape optimization which includes surface control points (red spheres), FFD volumes (black lines), FFD control points (blue spheres), axial curves (green lines), and axial curve control points (green spheres). 


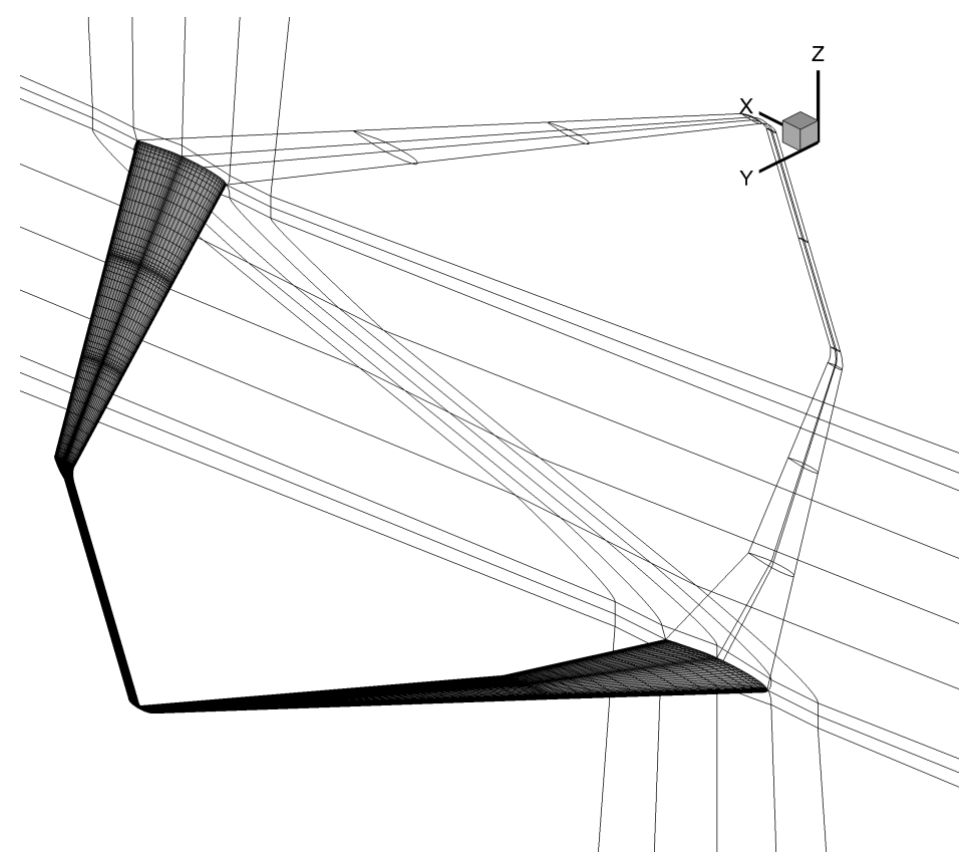

Figure 3.17: BW100: Surface mesh and blocking topology.

Table 3.10: BW100: Grid information.

\begin{tabular}{lrrr}
\hline Grid & Number of Nodes & Average Off-Wall Spacing & Average $y^{+}$ \\
\hline L0 & $5.06 \times 10^{6}$ & $1.16 \times 10^{-6}$ & 0.58 \\
L1 & $9.81 \times 10^{6}$ & $8.72 \times 10^{-7}$ & 0.41 \\
L2 & $19.63 \times 10^{6}$ & $6.68 \times 10^{-7}$ & 0.30 \\
\hline
\end{tabular}

Optimization is performed on L0, while L1 and L2 are used in secondorder Richardson extrapolation for computing aerodynamic functionals. Average off-wall spacings are in units of MAC.

\subsubsection{Drag Minimization with Lift and Trim Constraints}

For the aerodynamic design optimization of the BW100, the objective is to minimize drag subject to lift and trim constraints. More formally, the optimization problem can be summarized as

$$
\begin{aligned}
\operatorname{minimize} & C_{D}, \\
\text { w.r.t. } & \mathbf{v} \\
\text { s.t. } & L=W, \quad C_{M}=0 \\
& \mathcal{C}_{\text {egeo }}=0, \quad \mathcal{C}_{\text {igeo }} \geq 0,
\end{aligned}
$$

where $\mathbf{v}=\left[\alpha, x_{\mathrm{CG}}, z_{\mathrm{CG}}, \mathbf{v}_{\text {geo }}^{\mathrm{T}}\right]^{\mathrm{T}}$ is the vector of design variables, and $\mathcal{C}_{\text {egeo }}$ and $\mathcal{C}_{\text {igeo }}$ are the vectors of geometric equality and inequality constraints, respectively. $\mathcal{C}_{\text {egeo }}$ includes the linear twist and taper constraints, and the volume centroid CG constraints, while $\mathcal{C}_{\mathrm{i}_{\text {geo }}}$ consists of 
Table 3.11: BW100: Design variables and geometric constraints.

\begin{tabular}{lccc}
\hline Parameter & Symbol & Lower & Upper \\
\hline & Design Variables $(794)$ & \\
\hline Angle of attack (1) & $\alpha$ & $-8^{\circ}$ & $+8^{\circ}$ \\
Twist (35) & $\theta$ & $-10^{\circ}$ & $+10^{\circ}$ \\
Taper (36) & $c_{x z}$ & $90 \%$ & $110 \%$ \\
Section shape (720) & $c_{z_{i}}$ & $50 \%$ & $150 \%$ \\
Streamwise CG (1) & $x_{\mathrm{CG}}$ & $-\infty$ & $+\infty$ \\
Vertical CG (1) & $z_{\mathrm{CG}}$ & $-\infty$ & $+\infty$ \\
& & & \\
& Geometric Constraints $(375)$ & $\infty$ \\
\hline Thickness-to-chord ratio (360) & $t / c$ & $80 \%$ & - \\
Volume (1) & $V$ & $1.25 V_{\text {fuel }}$ & - \\
Linear twist (6) & - & - & $\bar{x}_{V}$ \\
Linear taper (6) & - & - & $0.12 \bar{z}_{V}+0.88 \bar{z}_{\mathrm{AC}}$ \\
Streamwise CG (1) & $x_{\mathrm{CG}}$ & $\bar{x}_{V}$ & \\
Vertical CG (1) & $z_{\mathrm{CG}}$ & $0.12 \bar{z}_{V}+0.88 \bar{z}_{\mathrm{AC}}$ & \\
\hline Boug & &
\end{tabular}

Bounds given as deviations and percentages are with respect to the initial geometry.

the minimum thickness-to-chord ratio and minimum wing volume constraints, as provided in Table 3.11. A nominal cruise mission is considered at a Mach number of 0.78 and an altitude of $36,000 \mathrm{ft}$, which corresponds to a Reynolds number of $14.5 \times 10^{6}$, based on an MAC of $8.05 \mathrm{ft}$. The maximum weight at cruise is equal to that of the CTW100, i.e. 91,400 lb, as discussed in Section 2.6.

In total, 151 design iterations were completed on 288 processors. From Figure 3.18, it can be seen that Feasibility has been satisfied to an absolute tolerance of $10^{-6}$ and Optimality has been reduced by two orders of magnitude. With Feasibility met, the Merit function represents the objective function, or the drag coefficient, which converged to a value of 154 counts on the optimization grid.

The optimized geometry is characterized by an angle of attack of 0.35 degrees and is a minimum volume design with a planform area of $1,203 \mathrm{ft}^{2}$ and a wetted area of 1,290 $\mathrm{ft}^{2}$. Compared to the main wing of the CTW100, the optimized BW100 has 21\% more planform area, thus satisfying takeoff requirements. Meanwhile, the optimized BW100 has $2 \%$ less wetted area than the total wetted area of the CTW100. Geometrically, this was made possible by the significantly lower wing volume of the BW100. The relative planform area between the fore and aft wings is $S_{\mathrm{f}} /\left(S_{\mathrm{f}}+S_{\mathrm{a}}\right)=0.438$. For reference, the height-to-span and stagger-to-span ratio design variables, which were not permitted to vary, have values of 0.26 and 0.77 , respectively. The CG is located $45.55 \mathrm{ft}$ and $2.94 \mathrm{ft}$ from the leading edge of the fore wing root along the $x$ and $z$-axes, respectively. 


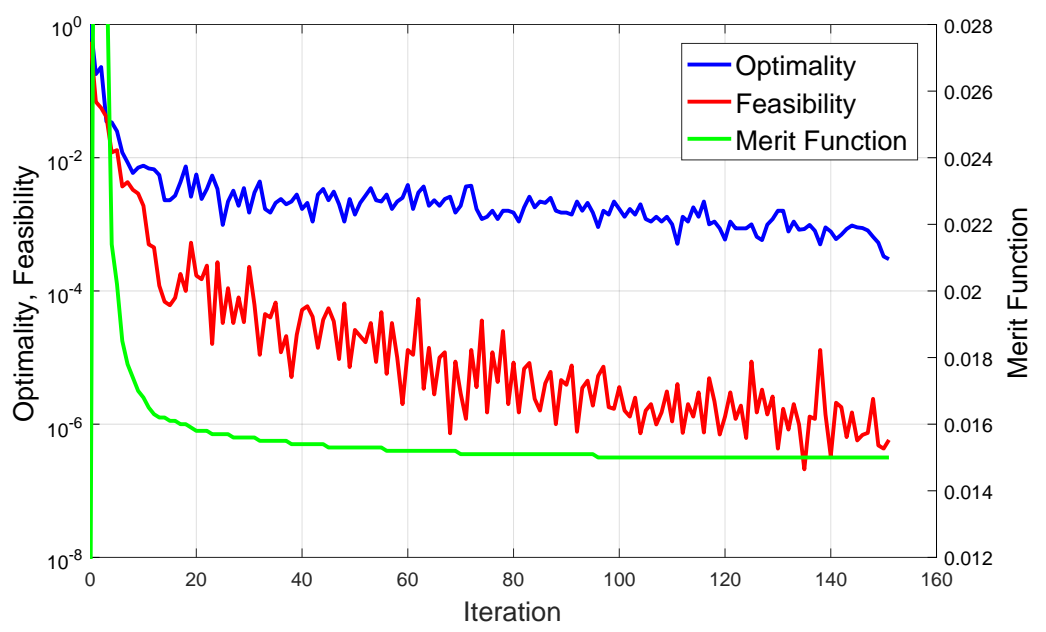

Figure 3.18: BW100: Optimization history. The Merit function represents $C_{D}$.

Figure 3.19 illustrates the pressure coefficient contours over the lower and upper surfaces of the optimized design. The smooth pressure variations indicate that wave drag has been eliminated or largely reduced. As with the BWE-2 optimization, the CG is near the center of the wing system, with a slight bias towards the aft wing, as described by the relative planform area.

Figures 3.20, 3.21, and 3.22 show the pressure coefficient distributions and section shapes of the initial and optimized designs at various spanwise and height locations for the fore and aft wings, and the vertical tip fin, respectively. These are taken at the wing root (bottom), at $20 \%, 40 \%, 60 \%$, and $80 \%$ of the semispan (height), and at the wing tip (top) of the fore and aft wings (vertical tip fin). Here, it can be seen that the optimizer has designed supercritical cambered airfoils that are well-suited for transonic flow conditions. The pressure coefficient distributions indicate that the airfoil designs are shock-free, while also maintaining gradual pressure recoveries.

The airfoil sections are also distinct at a number of spanwise stations of the fore and aft wings, which result from their adaptation to the induced flow curvature. Most apparent are the root sections of the fore and aft wings, which appear to be influenced by induced camber and angle of attack [59, 22, 93]. A close inspection of the surface pressure contours suggests that the downward bulge at the midchord of the fore wing and the upward bulge at the midchord of the aft wing have the purpose of preventing flow separation, unique to the flow interaction between each lifting surface.

As found by Gagnon and Zingg [68], the fore and aft wings are characterized by wash out (where the incidence angle decreases from the root to the tip) and wash in (where the incidence angle increases from the root to the tip), respectively. Furthermore, the force vector changes gradually from inboard to outboard from the bottom to the top of the vertical tip fin. Collectively, this forms the signature closed-loop circulation pattern shown in Figure 3.23. 


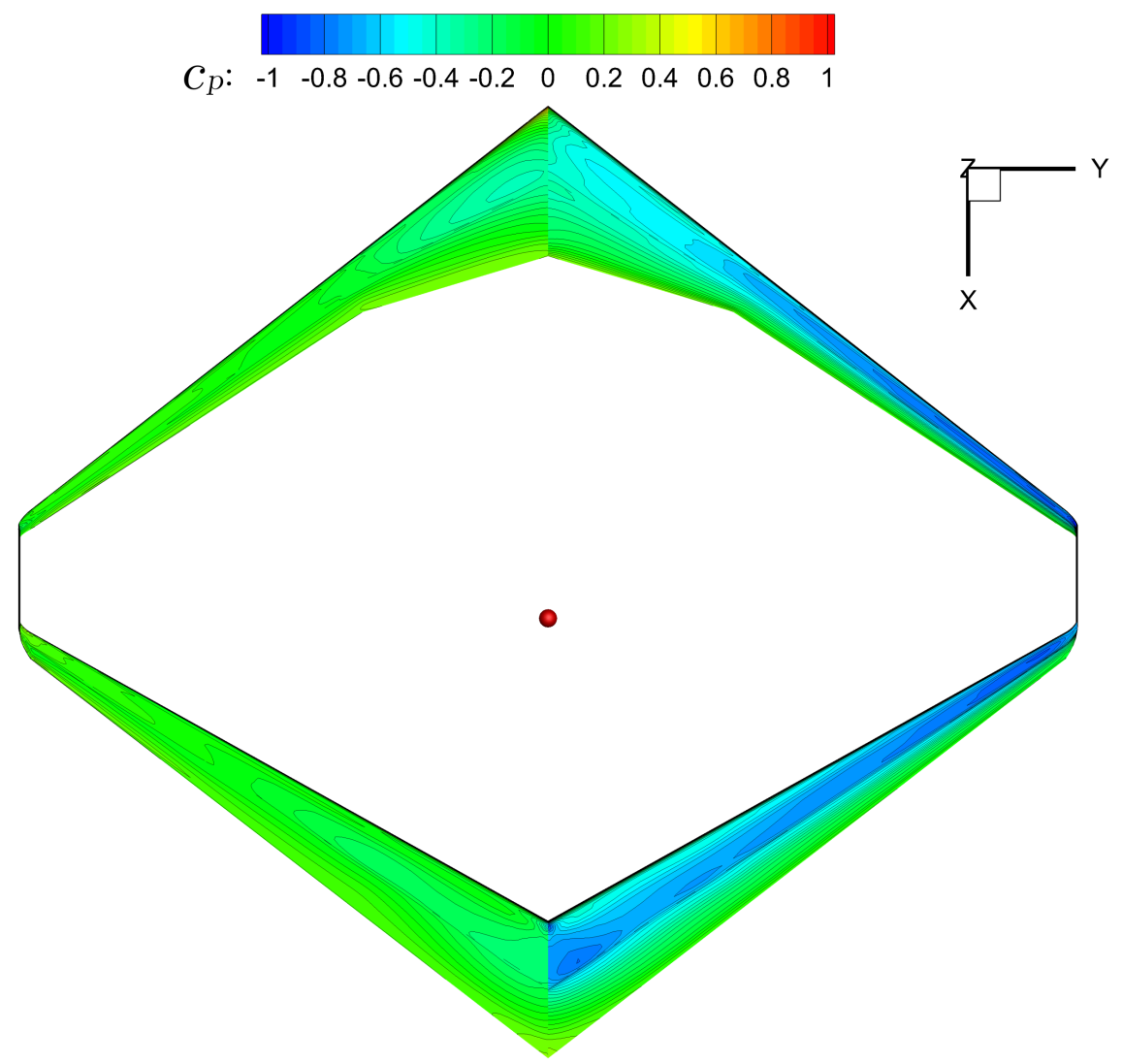

Figure 3.19: BW100: Surface pressure coefficient contours over the lower (left) and upper (right) surfaces of the optimized wing geometry. The red sphere represents the CG.

Plane cuts of the normalized $z$-component of momentum are plotted one MAC length downstream of the optimized wing geometry in Figure 3.24. Here, it is clear that the optimized box-wing configuration produces an organized trailing-wake vortex structure that maximizes the separation between the high and low pressure regions below and above the wing system, respectively, thus lowering the vertical components of momentum. Such a feature is indicative of a minimum induced drag design.

From the grid convergence study, the drag coefficient of the wing design was found to be 116 counts, which corresponds to a lift-to-drag ratio of 31.72 . When accounting for the drag contributions from the fuselage $\left(C_{D_{\mathrm{F}}}=0.0083\right)$ and the vertical stabilizer $\left(C_{D_{\mathrm{V}}}=0.0008\right)$, the drag coefficient of the regional aircraft has a value of 207 counts. For the same lift, the lift-todrag ratio of the box-wing aircraft configuration is 17.83. Based on Equation 1.1, the BW100 burns $7.61 \%$ less fuel than the CTW100 at cruise. An overview of the grid convergence study is given in Table 3.12, while a summary of the optimization results is provided in Table 3.13. 


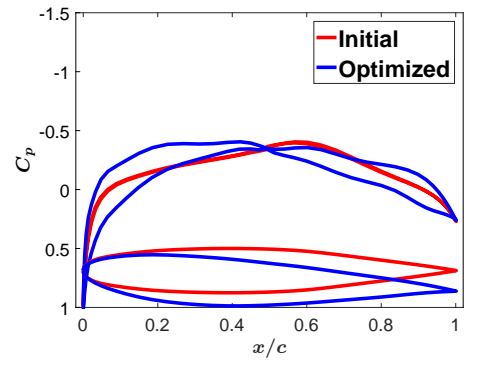

(a) Wing root

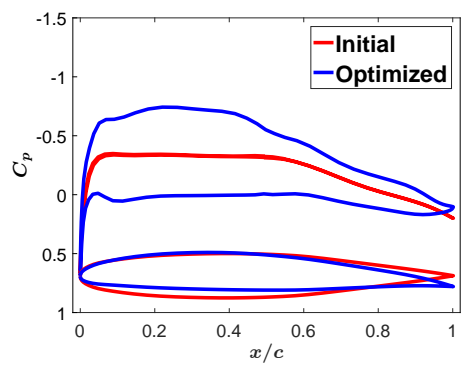

(d) $60 \% \mathrm{span}$

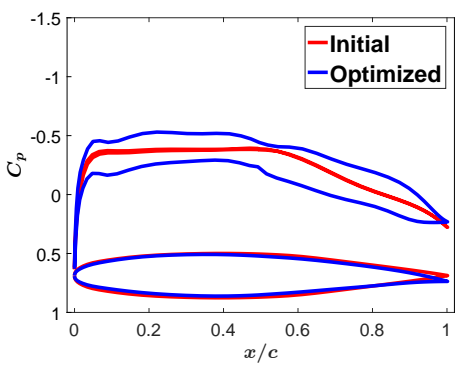

(b) $20 \% \mathrm{span}$

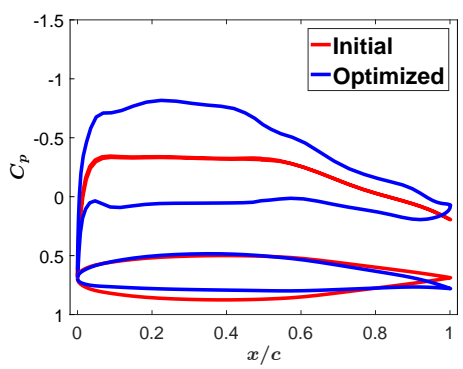

(e) $80 \%$ span

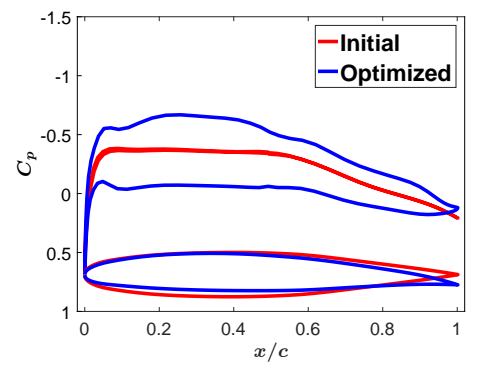

(c) $40 \%$ span

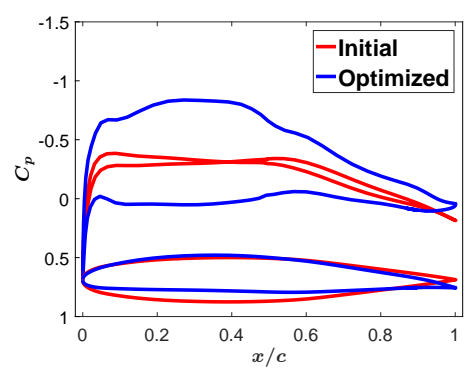

(f) Wing tip

Figure 3.20: BW100: Pressure coefficient distributions and airfoil profiles for the fore wing of the box-wing aircraft configuration.

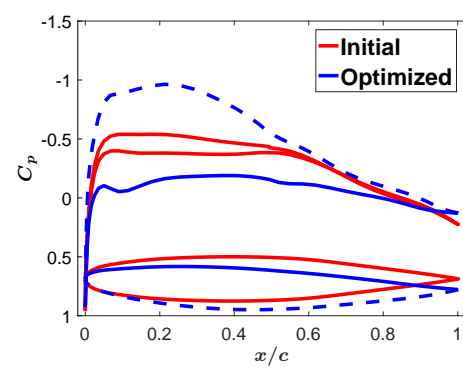

(a) Bottom

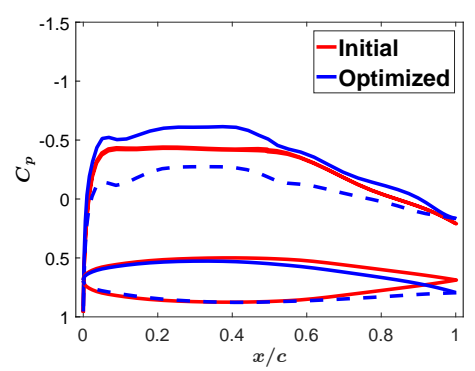

(d) $60 \%$ height

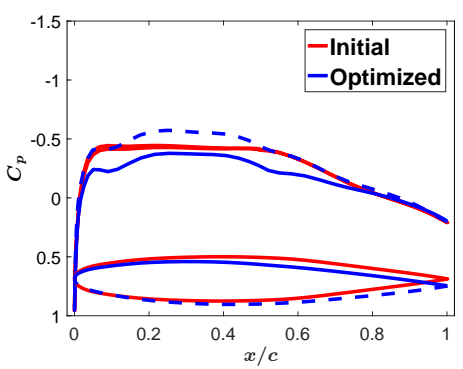

(b) $20 \%$ height

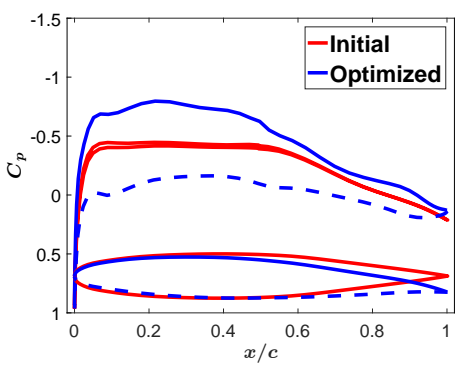

(e) $80 \%$ height

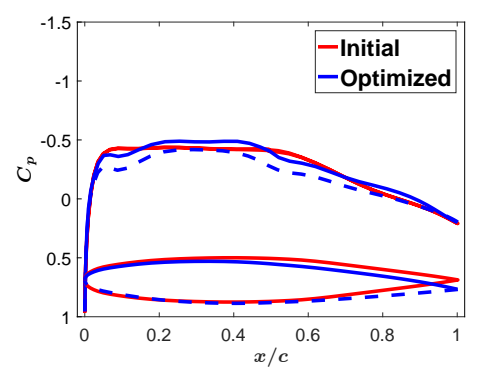

(c) $40 \%$ height

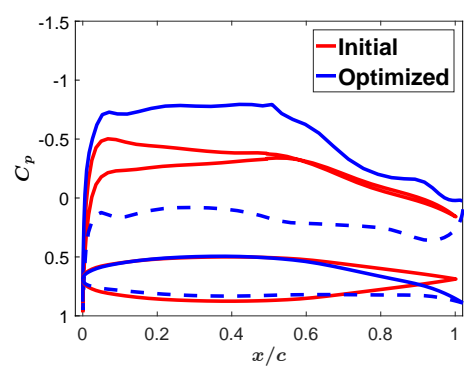

(f) Top

Figure 3.21: BW100: Pressure coefficient distributions and airfoil profiles for the vertical tip fin of the box-wing aircraft configuration. For the optimized geometry, solid lines represent outboard surfaces, and dashed lines represent inboard surfaces. 


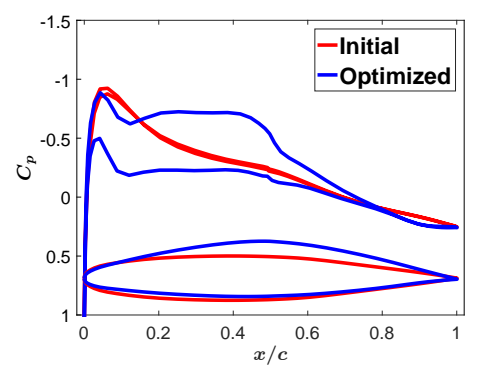

(a) Wing root

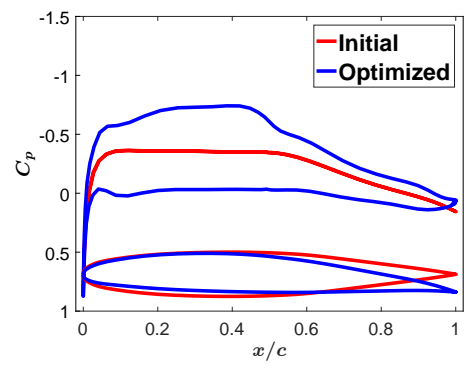

(d) $60 \% \mathrm{span}$

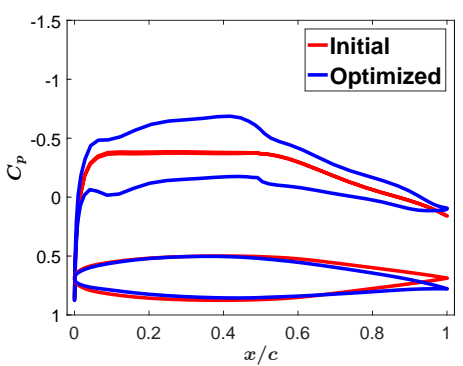

(b) $20 \%$ span

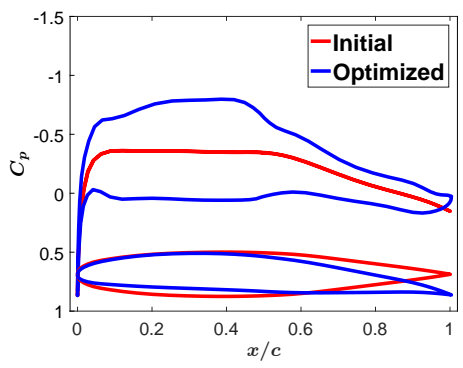

(e) $80 \%$ span

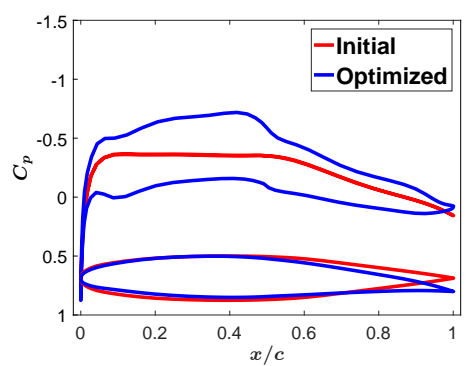

(c) $40 \%$ span

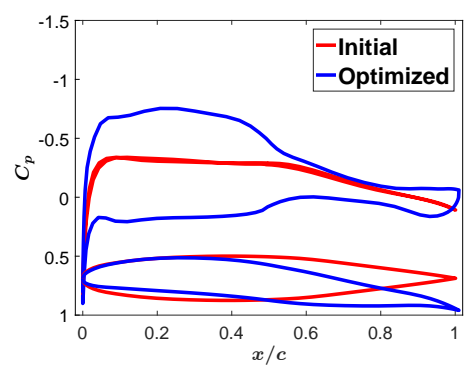

(f) Wing tip

Figure 3.22: BW100: Pressure coefficient distributions and airfoil profiles for the aft wing of the box-wing aircraft configuration.
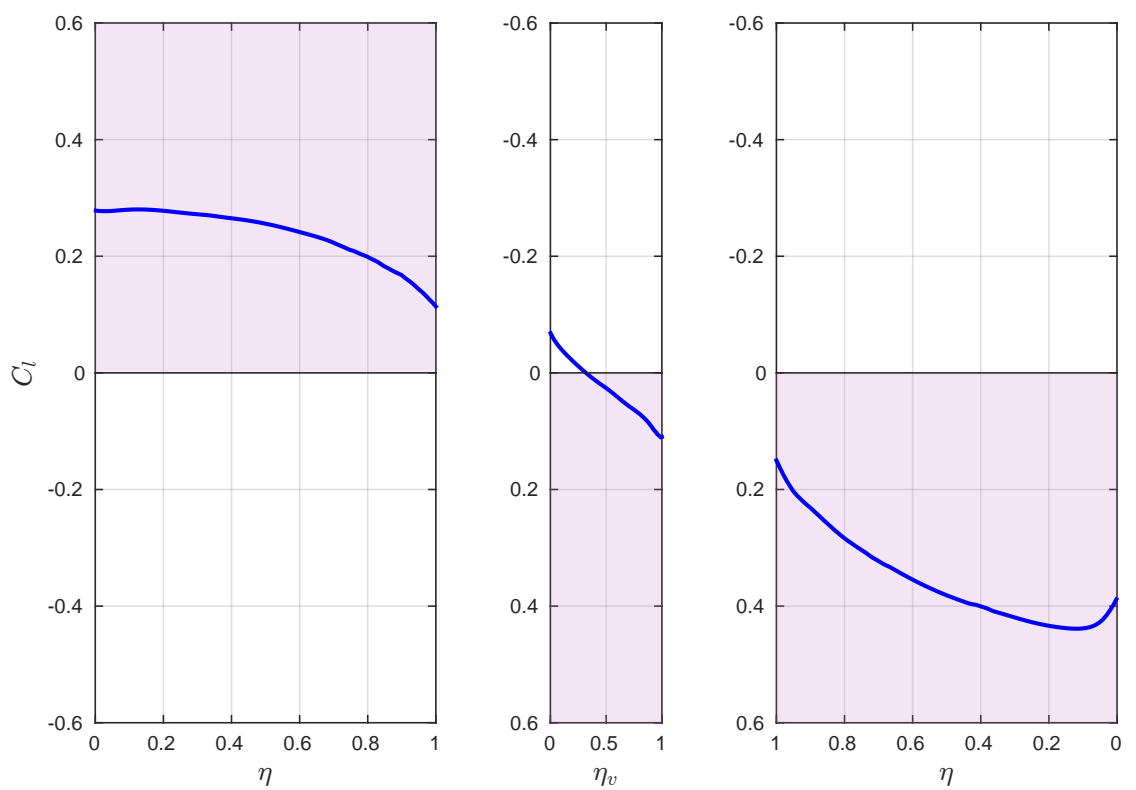

Figure 3.23: BW100: Force distributions over the fore wing (left), the vertical tip fin (middle), and the aft wing (right). 


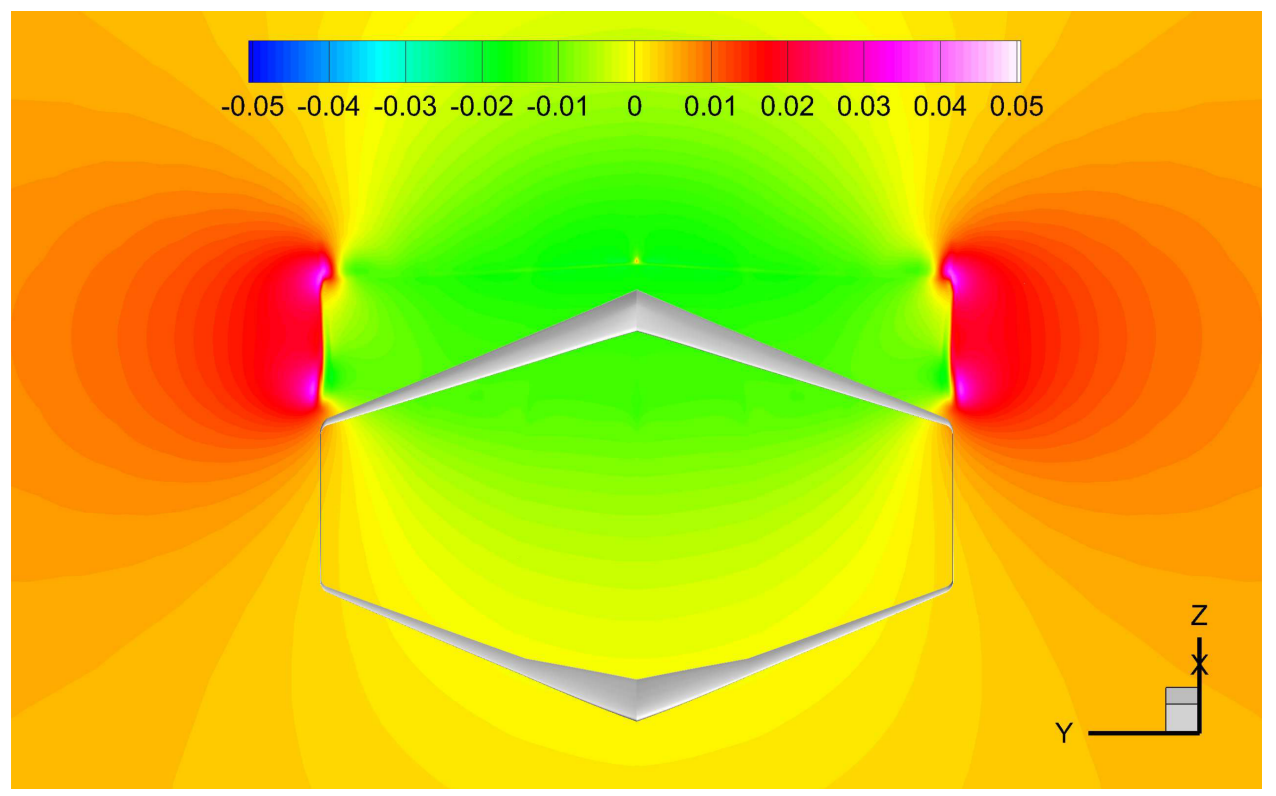

Figure 3.24: BW100: Plane cut of the normalized $z$-component of momentum located one MAC length downstream of the optimized wing geometry.

Table 3.12: BW100: Grid convergence study.

\begin{tabular}{rrrrrrrrr}
\hline Grid & Number of Nodes & Average $y^{+}$ & $C_{L}$ & $C_{D_{\mathrm{W}}}$ & $C_{D_{f_{\mathrm{W}}}}$ & $C_{D_{p_{\mathrm{W}}}}$ & $D_{f_{\mathrm{W}}} / D_{p_{\mathrm{W}}}$ & $L / D_{\mathrm{W}}$ \\
\hline L0 & $5.06 \times 10^{6}$ & 0.58 & 0.375 & 0.0154 & 0.0072 & 0.0082 & 0.883 & 24.35 \\
L1 & $9.81 \times 10^{6}$ & 0.41 & 0.377 & 0.0141 & 0.0066 & 0.0075 & 0.897 & 26.77 \\
L2 & $19.63 \times 10^{6}$ & 0.30 & 0.378 & 0.0132 & 0.0063 & 0.0069 & 0.904 & 28.60 \\
\hline
\end{tabular}

$\mathrm{W}$ represents the wing geometry only.

Table 3.13: BW100: Optimization results.

\begin{tabular}{rrrrrrrrrrrr}
\hline $\begin{array}{r}\text { Weight* } \\
{[\mathrm{lb}]}\end{array}$ & $\begin{array}{r}S \\
{\left[\mathrm{ft}^{2}\right]}\end{array}$ & $\begin{array}{r}S_{\text {wet }} \\
{\left[\mathrm{ft}^{2}\right]}\end{array}$ & $\begin{array}{r}C_{L} \\
{[-]}\end{array}$ & $\begin{array}{r}C_{D_{\mathrm{W}}} \\
{[-]}\end{array}$ & $\begin{array}{r}L / D_{\mathrm{W}} \\
{[-]}\end{array}$ & $\begin{array}{r}C_{D} \\
{[-]}\end{array}$ & $\begin{array}{r}C_{D_{f}} \\
{[-]}\end{array}$ & $\begin{array}{r}C_{D_{p}} \\
{[-]}\end{array}$ & $\begin{array}{r}L / D \\
{[-]}\end{array}$ & $\begin{array}{r}D / q_{\infty} \\
{\left[\mathrm{ft}^{2}\right]}\end{array}$ & $\begin{array}{r}\text { Drag } \\
{[\mathrm{lb}]}\end{array}$ \\
\hline 91,400 & 1,203 & 1,290 & 0.369 & 0.0116 & 31.72 & 0.0207 & 0.0146 & 0.0061 & 17.83 & 25.35 & 5,126 \\
\hline
\end{tabular}

* indicates the start of the nominal mission cruise and $\mathrm{W}$ represents the wing geometry only.

\subsubsection{Weight Sensitivity Studies}

One of the assumptions made during the aerodynamic design optimization of the box-wing configuration was that of equal structural weight or OEW between the BW100 and the CTW100 regional aircraft. This assumption was necessitated by the inapplicability of the empirical weight models to unconventional aircraft configurations such as the box wing. Furthermore, the incorrect use of such methods would make less clear whether improvements in performance offered by the BW100 over the CTW100 were attributed to the aerodynamic advantages of the former or to the limitations of the approximation.

This decision was also supported by the work of Andrews and Perez [67] and Canto et 
al. [61] who demonstrated that the wing weight to MTOW ratio of a box wing can be similar to that of an equivalent conventional baseline. In particular, Andrews and Perez applied a structural weight model to a box-wing aircraft configuration sized for short-range transport, and determined that the wing weight of the box wing was $5.7 \%$ less than that of the cantileverwing baseline, which translated to a $2.2 \%$ reduction in MTOW. From the work of Canto et al., it was found that a box-wing aircraft configuration of aluminum construction, when including twin vertical stabilizers, had a wing weight to MTOW ratio ranging from $15.8 \%$ to $17.5 \%$, depending on the size and mission of the aircraft. Nonetheless, a weight sensitivity study can be useful for providing insight as to how the assumption of equal weight can affect the overall predicted performance of the box-wing aircraft configuration. More specifically, it can aid in understanding the effect that changes in weight have on aerodynamic performance, which can be due to the greater structural efficiency offered by the braced wing system, or the greater structural support required by a twin vertical stabilizer design. To this end, the aerodynamic design optimization is repeated twice: once with a $5 \%$ increase in the maximum weight at cruise, and once with a $5 \%$ decrease in the maximum weight at cruise.

Figure 3.25 illustrates the closed-loop circulations produced for each lift target. Here it can be seen that the fore and aft wings adjust the amount of lift in a way that is proportional to the change in target lift. Furthermore, little to no change is observed from the side-force distribution over the vertical tip fin.

The results of the weight sensitivity study are summarized in Table 3.14 alongside the results of the CTW100 and the BW100 aerodynamic design optimizations. If the weight of the box-wing regional aircraft was to increase by $5 \%$, the fuel burn advantage would decrease to $6.32 \%$ ( $\Delta \approx-1 \%$ relative to the optimized BW100) over the CTW100. On the other hand, if the weight of the box-wing regional aircraft was to decrease by $5 \%$, the fuel burn advantage would increase to $9.34 \%$ ( $\Delta \approx+1.5 \%$ relative to the optimized BW100) over the CTW100. In both instances, the deviation in fuel-burn is modest, despite the sizeable change in maximum weight at cruise. 

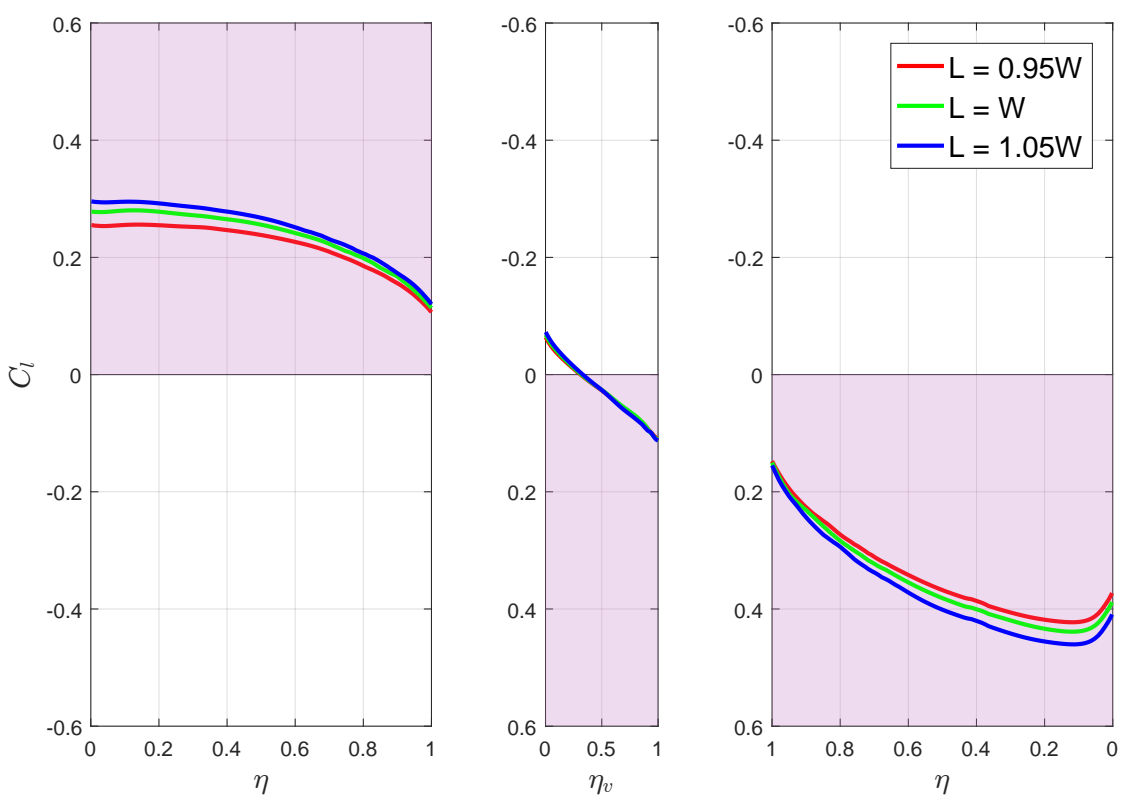

Figure 3.25: Weight Sensitivity: Force distributions over the fore wing (left), the vertical tip fin (middle), and the aft wing (right).

Table 3.14: Weight Sensitivity: Optimization results.

\begin{tabular}{|c|c|c|c|c|c|c|c|c|c|c|}
\hline Design & $\begin{array}{r}\text { Weight* } \\
{[\mathrm{lb}]}\end{array}$ & $\begin{array}{r}S \\
{\left[\mathrm{ft}^{2}\right]}\end{array}$ & $\begin{array}{l}S_{\text {wet }} \\
{\left[\mathrm{ft}^{2}\right]}\end{array}$ & $\begin{array}{c}C_{L} \\
{[-]}\end{array}$ & $\begin{array}{r}C_{D} \\
{[-]}\end{array}$ & $\begin{array}{r}D_{f} / D \\
{[-]}\end{array}$ & $\begin{array}{r}L / D \\
{[-]}\end{array}$ & $\begin{array}{r}D / q_{\infty} \\
{\left[\mathrm{ft}^{2}\right]}\end{array}$ & $\begin{array}{r}\text { Drag } \\
{[\mathrm{lb}]}\end{array}$ & $\begin{array}{r}\Delta_{\text {fuel }} \\
{[\%]}\end{array}$ \\
\hline $\mathrm{C}^{\prime}$ & 91,400 & 997 & 318 & 0.369 & 0274 & 0.64 & 16.50 & 27.40 & 5,541 & - \\
\hline$-\mathrm{m} 5$ & 30 & ,202 & 1,290 & 0.350 & 03 & 0.72 & 17.24 & 24.91 & 5,037 & -9.34 \\
\hline BV & 91,400 & 1,203 & 1,290 & 0.369 & 0.0207 & 0.71 & 17.83 & 25.35 & 5,126 & -7.61 \\
\hline BW100-p5 & 95,970 & 1,208 & 1,295 & 0.386 & 0.0212 & 0.69 & 18.21 & 26.06 & 5,270 & -6.32 \\
\hline
\end{tabular}

* indicates the start of the nominal mission cruise. $\Delta_{\text {fuel }}$ is the cruise fuel-burn relative to the optimized CTW100. 


\section{Chapter 4}

\section{Euler- and RANS-Based Aerodynamic Shape Optimization Discussion}

\subsection{Problem Setup Comparison with Gagnon and Zingg}

In the studies done by Gagnon and Zingg [68, 69], aerodynamic shape optimization based on the Euler equations was used in the design of box-wing regional aircraft, with emphasis on the importance of modeling nonlinear aerodynamics. Although the present study focuses more on aerodynamic design in the context of practical applications, namely, with more realistic viscous flow conditions, a comparison between Euler- and RANS-based optimization results can aid in understanding the advantages and disadvantages of the box-wing aircraft configuration by distinguishing inviscid and viscous aerodynamic phenomena. Such a comparison is made possible by similarities between the size and design mission of the aircraft considered by Gagnon and Zingg, and that of the aircraft considered in the present study, as well as similarities between the optimization problem formulations.

Although the problem setup of Gagnon and Zingg is not exactly the same as that of the present study, their work considers a box-wing regional aircraft of similar size and design mission. In particular, each aircraft is based on the Bombardier CRJ-1000, with a nominal cruise mission of transporting 100 passengers and 3 crew over a distance of $500 \mathrm{nmi}$ at a Mach number of 0.78 and an altitude of 35,000 ft. Recall that in the present study, the CTW100 and the BW100 are based on the Embraer E190, with a nominal cruise mission of transporting 100 passengers and 5 crew over a distance of $500 \mathrm{nmi}$ at a Mach number of 0.78 and an altitude of $36,000 \mathrm{ft}$. As with the present study, only the primary lifting surfaces are modeled, and include the fuselage-embedded regions. In the work of Gagnon and Zingg, the cantilever-wing and box-wing aircraft configurations are of equal span, at $85.96 \mathrm{ft}$, whereas the CTW100 and the BW100 have spans of $94 \mathrm{ft}$. The box-wing configurations of Gagnon and Zingg have leading- 
edge sweep angles of 40 degrees and -23 degrees on the fore and aft wings, respectively, while for the optimized BW100 of the present study, these values correspond to 38 degrees and -29.1 degrees, respectively. The CTW100 and the BW100 are of the same weight, while in the work of Gagnon and Zingg, the box wing is $2.68 \%$ heavier than the equivalent conventional baseline at cruise. Lastly, the regional aircraft of Gagnon and Zingg are of similar wetted area, as with the CTW100 and the BW100.

Like the present study, Gagnon and Zingg use free-form and axial deformation geometry control, with a similar number of FFD-volume cross-sections per unit span. In addition, each FFD-volume cross-section has the same number of FFD control points. For the studies involving drag minimization with lift and trim constraints, Gagnon and Zingg use angle of attack, twist, and section shape design variables, with planform degrees of freedom inactive. However, in Gagnon and Zingg [69], the $x$-coordinate of an axial curve control point at the midspan of the aft wing is active, allowing for nonlinear planform variations through a quadratic B-spline. Geometric constraints included minimum thickness-to-chord ratio, minimum wing volume, and linear twist. The CG was calculated using a low-fidelity weight approximation based on wetted area, and was not used as a design variable throughout the optimization, given that planform changes were not allowed.

\subsection{Results Comparison with Gagnon and Zingg}

Through aerodynamic shape optimization based on the Euler equations, Gagnon and Zingg found that a box-wing aircraft configuration with an average height-to-span ratio of 0.2 experiences $27.4 \%$ less induced drag than a similarly-sized cantilever-wing design when the objective is to minimize drag with lift and trim constraints [68]. With the addition of planform variations on the aft wing and an effective increase in the average height-to-span ratio to about 0.3 , this benefit was found to increase to $34.1 \%$. These translate to drag reductions of $10.96 \%$ and $13.64 \%$, respectively, under the assumption that $40 \%$ of the total aircraft drag is induced drag [27]. In comparison, aerodynamic shape optimization based on the RANS equations indicates that the optimized BW100, which has an average height-to-span ratio of 0.26 , experiences $7.49 \%$ less drag than the optimized CTW100. Assuming that wave drag has been largely eliminated by the section shape degrees of freedom, such a deficit can be explained by the friction drag of the optimized BW100, which comprises $71 \%$ of the total drag. This is likely a consequence of the shorter chord lengths of the fore and aft wings (recall that friction drag is inversely proportional to $\log _{10}(\mathrm{Re})^{2.58}$ for turbulent boundary layers, according to the Prandtl-Schlichting approximation [89]), as well as the overestimation of viscous drag that comes from the carry-through regions of the lifting surfaces. However, it should be noted that for the optimized wing geometry of the BW100, the friction-to-pressure drag ratio was found to be close to unity, which suggests that the planform area is aerodynamically optimal.

Now although the RANS equations predict that the BW100 experiences more viscous drag 
than the CTW100, the underlying aerodynamic advantages, which are inviscid in nature, are still preserved. This can be seen through the force distributions over the fore and aft wings, and the vertical tip fin of the optimized BW100. Recall that these correspond to wash-out and wash-in on the fore and aft wings, respectively, and a gradual change from an inboard force to an outboard force from the bottom to the top of the vertical tip fin. This forms the closed-loop circulation pattern predicted by classical lifting-line theory, which is curiously nearly identical to that which was produced by Gagnon and Zingg [68]. As such, the plane cuts of the $z$-component of momentum are also nearly identical in signature between the inviscid and viscous optimizations.

Between the Euler- and RANS-based aerodynamic shape optimization results, another major difference is in the optimized airfoil profiles. As demonstrated by Osusky et al. [47], the Euler equations tend to lead to the design of highly aft-loaded section shapes that are not of practical interest, given their susceptibility to boundary-layer separation. Although such a phenomenon does not have a large impact on the relative performance of the box wing, the omission of flow separation effects can be taken advantage of by the optimizer. Indeed, this was observed by Gagnon and Zingg, who found that the optimizer reached the lower bound of angle of attack in order to effectively increase the average height-to-span ratio from 0.2 to 0.3. Meanwhile, this was likely not possible for the BW100 optimization, given the potential vulnerability to boundary-layer separation on the lower surface associated with such a change.

Gagnon and Zingg also performed a number of trade studies where longitudinal static stability and root bending moment constraints were considered in addition to the lift and trim constraints. Although these constraints were not included in the present study, it is still important to note that the box wing of Gagnon and Zingg was more readily able to adapt to these adverse conditions than their equivalent conventional baseline, culminating in a $42.6 \%$ reduction in induced drag or a $17.04 \%$ reduction in total drag. Although these trends are based on the Euler equations, there is no particular reason to believe that these inviscid properties would not manifest themselves in aerodynamic shape optimization based on the RANS equations. 


\section{Chapter 5}

\section{Conclusions and Recommendations}

\subsection{Conclusions}

Aerodynamic shape optimization based on the RANS equations was used in the design and performance evaluation of a box-wing regional aircraft. This was done following a two-step approach in which exploratory aerodynamic shape optimization was first performed to refine the initial planform, given significant geometric freedom. The objective was to maximize the lift-todrag ratio subject to a trim constraint, which allowed the optimizer to realize the aerodynamic advantages of the box-wing configuration, while also ensuring that the relative planform area between the fore and aft wings was sized for trimmed flight. The results of the exploratory optimization indicate that an optimized closed-wing system with a height-to-span ratio of 0.26 can provide a $16.44 \%$ improvement in lift-to-drag ratio over an equivalent conventional wing-tail geometry.

The exploratory aerodynamic shape optimization also provided an opportunity for investigating important aerodynamic trends and trade-offs. For one, it was found that the leading-edge sweep of the fore and aft wings increased to their maximum bounds, namely, +10 degrees for the fore wing and -10 degrees for the aft wing, translating to +38 degrees of leading-edge sweep and -38 degrees of trailing-edge sweep for the fore and the aft wings, respectively. The heightto-span ratio also increased to its maximum bound, despite an increase in wetted area from the vertical tip fin. Although the bounds on this degree of freedom were small, such a behavior indicates that the lower induced drag predicted by linear aerodynamic theory, at least within this local region of the design space, outweighed the increase in viscous drag.

Meanwhile, the stagger-to-span ratio, which was given significantly more freedom than the height-to-span ratio, settled on an optimal value. In this case, the leading-edge sweep angles of the fore and aft wings were leveraged to enable a reduction in mutual induction between the fore and aft wings, especially near the root, without an increase in wetted area from the vertical tip fin. However, once the bounds on the leading-edge sweep design variables became active, increasing the stagger-to-span ratio further was subject to diminishing returns.

The optimized planform settled on a relative planform area of $S_{\mathrm{f}} /\left(S_{\mathrm{f}}+S_{\mathrm{a}}\right)=0.461$, with the 
optimal CG centered between the fore and aft wings. The optimizer favored allocating planform area to the aft wing over the fore wing, perhaps in an attempt to benefit from the asymmetry in mutual induction. In general, the taper design variables of the fore and aft wings also settled on optimum values, while those of the vertical tip fin reached their minimum bounds. Based on an observation of the force distribution over the vertical tip fin, it was concluded that only a minimal side force was necessary to complete the closed-loop circulation pattern.

Aerodynamic shape optimization was then applied to the design of a cantilever-wing and box-wing regional aircraft, with the former serving as a performance baseline for the latter. The objective was to minimize drag subject to lift and trim constraints. Design variables included angle of attack, twist, section shape, and taper, with the CG constrained based on the volume centroid of the wing system. Other geometric constraints included minimum thickness-to-chord ratio, minimum wing volume, linear twist, and linear taper. The size and design mission of each aircraft were based on the Embraer E190 regional jet, and a nominal cruise mission was considered where 100 passengers were to be transported $500 \mathrm{nmi}$ at a Mach number of 0.78 and an altitude of $36,000 \mathrm{ft}$. The result of the box wing optimization was a minimum volume design with $21 \%$ more planform area than the primary lifting surface of the conventional baseline, and $2 \%$ less wetted area than the main wing and horizontal stabilizer of the cantilever-wing configuration combined. The relative planform area of the optimized box wing was found to be $S_{\mathrm{f}} /\left(S_{\mathrm{f}}+S_{\mathrm{a}}\right)=0.438$, which was adjusted from that of the exploratory optimization to account for a modest change in the lift coefficient. Once again, this result suggests that an optimal box-wing configuration prefers to generate more lift from the aft wing than the fore wing.

The optimized box-wing regional aircraft has a height-to-span ratio of 0.26 , which contributes to a $7.61 \%$ reduction in fuel burn at cruise over the optimized cantilever-wing design. This advantage was attributed to a minimum induced drag closed-loop circulation pattern which was nearly identical to that of Gagnon and Zingg [68, 69] who applied aerodynamic shape optimization based on the Euler equations. However, the shorter chord lengths of the box wing led to a higher friction-to-pressure drag ratio than the similarly-sized cantilever wing, which translated to a lower predicted aerodynamic performance than that of inviscid optimization.

Finally, a weight sensitivity study was performed in which the aerodynamic design optimization of the box-wing aircraft configuration was repeated with $\pm 5 \%$ changes in target lift or maximum weight at cruise. Results indicate that a 5\% decrease in cruise lift would increase the fuel-burn advantage to $9.34 \%$ over the conventional baseline aircraft, whereas a $5 \%$ increase in cruise lift would reduce the fuel-burn advantage to $6.32 \%$. These results suggest that a boxwing regional aircraft can provide a significant improvement in performance over a conventional cantilever-wing aircraft of the same class, despite uncertainties in structural weight. 


\subsection{Recommendations}

Fuselage As a next step in studying the box-wing aircraft configuration via aerodynamic shape optimization, the fuselage geometry could be included to account for wing-body flow interactions and to correct for the less efficient lift provided by the fuselage. In addition, by including the fuselage, the over-prediction in viscous drag that comes from the carry-through regions of the aerodynamic surfaces can be avoided.

Weight Although available academic literature [67, 61] suggests that a box-wing aircraft configuration can be designed with an OEW similar to an equivalent conventional baseline, the use of a wing weight model could prove useful for understanding the sensitivity of performance with respect to the structural characteristics of a given closed wing-system.

Twin Vertical Stabilizers Frediani [58] suggested the viability of a twin vertical stabilizer design as a solution to the aeroelastic instabilities uncovered by Lange et al. [60]. However, although a preliminary structural analysis [61] indicates that such a design can be obtained without a significant penalty in structural weight, an investigation into the aerodynamic performance of such a configuration must be explored. Indeed, a twin vertical stabilizer design may be prone to shock formation, and their elimination through high-fidelity aerodynamic shape optimization remains to be seen.

Multi-Point Optimization In practice, aircraft are designed for a range of operating conditions. To address this, multi-point optimization can be performed to obtain a robust box wing design that remains aerodynamically efficient at off-design conditions.

Aerostructural Optimization The braced wing structure of the box-wing aircraft configuration can allow it to benefit from having a lower weight, but at the same time, the implementation of a twin vertical stabilizer design could diminish these gains. As such, aerostructural optimization should be performed to understand the effect of structures and therefore, weight, on performance. Aerostructural optimization would also enable trade-offs between aerodynamics and structures, allowing for a more practical sizing of the vertical tip fin as well as wing sweep, height, and stagger. Aeroelastic tailoring could also be included to provide better aerodynamic performance over a range of operating conditions.

Flutter Ultimately, the feasibility of the box-wing aircraft configuration will depend on whether a fuel-efficient design can be derived while remaining aeroelastically stable, as suggested by Lange et al. [60]. The implementation of a flutter model would be invaluable in determining if such a design exists. 
Snap-Buckling Recent studies [94, 95] have suggested that the unique nonlinear structural response of the box-wing aircraft configuration requires a specialized treatment for buckling. Indeed, these studies demonstrate the unreliability of linear buckling theory, which often leads to an overstimation of the critical load point. As such, snap-buckling criteria should be included in aerostructural optimization to maximize the aerodynamic and structural potential of a given box-wing design.

Multimodality In general, design spaces in three-dimensional aerodynamic shape optimization are multimodal. For this reason, a multi-start method [82] may prove useful, which would begin the gradient-based optimization from a range of initial designs, thus allowing for a more thorough exploration of the design space. However, given that the problems considered in this work are, for the most part, largely constrained, the design space may not be multimodal. 


\section{References}

[1] International Civil Aviation Organization, "Annual Report of the Council 2014," 2014.

[2] Intergovernmental Panel on Climate Change, "Climate Change 2014: Synthesis Report," 2014 .

[3] Lee, D. S., Pitari, G., Grewe, V., Penner, J. E., Petzold, A., Prather, M. J., Schumann, U., Bais, A., Bernsten, T., Iachetti, D., Lim, L. L., and Sausen, R., "Transport Impacts on Atmosphere and Climate: Aviation," Atmospheric Environment, Vol. 44, 2010, pp. 46784734.

[4] Skowron, A., Lee, D. S., and De León, R. R., "The Assessment of the Impact of Aviation $\mathrm{NO}_{x}$ on Ozone and other Radiative Forcing Responses - The Importance of Representing Cruise Altitudes Accurately," Atmospheric Environment, Vol. 74, 2013, pp. 159-168.

[5] International Air Transport Association, "IATA Annual Review 2016," 2016.

[6] Green Aviation Research and Development Network, "Annual Report 2015," 2015.

[7] Sustainable Aviation, "Progress Report 2015," 2015.

[8] NASA, "Overview of NASAs Environmentally Responsible Aviation Project," 48th AIAA Aerospace Sciences Meeting, Orlando, Florida, January 2010.

[9] Advisory Council for Aviation Research and Innovation in Europe, "The Strategic Research and Innovation Agenda for Aviation," 2012.

[10] Pratt and Whitney Canada, "The PurePower Geared Turbofan," 2016.

[11] Stuart, A. R., "The Unducted Fan Engine," 21st Joint Propulsion Conference, Monterey, California, July 1985, AIAA 1985-1190.

[12] Plas, A. P., Sargeant, M. A., Madani, V., Crichton, D., Greitzer, E. M., Hynes, T. P., and Hall, C. A., "Performance of a Boundary Layer Ingesting (BLI) Propulsion System," 45th AIAA Aerospace Sciences Meeting and Exhibit, Reno, Nevada, January 2007, AIAA 2007-450. 
[13] Blumenthal, B. T., Elmiligui, A. A., Geiselhart, K. A., Campbell, R. L., Maughmer, M. D., and Schmitz, S., "Computational Investigation of a Boundary-Layer Ingesting Propulsion System for the Common Research Model," 46th AIAA Fluid Dynamics Conference, Washington, D.C., June 2016, AIAA 2016-3812.

[14] Gohardani, A. S., Doulgeris, G., and Singh, R., "Challenges of Future Aircraft Propulsion: A Review of Distributed Propulsion Technology and its Potential Application for the All Electric Commercial Aircraft," Progress in Aerospace Sciences, Vol. 47, 2011, pp. 369-391.

[15] The Boeing Company, "Boeing 787 from the Ground Up," 2006, Iss. 24, Q4.

[16] Airbus SAS, "The A350 XWB," 2016.

[17] Liebeck, R., "Design of the Blended Wing Body Subsonic Transport," Journal of Aircraft, Vol. 41, No. 1, 2014, pp. 10-25.

[18] Reist, T. A. and Zingg, D. W., "High-Fidelity Aerodynamic Shape Optimization of a Lifting-Fuselage Concept for Regional Aircraft," Journal of Aircraft, 2016, (In press).

[19] Drela, M., "Development of the D8 Transport Configuration," 29th AIAA Applied Aerodynamics Conference, Honolulu, Hawaii, June 2011, AIAA 2011-3970.

[20] Pfenninger, W., "Design Considerations of Large Subsonic Long Range Transport Airplanes with Low Drag Boundary Layer Suction," Tech. rep., Northrop Aircraft Incorporated, November 1954, NAI-54-800 (BLC-67).

[21] Miranda, L. R., "Boxplane Configuration - Conceptual Analysis and Initial Experimental Verification," Tech. rep., Lockheed-California Company, March 1972, LR-25180.

[22] Wolkovitch, J., "The Joined Wing: An Overview," Journal of Aircraft, Vol. 23, No. 3, 1986, pp. 161-178.

[23] Gur, O., Bhatia, M., Schetz, J. A., Mason, W. H., Kapania, R. K., and Mavris, D. N., "Design Optimization of a Truss-Braced-Wing Transonic Transport Aircraft," Journal of Aircraft, Vol. 47, No. 6, 2010, pp. 1907-1917.

[24] Meadows, N. A., Schetz, J. A., Kapania, R. K., Bhatia, M., and Seber, G., "Multidisciplinary Design Optimization of Medium-Range Transonic Truss-Braced Wing Transport Aircraft," Journal of Aircraft, Vol. 49, No. 6, 2012, pp. 1006-1014.

[25] Grasmeyer, J. M., Naghshineh, A., Tetrault, P. A., Grossman, B., Haftka, R. T., Kapania, R. K., Mason, W. H., and Schetz, J. A., "Multidisciplinary Design Optimization of a StrutBraced Wing Aircraft," Tech. rep., NASA Langley Research Center, January 1998, MAD 98-01-01.

[26] Prandtl, L., "Induced Drag of Multiplanes," Tech. rep., NACA, March 1924, TN-182. 
[27] Kroo, I., "Nonplanar Wing Concepts for Increased Aircraft Efficiency," Innovative Configurations and Advanced Concepts for Future Civil Transport Aircraft, edited by E. Torenbeek and H. Deconinck, von Karman Institute for Fluid Dynamics, June 2005, VKI Lecture Series.

[28] Vale, J., Afonso, F., Lau, F., and Suleman, A., "Performance Based MDO of a JoinedWing Regional Transport Aircraft," 56th AIAA/ASCE/AHS/ASC Structures, Structural Dynamics, and Materials Conference, Kissimmee, Florida, January 2015, AIAA 2015-0696.

[29] Holland, J. H., Adaptation in Natural and Artificial Systems: An Introductory Analysis with Applications to Biology, Control, and Artificial Intelligence, U Michigan Press, 1st ed., 1975 .

[30] Jin, Y., "Surrogate-Assisted Evolutionary Computation: Recent Advances and Future Challenges," Swarm and Evolutionary Computation, Vol. 1, 2011, pp. 61-70.

[31] Nocedal, J. and Wright, S. J., Numerical Optimization, Springer, 2nd ed., 2006.

[32] Gill, P. E., Murray, W., and Saunders, M. A., "SNOPT: An SQP Algorithm for LargeScale Constrained Optimization," SIAM Journal on Optimization, Vol. 14, No. 4, 2002, pp. 979-1006.

[33] Fletcher, R. and Leyffer, S., "Nonlinear Programming Without a Penalty Function," Mathematical Programming, Vol. 91, No. 2, 2002, pp. 239-269.

[34] Hicks, R. M. and Henne, P. A., "Wing Design by Numerical Optimization," Journal of Aircraft, Vol. 15, No. 7, 1978, pp. 407-412.

[35] Pironneau, O., "On Optimum Design in Fluid Mechanics," Journal of Fluid Mechanics, Vol. 64, No. 1, 1974, pp. 97-110.

[36] Jameson, A., "Aerodynamic Design via Control Theory," Journal of Scientific Computing, Vol. 3, No. 3, 1998, pp. 223-260.

[37] Anderson, W. K. and Bonhaus, D. L., "Airfoil Design on Unstructured Grids for Turbulent Flows," AIAA Journal, Vol. 37, No. 2, 1999, pp. 185-191.

[38] Nemec, M. and Zingg, D. W., "Newton-Krylov Algorithm for Aerodynamic Design using the Navier-Stokes Equations," AIAA Journal, Vol. 40, No. 6, 2002, pp. 1146-1154.

[39] Spalart, P. R. and Allmaras, S. R., "A One-Equation Turbulence Model for Aerodynamic Flows," 30th AIAA Aerospace Sciences Meeting and Exhibit, Reno, Nevada, January 1992, AIAA 92-0439. 
[40] Driver, J. and Zingg, D. W., "Numerical Aerodynamic Optimization Incorporating Laminar-Turbulent Transition Prediction," AIAA Journal, Vol. 45, No. 8, 2007, pp. 18101818.

[41] Hicken, J. E. and Zingg, D. W., "Parallel Newton-Krylov Solver for the Euler Equations Discretized Using Simultaneous-Approximation Terms," AIAA Journal, Vol. 46, No. 11, 2008, pp. 2773-2786.

[42] Hicken, J. E. and Zingg, D. W., "Aerodynamic Optimization Algorithm with Integrated Geometry Parameterization and Mesh Movement," AIAA Journal, Vol. 48, No. 2, 2010, pp. 400-413.

[43] Hicken, J. E. and Zingg, D. W., "Induced-Drag Minimization of Nonplanar Geometries Based on the Euler Equations," AIAA Journal, Vol. 48, No. 11, 2010, pp. 2564-2575.

[44] Elliott, J. K., Aerodynamic Optimization based on the Euler and Navier-Stokes Equations using Unstructured Grids, Ph.D. thesis, Massachusetts Institute of Technology, Cambridge, Massachusetts, USA, 1998.

[45] Osusky, M. and Zingg, D. W., "Parallel Newton-Krylov-Schur Solver for the Navier-Stokes Equations Discretized Using Summation-By-Parts Operators," AIAA Journal, Vol. 51, No. 12, 2013, pp. 2833-2851.

[46] Osusky, L. and Zingg, D. W., "Application of an Efficient Newton-Krylov Algorithm for Aerodynamic Shape Optimization Based on the Reynolds-Averaged Navier-Stokes Equations," 21st AIAA Computational Fluid Dynamics Conference, San Diego, California, June 2013, AIAA 2013-2584.

[47] Osusky, L., Buckley, H. P., Reist, T. A., and Zingg, D. W., "Drag Minimization Based on the Navier-Stokes Equations Using a Newton-Krylov Approach," AIAA Journal, Vol. 53, No. 6, 2015, pp. 1555-1577.

[48] Koo, D. and Zingg, D. W., "Progress in Aerodynamic Shape Optimization Based on the Reynolds-Averaged Navier-Stokes Equations," 54th AIAA Aerospace Sciences Meeting, San Diego, California, January 2016, AIAA 2015-1292.

[49] Jameson, A., Martinelli, L., and Pierce, N. A., "Optimum Aerodynamic Design Using the Navier-Stokes Equations," Theoretical and Computational Fluid Dynamics, Vol. 10, No. 6, 1998, pp. 213-237.

[50] Jameson, A., Shankaran, S., Martinelli, L., Cliff, S., and Thomas, S., "Aerodynamic Shape Optimization of Transonic and Supersonic Aircraft Configurations," 43rd AIAA Aerospace Sciences Meeting and Exhibit, Reno, Nevada, January 2005, AIAA 2005-1013. 
[51] Brezillon, J., Brodersen, O., Dwight, R. P., Ronzheimer, R., and Wild, J., "Development and Application of a Flexible and Efficient Environment for Aerodynamic Shape Optimization," Proceedings of the ONERA-DLR Aerospace Symposium (ODAS), Toulouse, France, October 2006.

[52] Wild, J., Brezillon, J., Amoignon, O., Quest, J., Moens, F., and Quagliarella, D., "Advanced Design by Numerical Methods and Wind-Tunnel Verification Within European High-Lift Program," Journal of Aircraft, Vol. 46, No. 1, 2009, pp. 157-167.

[53] Brezillon, J. and Dwight, R. P., "Applications of a Discrete Viscous Adjoint Method for Aerodynamic Shape Optimization of 3D Configurations," CEAS Aeronautical Journal, Vol. 3, No. 1, 2012, pp. 25-34.

[54] Kenway, G. K. and Martins, J. R. R. A., "Multipoint Aerodynamic Shape Optimization Investigations of the Common Research Model," 53rd AIAA Aerospace Sciences Meeting, Kissimmee, Florida, January 2015, AIAA 2015-0264.

[55] Lyu, Z., Kenway, G. K. W., and Martins, J. R. R. A., "Aerodynamic Shape Optimization Investigations of the Common Research Model Wing Benchmark," AIAA Journal, Vol. 53, No. 4, 2015, pp. 968-985.

[56] Demasi, L., Monegato, G., Rizzo, E., Cavallaro, R., and Dipace, A., "Minimum Induced Drag Theorems for Joined Wings, Closed Systems, and Generic Biwings: Applications," Journal of Optimization Theory and Applications, Vol. 169, No. 1, 2016, pp. 236-261.

[57] Munk, M. M., "The Minimum Induced Drag of Aerofoils," Tech. rep., NACA, January 1921, TR-121.

[58] Frediani, A., "The Prandtl Wing," Innovative Configurations and Advanced Concepts for Future Civil Transport Aircraft, edited by E. Torenbeek and H. Deconinck, von Karman Institute for Fluid Dynamics, June 2005, VKI Lecture Series.

[59] Addoms, R. B. and Spaid, F. W., "Aerodynamic Design of High-Performance Biplane Wings," Journal of Aircraft, Vol. 12, No. 8, 1975, pp. 629-630.

[60] Lange, R. H., Cahill, J. F., Bradley, E. S., Eudaily, R. R., Jenness, C. M., and MacWilkinson, D. G., "Feasibility Study of the Transonic Biplane Concept for Transport Aircraft Application," Tech. rep., The Lockheed-Georgia Company, June 1974, LG74ER0077.

[61] Canto, D. D., Frediani, A., Ghiringhelli, G. L., and Terraneo, M., "The Lifting System of a PrandtlPlane, Part 1: Design and Analysis of a Light Alloy Structural Solution," Variational Analysis and Aerospace Engineering: Mathematical Challenges for Aerospace design, edited by G. Buttazzo and A. Frediani, Vol. 66, Springer US, Boston, MA, 2012, pp. 211-234. 
[62] Divoux, N. and Frediani, A., "The Lifting System of a PrandtlPlane, Part 2: Preliminary Study on Flutter Characteristics," Variational Analysis and Aerospace Engineering: Mathematical Challenges for Aerospace design, edited by G. Buttazzo and A. Frediani, Vol. 66, Springer US, Boston, MA, 2012, pp. 235-267.

[63] Frediani, A., Cipolla, V., and Rizzo, E., "The PrandtlPlane Configuration: Overview on Possible Applications to Civil Aviation," Variational Analysis and Aerospace Engineering: Mathematical Challenges for Aerospace design, edited by G. Buttazzo and A. Frediani, Vol. 66, Springer US, Boston, MA, 2012, pp. 179-210.

[64] Oliviero, F. and Frediani, A., "Conceptual Design of a Very Large PrandtlPlane Freighter," Variational Analysis and Aerospace Engineering: Mathematical Challenges for Aerospace design, edited by G. Buttazzo and A. Frediani, Vol. 66, Springer US, Boston, MA, 2012, pp. 305-321.

[65] Frediani, A., Cipolla, V., and Oliviero, F., "Design of a Prototype of Light Amphibious PrandtlPlane," 56th AIAA/ASCE/AHS/ASC Structures, Structural Dynamics, and Materials Conference, Kissimmee, Florida, January 2015, AIAA 2015-0700.

[66] Andrews, S. A. and Perez, R. E., "Parametric Study of Box-Wing Aerodynamics for Minimum Drag Under Stability and Maneuverability Constraints," 33rd AIAA Applied Aerodynamics Conference, Dallas, Texas, June 2015, AIAA 2015-3291.

[67] Andrews, S. A. and Perez, R. E., "Multidisciplinary Analysis of a Box-Wing Aircraft Designed for a Regional-Jet Mission," 16th AIAA/ISSMO Multidisciplinary Analysis and Optimization Conference, Dallas, Texas, June 2015, AIAA 2015-2793.

[68] Gagnon, H. and Zingg, D. W., "Aerodynamic Trade Study of a Box-Wing Aircraft Configuration," Journal of Aircraft, Vol. 53, No. 4, 2016, pp. 971-981.

[69] Gagnon, H. and Zingg, D. W., "Euler-Equation-Based Drag Minimization of Unconventional Aircraft," Journal of Aircraft, Vol. 53, No. 5, 2016, pp. 1361-1371.

[70] Piegl, L. and Tiller, W., The NURBS book, Springer, 2nd ed., 1997, Monographs in Visual Communication.

[71] Gagnon, H. and Zingg, D. W., "Two-Level Free-Form and Axial Deformation for Exploratory Aerodynamic Shape Optimization," AIAA Journal, Vol. 53, No. 7, 2015, pp. 2015-2026.

[72] Lazarus, F., Coquillart, S., and Jancène, P., "Axial Deformations: an Intuitive Deformation Technique," Computer-Aided Design, Vol. 26, No. 8, 1994, pp. 607-613.

[73] Meijerink, J. A. and van der Vorst, H. A., "An Iterative Solution Method for Linear Systems of Which the Coefficient Matrix is a Symmetric M-Matrix," Mathematics of Computation, Vol. 31, No. 137, 1977, pp. 148-162. 
[74] Svärd, M., Mattsson, K., and Nordström, J., "Steady-State Computations Using Summation-by-Parts Operators," Journal of Scientific Computing, Vol. 24, No. 1, 2005, pp. 79-95.

[75] Del Rey Fernández, D. C., Hicken, J. E., and Zingg, D. W., "Review of Summation-byParts Operators with Simultaneous Approximation Terms for the Numerical Solution of Partial Differential Equations," Computers \& Fluids, Vol. 95, No. 22, 2014, pp. 171-196.

[76] Jameson, A., Schmidt, W., and Turkel, E., "14th Fluid and Plasma Dynamics Conference," Numerical Solution of the Euler Equations by Finite Volume Methods Using Runge-Kutta Time-Stepping Schemes, Palo Alto, California, June 1981, AIAA-81-1259.

[77] Pulliam, T. H., "Efficient Solution Methods for the Navier-Stokes Equations," Numerical Techniques for Viscous Flow Computation in Turbomachinery Bladings, von Karman Institute for Fluid Dynamics, January 1986, VKI Lecture Series.

[78] Swanson, R. C. and Turkel, E., "On Central-Difference and Upwind Schemes," Journal of Computational Physics, Vol. 101, No. 2, 1992, pp. 292-306.

[79] Saad, Y. and Schultz, M. H., "GMRES: A Generalized Minimal Residual Algorithm for Solving Nonsymmetric Linear Systems," SIAM Journal on Scientific and Statistical Computing, Vol. 7, No. 3, 1986, pp. 856-869.

[80] Saad, Y., "A Flexible Inner-Outer Preconditioned GMRES Algorithm," SIAM Journal on Scientific and Statistical Computing, Vol. 14, No. 2, 1993, pp. 461-469.

[81] Saad, Y. and Sosonkina, M., "Distributed Schur Complement Techniques for General Sparse Linear Systems," SIAM Journal on Scientific and Statistical Computing, Vol. 21, No. 4, 1999, pp. 1337-1357.

[82] Chernukhin, O. and Zingg, D. W., "Multimodality and Global Optimization in Aerodynamic Design," AIAA Journal, Vol. 51, No. 6, 2013, pp. 1342-1354.

[83] Squire, W. and Trapp, G., "Using Complex Variables to Estimate Derivatives of Real Functions," SIAM Review, Vol. 40, No. 1, 1998, pp. 110-112.

[84] Anderson, W. K., Newman, J. C., Whitfield, D. L., and Nielsen, E. J., "Sensitivity Analysis for Navier-Stokes Equations on Unstructured Meshes Using Complex Variables," AIAA Journal, Vol. 39, No. 1, 2001, pp. 56-63.

[85] de Sturler, E., "Nested Krylov Methods Based on GCR," Journal of Computational and Applied Mathematics, Vol. 67, No. 1, 1996, pp. 15-41.

[86] Hicken, J. E. and Zingg, D. W., "A Simplified and Flexible Variant of GCROT for Solving Nonsymmetric Linear Systems," SIAM Journal of Scientific Computing, Vol. 32, No. 3, 2010, pp. 1672-1694. 
[87] Gagnon, H. and Zingg, D. W., "Geometry Generation of Complex Unconventional Aircraft with Application to High-Fidelity Aerodynamic Shape Optimization," 21st AIAA Computational Fluid Dynamics Conference, San Diego, California, June 2013, AIAA 2013-2850.

[88] Torenbeek, E., Synthesis of Subsonic Airplane Design, Delft University, 1st ed., 1976.

[89] Raymer, D. P., Aircraft Design: A Conceptual Approach, American Institute of Aeronautics and Astronautics, 5th ed., 2012.

[90] Roskam, J., Aircraft Design Part V: Component Weight Estimation, University of Kansas, 1st ed., 1989.

[91] Meier, N., "Jet Engine Specification Database," 2005.

[92] Embraer Commercial Aircraft, Embraer 190 Airport Planning Manual, 2005, Revision 10 - 18 February 2013.

[93] Kuchemann, D., "Investigation of the Lift Distribution Over the Separate Wings of a Biplane," Tech. rep., NACA, October 1938, No. 889.

[94] Demasi, L., Cavallaro, R., and Razón, A. M., "Postcritical Analysis of PrandtlPlane JoinedWing Configurations," AIAA Journal, Vol. 51, No. 1, 2013, pp. 161-177.

[95] Cavallaro, R., Demasi, L., and Passariello, A., "Nonlinear Analysis of PrandtlPlane Joined Wings: Effects of Anisotropy," AIAA Journal, Vol. 52, No. 5, 2014, pp. 964-980. 